\title{
Hipersuperfícies mínimas e completas de espaços simétricos
}

\author{
Jaime Leonardo Orjuela Chamorro
}

TESE APRESENTADA

$\mathrm{AO}$

INSTITUTO DE MATEMÁTICA E ESTATÍSTICA

DA

UNIVERSIDADE DE SÃO PAULO

PARA

OBTENÇÃO DO TÍTULO

$\mathrm{DE}$

DOUTOR EM CIÊNCIAS

\author{
Programa: Matemática \\ Orientador: Prof. Dr. Claudio Gorodski
}

Durante o desenvolvimento deste trabalho o autor recebeu auxílio financeiro da FAPESP

São Paulo, julho de 2012 



\section{Hipersuperfíces mínimas e completas de espaços simétricos}

Esta versão da tese contém as correções e alterações sugeridas pela Comissão Julgadora durante a defesa da versão original do trabalho, realizada em 02/07/2012. Uma cópia da versão original está disponível no Instituto de Matemática e Estatística da Universidade de São Paulo.

Comissão Julgadora:

- Prof. Dr. Claudio Gorodski (orientador) - IME-USP

- Prof. Dr. Marcos Martins Alexandrino da Silva - IME-USP

- Prof. Dr. Ruy Togeiro de Figueiredo Júnior - DM-UFSCar

- Prof. Dr. Francesco Mercuri - IMECC-UNICAMP

- Prof. Dr. Marcos Jardim - IMECC-UNICAMP 

Ao generoso povo brasileiro, quem acolheu-me como mais um dos seus. 

"¡Oh Adán!

¿Cuándo estuve en ti? ¿Quién te dio mi alma y mi pecho quién te concedio el derecho de que pecaras por mi?" Rafael Pombo (1833 - 1912)

Linha curva

O caminho mias agradável entre dois pontos.

Linha reta

Linha sem imaginação.

Mario Quintana (1906 - 1994) 

Agradecimentos

Gostaria de agradecer em primeiro lugar à minha família pelo apoio incondicional. Em segundo, ao professor Claudio Gorodski pela paciência e estímulo. Em terceiro a todos os meus amigos pelos gratos momentos que me brindaram ao longo deste período. E finalmente, aos companheiros e amigos do futebol pelas vivências dentro do campo e da quadra. 


\section{Hipersuperfíces mínimas e completas de espaços simétricos}

No presente trabalho construímos novos exemplos de hipersuperfícies mínimas, completas e $H$-equivariantes de espaços simétricos. Para tal, usamos o método da geometria diferencial equivariante [HL71]. Dividimos nosso estudo em duas partes, a saber, espaços simétricos $G / K$ de tipo não compacto e compacto. No primeiro caso são estudadas ações polares de subgrupos $H$ adaptados à decomposição de Iwasawa $G=K A N$. No segundo caso usamos a classificação dos subgrupos $H$ de $\operatorname{Spin}(9)$ que atuam com cohomogeneidade dois sobre $\mathbf{P}_{\mathbf{O}}^{2}=F_{4} / \operatorname{Spin}(9)[\mathrm{PT} 99]$.

Palavras-chave: Hipersuperfícies mínimas, espaços simétricos, geometria diferencial equivariante, ações polares. 


\section{Complete minimal hypersurfaces in symmetric spaces}

In the present work we construct new examples of complete minimal $H$-equivariant hypersurfaces of symmetric spaces $G / K$. For that, we use the equivariant differential geometry method [HL71]. We divide our research in two parts, namely, symmetric spaces of noncompact and compact type. In the first case we study polar actions of subgroups $H$ adapted to the Iwasawa decomposition $G=K A N$. In the second case we use the classification of the subgroups $H$ of $\operatorname{Spin}(9)$ which act with cohomogeneity two on $\mathbf{P}_{\mathbf{O}}^{2}=F_{4} / \operatorname{Spin}(9)$ [PT99].

Keywords: Minimal hypersurfaces, symmetric spaces, equivariant differential geometry, polar actions. 
1 Geometria diferencial equivariante $\quad 1$

1.1 Grupos de isometrias . . . . . . . . . . . . . . . . . . . 2

1.2 Espaço das órbitas . . . . . . . . . . . . . . . . . . . . . 4

1.3 Funcional de volume . . . . . . . . . . . . . . . . . . . . . 4

1.4 Teorema de redução . . . . . . . . . . . . . . . . . . . . 5

1.5 Ações polares . . . . . . . . . . . . . . . . . . . . . 10

2 Espaços simétricos não compactos $\quad 11$

2.1 Decomposição de Iwasawa . . . . . . . . . . . . . . . . . . . . . . . . . . . . 11

2.2 Posto maior do que um . . . . . . . . . . . . . . . . . . 13

2.2.1 Hipersuperfícies mínimas de $G / K$ dadas por hiperplanos de $\mathfrak{A}$. . . 13

2.2 .2 Hipersuperfícies mínimas e equivariantes de $G / K \ldots \ldots$. . . . . . 14

2.3 Posto um: o espaço hiperbólico quaterniônico . . . . . . . . . . . . . . . . 21

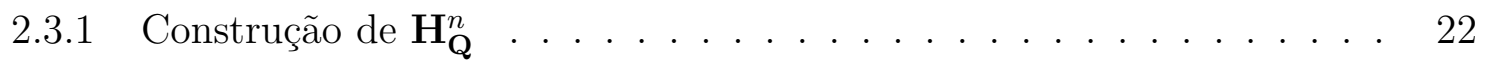

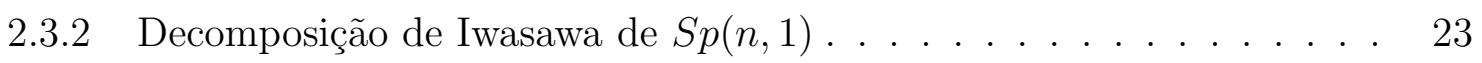

2.3.3 Modelos de $\mathbf{H}_{\mathbf{Q}}^{n} \ldots \ldots \ldots \ldots \ldots . \ldots \ldots . \ldots \ldots$

2.3.4 Bissetores e fans . . . . . . . . . . . . . . . . . . . 31

2.3.5 Hipersuperfícies mínimas . . . . . . . . . . . . . . . . 37

3 Plano projetivo octoniônico $\quad 53$

3.1 A álgebra dos octônions $\mathbf{O} \ldots \ldots \ldots \ldots \ldots$

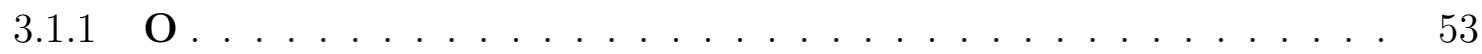

3.1 .2 Princípio de trialidade . . . . . . . . . . . . . 55

3.2 A álgebra de Jordan $\mathscr{J} \ldots \ldots \ldots \ldots \ldots$. . . . . . . . . . . . . . . . . . . . . . . . . . . . . . . .

$3.2 .1 \quad \mathrm{O}$ grupo $F_{4} \ldots \ldots \ldots \ldots \ldots \ldots$ 
3.2.2 O grupo $\operatorname{Spin}(9) \quad \ldots \ldots \ldots \ldots \ldots \ldots$

3.3 Modelo de Veronese . . . . . . . . . . . . . . . . . . . . . . . 60

3.4 Hipersuperfícies mínimas . . . . . . . . . . . . . . . . . . . . . . . 64

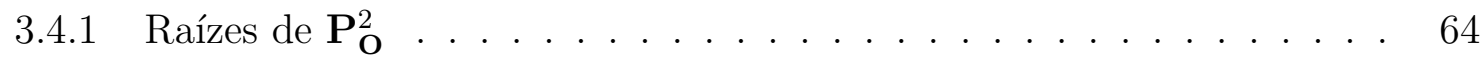

3.4 .2 Geometria de $\operatorname{Spin}(8), T^{1} \cdot \operatorname{Spin}(7)$ e $S U(2) \cdot S U(4) \ldots \ldots . . . . \quad 65$

3.4 .3 Análise da equação diferencial reduzida . . . . . . . . . . . . . . . . 73

Referências bibliográficas $\quad 85$

$\begin{array}{lr}\text { Índice remissivo } & 88\end{array}$ 
2.1 Soluções de $(2.5) \ldots \ldots$. . . . . . . . . . . . . . . . . . . 16

2.2 Soluções de (2.6) saindo ortogonalmente da fronteira $y=0 \ldots \ldots$. . . . . 21

2.3 Modelos do disco e do parabóloide para $\mathbf{H}_{\mathbf{Q}}^{n} \ldots \ldots \ldots \ldots . \ldots . \ldots 28$

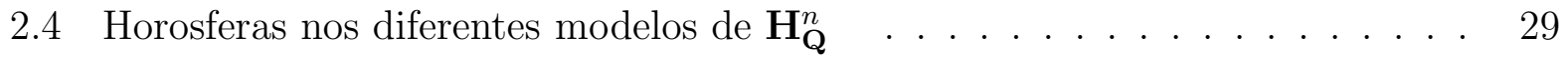

2.5 O feixe das geodésicas quaterniônicas que contêm $\left[x_{\infty}\right]$ na fronteira (no modelo do disco) . . . . . . . . . . . . . . . . . . . . . . 37

2.6 Soluções de (2.24) saindo ortogonalmente de $y=0 \ldots \ldots$. . . . . . . 43

2.7 Soluções de (2.26) saindo ortogonalmente de $y=0$, com $\mathbf{h} \equiv 0(a>0) \ldots . \quad 46$

2.8 Soluções de (2.29), para $\mathbf{h} \equiv 0$, saindo ortogonalmente de $\rho=0 \quad \ldots$. . . . 49

2.9 Soluções de $(2.30)$ para $\mathbf{h} \equiv 0 \ldots \ldots \ldots \ldots \ldots$. . . . . . . . . . . 51

3.1 Regiões determinadas por $f(r, \theta)=0 \ldots \ldots \ldots \ldots$

3.2 Padrão de pontos críticos da curva $\gamma_{a}$, para a perto de 0 (resp. perto de $\left.\arctan \sqrt{\frac{15}{7}}\right) \ldots \ldots \ldots \ldots \ldots \ldots$. . . . . . . . . . . . . . . . . 82

3.3 Soluções de (3.17) saindo (resp. chegando) ortogonalmente de $\theta=0$ (resp. em $\left.r=\frac{\pi}{2}\right) \ldots \ldots \ldots \ldots \ldots \ldots$. . . . . . . . . . . . . . . . . . 84 
Lista de tabelas

2.1 Expoentes dependendo do tipo de transformação . . . . . . . . . . . . . . . . 41

3.1 Expoentes dependendo do grupo . . . . . . . . . . . . . . . . . . . 73 
Dizemos que uma subvariedade imersa $\iota: N \rightarrow M$ de uma variedade riemanniana $M$ é mínima se seu campo de curvatura média, i.e. o traço da sua segunda forma fundamental, se anula identicamente. Seja $H$ é um grupo de Lie conexo que atua à esquerda sobre $M$. O espaço das $H$-órbitas $\tilde{M}=H \backslash M$ tem estrutura de variedade riemanniana estratificada. O conjunto $M_{P}$, dos pontos de $M$ cuja $H$-órbita é principal, é aberto e denso em $M$. Em particular, o subespaço das $H$-órbitas principais $\tilde{M}_{P}=H \backslash M_{P}$, está equipado, de maneira natural, com uma métrica riemanniana chamada métrica orbital, a qual é induzida pela distância entre as $H$-órbitas e é a única que torna a restrição $M_{P} \rightarrow \tilde{M}_{P}$, da projeção natural $M \rightarrow \tilde{M}$ (que leva cada ponto de $M$ na sua $H$-órbita), uma submersão riemanniana. Dizemos que a subvariedade imersa $\iota: N \rightarrow M$ é $H$-equivariante se $H$ age (à esquerda) sobre $N$ com $h(\iota(x))=\iota(h(x))$ para todo $h \in H$ e cada $x \in N$. Neste caso, obtemos a imersão induzida $\tilde{\iota}: \tilde{N} \rightarrow \tilde{M}$. Em [HL71] foi introduzido o denominado método da geometria diferencial equivariante. Este permite reduzir o problema de construir imersões mínimas no caso equivariante, a saber $\iota: N \rightarrow M$ é mínima se e somente se $\tilde{\iota}: \tilde{N} \rightarrow \tilde{M}$ é mínima em relação à métrica modificada. Esta métrica é conforme à métrica orbital por uma potência (que depende da cohomogeneidade de $\iota: N \rightarrow M$ com respeito a $(H, M)$ ) do funcional de volume, o qual é essencialmente o elemento de volume das $H$-órbitas principais ${ }^{1}$. O método pode ser levado eficazmente à pratica quando o problema reduzido consiste em construir geódesicas completas de uma superfície (que em geral tem bordo singular). Para isto, a cohomogeneidade de $(H, M)$ deve ser dois e, nesse caso, obtemos hipersuperfícies mínimas e completas de $M$. Tais geodésicas são soluções da equação diferencial reduzida (1.7) no Teorema 1.18.

Uma subvariedade conexa e completa $\Sigma$ de $M$ que intersecta todas as $H$-órbitas ortogonalmente é dita seção de $(H, M)$. Dizemos que o par $(H, M)$ é polar, ou que $H$ age polarmente sobre $M$, se este admite seções. O par $(H, M)$ diz-se hiperpolar se admite seções flat (com respeito à métrica induzida). Claramente todo par hiperpolar é polar. Para pares polares $(H, M)$, o espaço das $H$-órbitas $H \backslash M$ pode ser mergulhado isometricamente, de maneira natural, numa seção $\Sigma$, i.e. a métrica orbital coincide com a métrica induzida em

\footnotetext{
${ }^{1}$ Note que, em geral, as $H$-órbitas podem ser não compactas.
} 
$\Sigma$. Isto segue do fato de $\Sigma_{P}=\Sigma \cap M_{P}$ ser, necessariamente, uma variedade integral da distribuição horizontal da submersão riemanniana $M_{P} \rightarrow \tilde{M}_{P}$.

Como é bem conhecido, espaços simétricos são importantes exemplos de variedades riemannianas. De fato, o método da geometria diferencial equivariante tem sido uma ferramenta usada de maneira sistemática para construir hipersuperfícies mínimas neste tipo de variedade ambiente. Existem numerosos trabalhos que abordam este problema no caso de posto um e dois. Por exemplo, para o tipo compacto: [Hsi83a], [Hsi83b], [HS86], [Tom87] (problema de Bernstein esférico); [HHT88], [Tom90],[Tom87], [Tom96] (posto um); [HH80] e [HH82] (posto dois). Nestes são estudados pares polares $(H, M)$, onde $M=G / K$ é um espaço simétrico de tipo compacto e $H$ é um subgrupo fechado de $G$ que atua sobre $M$ com cohomogeneidade dois. Para espaços simétricos $M=G / K$ de tipo não-compacto [GG00], o subgrupo $H$ é adaptado à decomposição de Iwasawa $G=N A K$ de $G$. O par $(H, M)$ também é polar.

No presente trabalho, construiremos novos exemplos de hipersuperfícies (folheações em alguns casos) mímimas, completas e equivariantes de espaços simétricos. Dividiremos nosso estudo em duas partes, a saber, espaços simétricos de tipo compacto e não compacto.

\section{Tipo não compacto}

Em [GG00] são construídas hipersuperfícies (folheações) mínimas, completas e $H$-equivariantes do espaço hiperbólico complexo $\mathbf{H}_{\mathbf{C}}^{n}=S U(n, 1) / U(n)$, onde $H$ é um subgrupo adaptado à decomposição de Iwasawa de $S U(n, 1)$. É possível fazer um estudo análogo e mais geral seguindo as idéias de [GG00]. Seja $M=G / K$ um espaço simétrico de tipo não compacto. Consideremos a decomposição de Iwasawa $G=N A K$ da componente conexa da identidade $G$ do grupo de isometrias de $G / K$. Sejam $p$ e $q$ o posto (i.e. $p=\operatorname{dim} A$ ) e a dimensão de $G / K$, respectivamente.

Suponhamos primeiramente que $p>1$. Dado um subgrupo $A_{1} \subset A$ de codimensão dois, então o subgrupo $H=N A_{1}$ de $G$ atua hiperpolarmente sobre $G / K$ com cohomogeneidade dois. Com efeito, $H \backslash G / K=A_{2}$, onde $A=A_{1} A_{2}$ (note que se $p=2$, então $H=N$ ). Na outra mão, o grupo $N$ age hiperpolarmente sobre $G / K$ com cohomogeneidade $p$, de fato $N \backslash G / K=A$. Mostramos que:

Teorema 0.1. (i) Caso $H=N A_{1}, p \geq 2$

(a) Existe uma folheação $\mathscr{F}$ de $G / K$ por hipersuperfícies mínimas, completas, homogêneas, equidistantes e difeomorfas a $\mathbf{R}^{q-1}$, invariante por um grupo a um parâmetro de transvecções.

(b) Seja $\delta$ a metade da soma (incluindo as multiplicidades) das raízes (positivas) de $G / K$. Dado $d \geq \frac{\pi}{2|\delta|}$, seja $\mathscr{R}$ a região de $G / K$ delimitada por duas folhas $F_{1}, F_{2}$ de $\mathscr{F}$ separadas por uma distância d. Então existe uma folheação de $\mathscr{R}$ por hipersuperfícies minímas, completas, $H$-equivariantes, difeomorfas a $\mathbf{R}^{q-1}$ e assintóticas 
a $F_{1}$ e $F_{2}$, que é invariante por um grupo a um parâmetro de transveções (vide Figura 2.1).

(ii) Caso $H=N, p \geq 3$. Existe uma folheação de $G / K$ por hipersuperfícies mínimas, completas $N$-equivariantes e difeomorfas a $\mathbf{R}^{q-1}$, invariante por um grupo a um parâmetro de transvecções.

Agora consideremos $p=1$. Em [dCD83] e [GG00] foram introduzidos vários exemplos de hipersuperfícies mínimas (também de curvatura média constante), completas e equivariantes dos espaços hiperbólicos real e complexo $\mathbf{H}_{\mathbf{R}}^{n}$ e $\mathbf{H}_{\mathbf{C}}^{n}$, respectivamente. Nós generalizamos os resultados de [GG00] para o espaço hiperbólico quaterniônico $\mathbf{H}_{\mathbf{Q}}^{n}=S p(n, 1) / S p(n) \times S p(1)$. Com efeito, consideremos a decomposição de Iwasawa $S p(n, 1)=\mathscr{H}^{4 n-1} \cdot \mathbf{R}_{+} \cdot(S p(n) \cdot S p(1))$ de $S p(n, 1)$, onde $\mathscr{H}^{4 n-1}=\mathbf{Q}^{n-1} \times \Im(\mathbf{Q})$ é o grupo de Heisenberg generalizado. Consideramos três famílias de subgrupos de $S p(n, 1)$, adaptados à decomposição de Iwasawa, a saber elíptico, loxodrômico (loxodrômico especial) e parabólico (parabólico especial). Nos dois primeiros casos mostramos que:

Teorema 0.2. (i) Para cada $m=1, \ldots, n-1$, seja $H=S p(m) \times S p(n-m) \times\{1\}$ diagonalmente mergulhado no grupo de isometrias $S p(n, 1)$ de $\mathbf{H}_{\mathbf{Q}}^{n}$. Então existem infinitas hipersuperfícies minimas, completas, mergulhadas e não congruentes entre si em $\mathbf{H}_{\mathbf{Q}}^{n}$ que são H-equivariantes com cohomogeneidade um.

(ii) Para cada $m=2, \ldots, n-1$, seja $H=S p(n-m) \times\{1\} \times S p(m-1,1)$ diagonalmente mergulhado no grupo de isometrias $S p(n, 1)$ de $\mathbf{H}_{\mathbf{Q}}^{n}$. Então existem infinitas hipersuperfícies minimas, completas, mergulhadas e não congruentes entre si em $\mathbf{H}_{\mathbf{Q}}^{n}$ que são $H$-equivariantes com cohomogeneidade um.

Como é bem conhecido, $\mathbf{H}_{\mathbf{Q}}^{n}$ pode ser identificado com o disco aberto $\mathbf{D}^{n}$ de dimensão real $4 n$ de modo que sua fronteira ideal $\partial \mathbf{H}_{\mathbf{Q}}^{n}$ fica identificada com a esfera $\partial \mathbf{D}^{n}=\mathbf{S}^{4 n-1}$ (vide $\S 2.3 .3)$. Nesse caso, a fronteira ideal $\partial X$ de uma subvariedade mergulhada $X$ é definida por $\partial X=: \bar{X} \cap \partial \mathbf{D}^{n}$, onde $\bar{X}$ denota o fecho de $X$ em $\mathbf{D}^{n} \cup \partial \mathbf{D}^{n}$. Não é difícil ver que as hipersuperfícies construídas no Teorema 0.2 item (i) são difeomorfas ao produto $\mathbf{R}^{4 m} \times \mathbf{S}^{4 n-4 m-1}$ com a fronteira ideal homogênea e difeomorfa ao produto de Q-esferas $\mathbf{S}^{4 m-1} \times \mathbf{S}^{4 n-4 m-1}$.

Em geometria hiperbólica complexa são estudados, dentre vários outros objetos, dois tipos de hipersuperfícies mínimas; a saber bissetores e fans (vide [Gol06] e [GP92]), as quais estão fortemente relacionadas. Bissetores no espaço hiperbólico complexo foram introduzidos em [Mos80]. Em [AK07] são introduzidos bissetores para os espaços hiperbólicos quaterniônico e octoniônico. Um bissetor é definido como o lugar geométrico dos pontos equidistantes de dois pontos diferentes dados. Em $\S 2.3 .4$ estudamos bissetores em $\mathbf{H}_{\mathbf{Q}}^{n}$ e mostramos que estes são hipersuperfícies mínimas, regradas, de cohomogeneidade um², to-

\footnotetext{
${ }^{2} \mathrm{Em}$ [AK07] afirma-se, sem demonstração, que bissetores em $\mathbf{H}_{\mathbf{K}}^{n}, \operatorname{com} \mathbf{K}=\mathbf{R}, \mathbf{C}, \mathbf{Q}$ e $\mathbf{O}($ se $n=2)$, têm cohomogeneidade $\operatorname{dim}_{\mathbf{R}} \mathbf{K}-1$. Nos parece que houve ali um engano. Vide Exemplo 2.18, §2.3.4.
} 
dos congruentes entre si, difeomorfos a $\mathbf{R}^{4 n-1}$ e com fronteira ideal difeomorfa a $\mathbf{S}^{4 n-2}$. Bissetores têm uma estrutura geométrica bastante interessante, em particular apresentam vários tipos de decomposição. Nós destacamos a decomposição slice. Dados dois pontos distintos de $\mathbf{H}_{\mathbf{Q}}^{n}$, existe uma única geodésica quaterniônica, i.e. uma subvariedade totalmente geodésica isométrica a $\mathbf{H}_{\mathbf{Q}}^{1}$, contendo esses pontos. Consideremos o bissetor determinado por um par de pontos diferentes de $\mathbf{H}_{\mathbf{Q}}^{n}$. A geodésica quaterniônica contendo esses pontos diz-se Q-espinha do bissetor. A intersecção do bissetor com sua Q-espinha é chamada espinha do bissetor. A projeção ortogonal ${ }^{3}$ de $\mathbf{H}_{\mathbf{Q}}^{n}$ sobre a Q-espinha define uma folheação do bissetor com folhas isométricas a $\mathbf{H}_{\mathbf{Q}}^{n-1}$ chamadas slices do bissetor. A saber, cada slice é a preimagem, pela projeção ortogonal sobre a Q-espinha, de um ponto da espinha (vide Exemplo 2.18). Em particular todo bissetor fica completamente determinado pela sua espinha (vide Lema 2.17). A fronteira ideal da espinha chama-se esfera vortical.

Nós mostramos que bissetores são não rígidos como hipersuperfícies mínimas:

Teorema 0.3. Bissetores em $\mathbf{H}_{\mathbf{Q}}^{n}$ admitem deformações não triviais preservando a minimalidade. A saber, cada bissetor pertence a uma família analítica a um parâmetro de hipersuperfícies mínimas e completas tal que nenhum outro membro da família é um bissetor.

Nós introduzimos o conceito de fan em $\mathbf{H}_{\mathbf{Q}}^{n}$ seguindo as idéias de [GP92] para o caso complexo. Além do ponto base, em $\mathbf{H}_{\mathbf{Q}}^{n}$ (em geral nos espaços hiperbólicos) destaca-se um outro ponto que pertence à fronteira ideal. Tal ponto é chamado ponto no infinito e é denotado por $\infty$. O ponto no infinito é bastante útil para estudar a geometria de $\mathbf{H}_{\mathbf{Q}}^{n}$, em particular permite introduzir as denominadas coordenadas horosféricas. Um fan com vértice em $\infty$, pode ser visto como o limite de uma família a um parámetro de bissetores que contêm $\infty$ nas suas esferas vorticais. Tal família é obtida aplicando-se um grupo a um parâmetro de isometrias parabólicas que fixam $\infty$ a um bissetor contendo $\infty$ na sua esfera vortical (vide Exemplo 2.23). Em $§ 2.3 .4$ mostramos que fans são hipersuperfícies mínimas, homogêneas, regradas, todas congruentes entre si, difeomorfas a $\mathbf{R}^{4 n-1}$ e com fronteira ideal homeomorfa a $\mathbf{S}^{4 n-2}$.

Nós também construímos algumas folheações mínimas interessantes de $\mathbf{H}_{\mathbf{Q}}^{n}$ :

Teorema 0.4. (i) Para cada $m=1, \ldots, n-1$ existe uma folheação de $\mathbf{H}_{\mathbf{Q}}^{n}$ por hipersuperfícies minimas, completas e difeomorfas a $\mathbf{R}^{4 n-1}$. Esta folheação é invariante por um grupo a um parâmetro de transvecções e cada folha tem fronteira ideal homeomorfa a uma variedade pinçada de Hopf do tipo $(4 m-1,4 n-4 m-1)$.

(ii) Existe uma folheação de $\mathbf{H}_{\mathbf{Q}}^{n}$ por hipersuperfícies minimas, completas e difeomorfas a $\mathbf{R}^{4 n-1}$. Esta folheação é invariante por um grupo a um parâmetro de transvecções $e$ cada folha tem fronteira ideal homeomorfa a um buquê de duas esferas $\mathbf{S}^{4 n-2}$.

\footnotetext{
${ }^{3}$ Numa variedade riemanniana $M$ com curvatura seccional negativa, a função distância é estritamente convexa. Portanto, dada uma subvariedade totalmente geodésica $L$ de $M$, a aplicação que leva cada ponto $x \in M$ no ponto em $L$ mais próximo de $x$ está bem definida e é chamada a projeção ortogonal de $M$ sobre $L$.
} 
(iii) Existe uma folheação de $\mathbf{H}_{\mathbf{Q}}^{n}$ por hipersuperfícies minimas, completas, homogêneas, regradas e difeomorfas a $\mathbf{R}^{4 n-1}$, a saber, cada folha é um fan. Esta folheação é invariante por um grupo a um parâmetro de isometrias parabólicas e cada folha tem fronteira ideal homeomorfa à esfera $\mathbf{S}^{4 n-2}$.

Uma variedade pinçada de $H_{o p f}{ }^{4}$ do tipo $(k, l)$, onde $k$ e $l$ são inteiros positivos, é definida como sendo o espaço topológico obtido contraindo uma fibra do fibrado trivial $\mathbf{S}^{k} \times \mathbf{S}^{l} \rightarrow \mathbf{S}^{l}$. Por exemplo, para $k=l=1$ temos um toro pinçado.

As folheações de $\mathbf{H}_{\mathbf{Q}}^{n}$ no Teorema 0.4, induzem folheações singulares em $\partial \mathbf{H}_{\mathbf{Q}}^{n} \approx \mathbf{S}^{4 n-1}$ pinçadas no ponto no infinito, isto segue da construção na demonstração. Note que, uma congruência entre duas destas folheações induz um homeomorfismo entre as respectivas folheações na fronteira ideal. Portanto, as folheações no item (i) para $m=1, \ldots, n-1$ juntamente com as folheações nos ítens (ii) e (iii), são dois a dois não congruentes, pois os tipos de folha das folheações induzidas em $\partial \mathbf{H}_{\mathbf{Q}}^{n}$ são dois a dois não homeomorfos.

\section{Tipo compacto}

O espaço projetivo octoniônico $\mathbf{P}_{\mathbf{O}}^{2}=F_{4} / \operatorname{Spin}(9)$ é um dos espaços simétricos compactos excepcionais. Em contraste com os demais espaços projetivos, este tem sido pouco estudado. Em particular, conhecem-se poucos exemplos de hipersuperfícies mínimas. Em [Tom96] são construídas infinitas imersões mínimas $\mathbf{S}^{15} \hookrightarrow \mathbf{P}_{\mathbf{O}}^{2}$, não congruentes entre si e Spin(8)equivariantes. Em [PT99] foram classificados os subgrupos $H$ de $F_{4}$ que atuam polarmente sobre $\mathbf{P}_{\mathbf{O}}^{2}$ com cohomogeneidade dois, a saber $H=\operatorname{Spin}(8), T^{1} \cdot \operatorname{Spin}(7), S U(2) \cdot S U(4)$ e $S U(3) \cdot S U(3)$. Nosso propósito nesse sentido é continuar o trabalho começado em [Tom96], i.e. construir novos exemplos de hipersuperfícies mínimas e completas de $\mathbf{P}_{\mathbf{O}}^{2}$, as quais são equivariantes pelos grupos $T^{1} \cdot \operatorname{Spin}(7)$ e $S U(2) \cdot S U(4)$. O caso do grupo $S U(3) \cdot S U(3)$ não faz parte deste trabalho, pois as técnicas necessárias para construir soluções da respectiva equação diferencial reduzida são de natureza diferente às usadas aqui. Nosso resultado é o seguinte

Teorema 0.5. (i) Seja $H=T^{1} \cdot \operatorname{Spin}(7)$. Então, existem pelo menos duas hipersuperfícies mínimas, completas, mergulhadas e $H$-equivariantes de $\mathbf{P}_{\mathbf{O}}^{2}$ (não congruentes à esfera métrica). Estas são respectivamente difeomorfas aos produtos de esferas $\mathbf{S}^{14} \times \mathbf{S}^{1} e$ $\mathbf{S}^{9} \times \mathbf{S}^{6}$

(ii) Seja $H=S U(2) \cdot S U(4)$. Então, existem pelo menos duas hipersuperfícies minimas, completas, mergulhadas e $H$-equivariantes de $\mathbf{P}_{\mathbf{O}}^{2}$ (não congruentes à esfera métrica). Estas são respectivamente difeomorfas aos produtos de esferas $\mathbf{S}^{13} \times \mathbf{S}^{2}$ e $\mathbf{S}^{10} \times \mathbf{S}^{5}$.

Seja $M$ o espaço projetivo octoniônico $F_{4} / \operatorname{Spin}(9)$. Temos que os grupos $H$ no Teorema 0.5 ítens (i) e (ii) são subgrupos de $\operatorname{Spin}(9)$, portanto, fixam o ponto base $p \in M$. O

\footnotetext{
${ }^{4}$ Em inglês: pinched Hopf manifold.
} 
espaço das $H$-órbitas $\Delta=H \backslash M$ é isométrico a um triângulo geodésico na esfera $\mathbf{S}^{2}$, cujo interior corresponde às órbitas principais. Sejam $p$ (ponto base), $q$ e $q^{\prime}$ os pontos em $M$ correspondentes aos vértices de $\Delta$. Estamos interessados em soluções da equação diferencial reduzida de $(H, M)$ que começam e terminam, sem autointersecções, no interior de arestas diferentes de $\Delta$. Temos exatamente três classes de tal tipo de soluções. As correspondentes hipersuperfíces geradas são difeomorfas a os fibrados de esferas normais sobre as órbitas singulares $H(p), H(q)$ e $H\left(q^{\prime}\right)$, respectivamente. Temos que $H(p)=\{p\}$ entanto que $H(q)$ e $H\left(q^{\prime}\right)$ são difeomorfas a esferas. Usando fatos bem conhecidos de fibrados de esferas sobre esferas [Ste99], observamos que esses fibrados são triviais.

\section{A equação diferencial reduzida}

Tanto nos trabalhos citados anteriormente quanto no presente, o par $(H, M)$ é polar. Portanto, o espaço das $H$-órbitas $\Delta=H \backslash M$ está mergulhado de maneira natural em $M$ de tal forma que a métrica orbital é a métrica induzida (i.e. isometricamente). Temos que $\Delta$ é um orbifold (compacto ou não dependendo se $M$ o é ou não, respectivamente) de dimensão dois. Seu bordo $\partial \Delta$ é, em geral, não vazio e corresponde às órbitas singulares. Em todos os casos têm-se coordenadas naturais e globais (vide Observação 1.19(ii)), de maneira tal que a análise da equação diferencial reduzida tem uma abordagem geométrica. Estamos interessados em fazer uma análise qualitativa e global das soluções da equação (1.7) nos casos específicos concernentes a este trabalho. As soluções sempre são completas, portanto geram hipersuperfícies completas.

\section{Soluções saindo de $\partial \Delta$}

De fato, a equação (1.7) sempre é singular em $\partial \Delta$, pois o funcional de volume anula-se nos pontos de $\partial \Delta$ (vide Proposição 1.11). No entanto, sempre existem soluções emanando perpendicularmente de pontos de $\partial \Delta$ correspondentes a estratos de codimensão um. Tais soluções geram hipersuperfícies não singulares. A saber, tem-se o seguinte resultado:

Lema 0.6 (Vide [HH82] p.587 ou [Tom88] p.4). Seja $(H, M)$ qualquer um dos pares polares considerados nos teoremas 0.1(ii), 0.2, 0.3(i), 0.4 e 0.5. Consideremos o orbifold bidimensional $\Delta=H \backslash M$ com fronteira não vazia $\partial \Delta$. Suponhamos que $z_{0} \in \partial \Delta$ pertence a um estrato de codimensão um. Então, existe uma única solução $\gamma_{z_{0}}$ de (1.7) que é, necessariamente, perpendicular a $\partial \Delta$ em $z_{0}=\gamma_{z_{0}}(0)$. A hipersuperfície gerada é suave. Ainda mais, existe uma vizinhança de $\left(z_{0}, 0\right)$ em $\partial \Delta \times \mathbf{R}$ tal que $\gamma_{z}(s)=\gamma(z, s)$ é analítica.

Em geral, as soluções dadas pelo Lema 0.6 são as mais interessantes. Nós estudamos famílias a um parâmetro deste tipo de soluções. Estas podem eventualmente intersectar de novo $\partial \Delta$. No nosso caso, quando $M$ é de tipo não compacto isto nunca acontece, o qual simplifica consideravelmente a análise da equação (1.7). Na outra mão, quando $M$ é de tipo 
compacto, tanto no nosso quanto nos trabalhos citados, constroem-se soluções completas intersetando $\partial \Delta$ (perpendicularmente) em dois pontos distintos correspondentes a estratos de codimensão um (possivelmente diferentes). A construção de tais soluções torna-se um tanto mais difícil do que no caso de tipo não compacto. Para construí-las usam-se algumas estimativas que involucram as características geométricas específicas de (1.7), em cada caso particular.

\section{Soluções particulares e reflexões}

Na literatura encontramos vários métodos usados para construir soluções de (1.7) dependendo das características geométricas tanto do espaço das órbitas $\Delta$ quanto da equação diferencial em questão. De fato, quando $M$ é de tipo não compacto, a ferramenta mais usada para construir soluções é o método das primeiras e semi-primeiras integrais (vide [GG00], $\S 2.2 .2$ b) e $\S 2.3 .5)$. Por outro lado, algumas vezes temos simetrias de (1.7), a saber, relflexões em $\Delta$ com respeito a soluções explícitas. Tais reflexões reduzem consideravelmente o trabalho da construção de soluções. Nestes casos, constrói-se primeiramente um pedaço e a seguir usam-se as reflexões para obter uma solução completa (vide [HH82], [Tom90], [Tom96], e §2.3.5 c')). Também podem-se usar as reflexões para construir novas soluções a partir de soluções existentes ( vide $\S 2.3 .5$ b’)). Na outra mão, podemos ter translações que deixam (1.7) invariante, as quais permitem construir folheações (vide [GG00], §2.2.2 a) e b), $\S 2.3 .5$ c) e c')). Finalmente, pode acontecer o caso de existirem soluções explícitas mas não haver simetrias em relação a estas (vide §3.4.3).

\section{Equação de Jacobi ao longo de soluções explícitas}

Soluções de (1.7) são geodésicas em $\Delta$ com respeito à métrica modificada (vide Teorema 1.18). Como foi dito acima, as soluções explícitas são de grande ajuda para construir novas soluções. Em particular, a equação de Jacobi (em relação à métrica modificada) ao longo de tais soluções corresponde a uma linearização de (1.7). Portanto, aproxima soluções que estão perto de uma solução explícita. De fato, dada uma solução explícita $\gamma_{0}$, é de grande interesse saber o número de interseç̧ões de $\gamma_{0}$ com uma solução próxima (vide [HS86], [Tom87], [Tom96] e §3.4.3). Para tal, estima-se o número de zeros das soluções da equação de Jacobi (note que este é um fenômeno exclusivo de dimensão dois), isto involucra estimativas dadas pela Teoria de Sturm-Liouville.

\section{Comportamento das soluções perto dos vértices de $\Delta$}

Também é importante considerar o comportamento da equação (1.7) perto dos vértices de $\Delta$, i.e. os pontos de $\partial \Delta$ correspondentes a estratos de codimensão dois. Dado um vértice de $\Delta$, este corresponde a um ponto singular de tipo isolado ${ }^{5} p$ de $(H, M)$. Nesse caso,

\footnotetext{
${ }^{5} \mathrm{Ou}$ seja, existe uma vizinhança de $p$ onde pontos diferentes de $p$ têm tipos de órbita diferente de $H(p)$.
} 
é possível estudar o comportamento de soluções de (1.7) perto de $p$. Para isto, fixamos uma vizinhança normal $\mathscr{U}$ de $p$ e consideramos uma família (analítica) a um parâmetro de homotetias definidas em $\mathscr{U}$, i.e. difeomorfismos que transformam esferas métricas (com centro em $p$ ) em esferas métricas de raio menor. Esta induze uma família de métricas sobre $\mathscr{U}$ (a saber, tais métricas são normalizações dos pull-backs da métrica ambiente com respeito às homotetias) de tal forma que a métrica limite é a métrica flat em $\mathscr{U}$ dada pela métrica ambiente no ponto $p$. Para cada valor do parâmetro obtemos um problema equivariante equivalente ao inicial. A equação diferencial reduzida limite é mais simples, a saber podese reduzir a uma equação planar autônoma (vide [Hsi83a], [Hsi83b] e §3.4.3). Os pontos singulares do sistema planar correspondem às condições inicias do problema original para soluções saindo de (estratos de codiensão um em) $\partial \Delta$, assim como a soluções explícitas que convergem a $p$. O comportamento global das soluções da equação diferencial reduzida limite aproxima, via as homotetias, o comportamento local, perto de $p$, das soluções do problema original.

Finalmente, queremos fazer notar que o principal obstáculo na nossa análise da equação diferencial reduzida para o caso de $\mathbf{P}_{\mathbf{O}}^{2}$ (vide equação 3.21) é o fato de que, embora existam soluções explícitas, se dá o caso da não existência de simetrias (compare §3.4.3 com [Tom96]). Na outra mão, como foi dito acima, os métodos usados para o caso $H=S U(3) \cdot S U(3)$ são diferentes dos considerados aqui. Isto vem do fato de não existirem soluções explícitas. Para saber mais a respeito desses métodos pode-se consultar [Gor94] e [Gor96].

\section{Estrutura do trabalho}

Dividimos esta monografia em três capítulos. O Capítulo 1 está constituído por cinco seções. Em $\S 1.1$ e $\S 1.2$ apresentamos (sem demonstração) os fatos básicos da teoria de ações isométricas. Em $\S 1.3$ definimos o funcional de volume. Em $§ 1.4$ refazemos a demonstração do Teorema de redução (Teorema 1.18), que apresenta a equação diferencial reduzida (1.7). Este será usado de maneira sistemática para a obtenção dos nossos resultados. Finalmente, em $§ 1.5$ recordamos algumas idéias básicas da teoria de ações polares.

O Capítulo 2 está dividido em três seções. Em $§ 2.1$ apresentamos (sem demonstração) a decomposição de Iwasawa de um grupo de Lie não compacto e semi-simples. Em $\S 2.2$ demonstramos o Teorema 0.1. A seção $\S 2.3$ divide-se em cinco partes. Em $\S 2.3 .1$ construímos o espaço hiperbólico quaterniônico. Em $§ 2.3 .2$ deduzimos a decomposição de Iwasawa para $S p(n, 1)$. Em $§ 2.3 .3$ são construídos os diferentes modelos de $\mathbf{H}_{\mathbf{Q}}^{n}$ e são apresentados os aspectos elementares da geometria de $\mathbf{H}_{\mathbf{Q}}^{n}$. Em $\S 2.3 .4$ expomos os fatos básicos acerca de bissetores, introduzimos fans e mostramos a relação existente entre estes dois objetos. Finalmente, em $\S 2.3 .5$ demonstramos os teoremas 0.2 , 0.3 e 0.4 .

Por último, o Capítulo 3 consta de quatro seções. Em $§ 3.1$ expomos as propriedades álgebricas básicas dos octônions e enunciamos o Princípio de trialidade. Em §3.2 estudamos 
a álgebra de Jordan que dá origem ao grupo excepcional $F_{4}$ e discutimos a relação de $F_{4}$ com $\operatorname{Spin}(9)$ e $\operatorname{Spin}(8)$. Em $§ 3.3$ construímos o modelo de Veronese para $\mathbf{P}_{\mathbf{O}}^{2}$, o qual usaremos para realizar alguns dos cômputos necessários para demonstrar o Teorema 0.5. Finalmente, em $§ 3.4$ demonstramos o Teorema 0.5.

Ao longo deste trabalho apresentamos vários resultados bem conhecidos e, portanto, sem demonstração. No entanto, citamos, em cada caso, pelo menos uma referência onde o leitor encontrará tanto a prova quanto uma discussão mais ampla dos temas relativos ao resultado em questão. Supomos também que o leitor está familiarizado com a teoria de espaços simétricos. Para uma breve introdução pode-se consultar [Arv03]. Para um estudo mais profundo vide [Hel78] ou [Kna96] 


\section{CAPÍTULO 1}

\section{Geometria diferencial equivariante}

Do ponto de vista da geometria diferencial, o problema (local) de construir subvariedades mínimas de uma variedade riemanniana dada é equivalente ao de construir soluções da seguinte equação diferencial em derivadas parciais

$$
\operatorname{tr}(I I)=0
$$

onde II denota a segunda forma fundamental da subvariedade. O método da geometria diferencial equivariante [HL71] nos permite reduzir este problema a um do mesmo tipo, e por sua vez equivalente; mas em dimensão, tanto da variedade ambiente quanto da subvariedade a ser construída, menor. Para isso, acrescentamos à condição de ser mínima a de ser equivariante em relação a um subgrupo (fechado) do grupo de isometrias da variedade ambiente, i.e. dito subgrupo age sobre a subvariedade pela restrição da ação na variedade ambiente. O método pode ser levado eficazmente à prática se o problema original é reduzido a estudar uma equação diferencial ordinária, a saber definida numa superfície, para assim construir hipersuperfícies mínimas. Ainda mais, podemos fazer um estudo global (construir soluções completas) e assim obter hipersuperfícies completas.

O próposito principal deste capítulo é refazer a demonstração do Teorema de redução (Teorema 1.18). Para tal, devemos lembrar alguns conceitos básicos da teoria de ações isométricas. Na outra mão, também recordaremos alguns resultados da teoria de ações polares. Nos dois capítulos restantes usamos o Teorema de redução sistematicamente para obter nossos resultados. 


\subsection{Grupos de isometrias}

Sejam $\left(M, d s^{2}\right)$ uma variedade riemanniana completa e $H$ um subgrupo fechado e conexo do grupo de isometrias Iso $(M)$ de $M$. Dado $x \in M$ a $H$-órbita $H(x)$ e o subgrupo de isotropia $H_{x}$ de $x$, são definidos respectivamente por

$$
H(x)=\{h(x): h \in H\} \quad \text { e } \quad H_{x}=\{h \in H: h(x)=x\} .
$$

Temos que $H_{x}$ é um subgrupo fechado de $H$, portanto o espaço topológico quociente $H / H_{x}$ é de Hausdorff e tem uma única estrutura de variedade diferenciável tal que a aplicação $\phi_{x}$ dada por

$$
\begin{aligned}
\phi_{x}: H / H_{x} & \rightarrow M \\
h \cdot H_{x} & \mapsto h(x)
\end{aligned}
$$

é uma imersão. Esta por sua vez induz uma bijeção de $H / H_{x}$ sobre a sua imagem $H(x)$. Segue que $H(x)$ é uma subvariedade imersa de $M$.

Duas $H$-órbitas são ditas do mesmo tipo se os subgrupos de isotropia correspondentes são conjugados em $H$. As classes de conjugação $\left\{\left(H_{x}\right): x \in M\right\}$ são chamadas tipos de isotropia do par $(H, M)$. Podemos ordenar parcialmente os tipos de isotropia assim:

$$
\left(H_{y}\right) \preceq\left(H_{x}\right) \text { se e somente se } H_{x} \text { é conjugado em } H \text { a um subgrupo de } H_{y} \text {. }
$$

Definição 1.1. Dizemos que a órbita $H(x)$ é principal se $\left(H_{x}\right)$ é maximal em relação à ordem parcial $\preceq$. Nesse caso, o ponto $x$ é chamado regular. Se a $H$-órbita de $x$ não é principal mas tem dimensão maximal, $H(x)$ é chamada órbita excepcional. As demais órbitas são chamadas singulares. Os pontos não regulares são ditos pontos singulares. Denotamos por $M_{P}$ o conjunto dos pontos regulares.

O fato de $H_{x}$ fixar $x$ implica que $H_{x}$ atua naturalmente sobre $T_{x} H(x)$ por $(h, X) \mapsto h_{*} X$, com $h \in H_{x}$ e $X \in T_{x} H(x)$ (onde $h_{*}$ denota a diferencial de $h$ ). Ainda, como

$$
d s^{2}\left(h_{*} X, h_{*} Y\right)=d s^{2}(X, Y),
$$

para todo par de vetores $X, Y \in T_{x} M$ (pois os elementos de $H$ são isometrias), temos que $H_{x}$ age também sobre o espaço normal $\nu_{x} H(x)$ a $H(x)$ em $x$.

Definição 1.2. As ações de $H_{x}$ sobre $T_{x} H(x)$ e $\nu_{x} H(x)$ são chamadas ação isotrópica e slice, respectivamente.

Proposição 1.3 (Vide [PT88],[Gor10]). Uma H-órbita é principal se e somente se a ação slice correspondente é trivial. 
Corolário 1.4. Toda H-órbita principal é orientável. Ainda mais, seu fibrado normal é trivial.

Demonstração. Seja $H(x)$ uma órbita principal. Dado $\xi \in \nu_{x} H(x)$ temos que $\hat{\xi}_{h(x)}=$ : $h_{*} \xi$ define um campo normal $H$-equivariante de $H(x)$, globalmente definido. Com efeito, basta mostrar que está bem definido. Isto segue do fato da ação slice $\left(H_{x}, \nu_{x} H(x)\right)$ ser trivial. Finalmente, uma base de $\nu_{x} H(x)$ define um referencial global de $\nu H(x)$ como foi feito acima.

Observação 1.5. Sejam $M$ e $N$ duas variedades riemannianas e $H$ um grupo de Lie. Suponhamos que $H$ atue à esquerda sobre $M$ e $N$. Dizemos que uma aplicação diferenciável $\psi: N \rightarrow M$ é $H$-equivariante se $h(\psi(x))=\psi(h(x))$, para todo $h \in H$ e cada $x \in N$.

(i) Sejam $x, y \in M$ tais que $\left(H_{y}\right) \preceq\left(H_{x}\right)$. Então existe $g \in H$ tal que $g H_{x} g^{-1}$ é um subgrupo de $H_{y}$. Assim a aplicação

$$
\begin{aligned}
\psi: H(x) & \rightarrow H(y) \\
h(x) & \mapsto g h g^{-1}(y),
\end{aligned}
$$

define o fibrado riemanniano $H$-equivariante

$$
H_{y} / H_{x} \hookrightarrow H(x) \longrightarrow H(y)
$$

(ii) Seja $x \in M$. Temos que $H_{x}$ age de naturalmente sobre o produto $H \times \nu_{x} H(x)$ assim

$$
k(h, \xi)=\left(h k^{-1}, k_{*} \xi\right)
$$

(isto é, translações à esquerda e ação slice no primeiro e segundo fator, respectivamente) para todo $k \in H_{x}$ e cada $\xi \in \nu_{x} H(x)$. O espaço quociente e denotado por $H \times_{H_{x}} \nu_{x} H(x)$. Ainda mais temos o fibrado vectorial $H$-homogêneo

$$
\nu_{x} H(x) \hookrightarrow H \times_{H_{x}} \nu_{x} H(x) \longrightarrow H(x)
$$

(iii) De fato, os dois fibrados anteriores estão relacionados com o seguinte fibrado mais simples. Dado $x \in M$ a aplicação $H \rightarrow H(x)$, dada por $h \mapsto h(x)$, para todo $h \in H$, define o fibrado

$$
H_{x} \hookrightarrow H \longrightarrow H(x)
$$

A existência de seções locais para este fibrado nos permite construir trivializações locais para os fibrados dos ítens (i) e (ii). 
Proposição 1.6 (Vizinhança tubular (vide [PT88] ou [Dui00])). Seja $x \in$ M. Existe uma vizinhança tubular de $H(x)$ difeomorfa a $H \times_{H_{x}} \nu_{x} H(x)$. Ainda mais, este difeomorfismo é $H$-equivariante.

\subsection{Espaço das órbitas}

A ação de $H$ sobre $M$ induz uma partição de $M$ dada pelas $H$-órbitas. Seja $\tilde{M}=H \backslash M$, dizemos que $\tilde{M}$ é o espaço das órbitas. $\tilde{M}$ munido com a topologia quociente é um espaço de Hausdorff e tem uma estrutura natural de espaço métrico, onde a distância entre dos pontos em $\tilde{M}$ é dada pela distância entre as respectivas $H$-órbitas em $M$. Consideremos a projeção canônica $\pi: M \rightarrow \tilde{M}$ que leva cada ponto $x \in M$ na sua $H$-órbita $\pi(x)=H(x)$. Denotemos por $\tilde{M}_{P}$ o conjunto das órbitas principais $H \backslash M_{P}=\pi\left(M_{P}\right)$. O conjunto $\tilde{M}_{P}$ tem estrutura natural de variedade riemanniana, mais precisamente, existe uma única métrica riemanniana $d \tilde{s}^{2}$ definida em $\tilde{M}_{P}$ que torna a restrição $\left.\pi\right|_{P}: M_{P} \rightarrow \tilde{M}_{P}$ uma submersão riemanniana. Dizemos que $d \tilde{s}^{2}$ é a métrica orbital. A distância induzida pela métrica orbital dá a estrutura de espaço métrico descrita acima.

Proposição 1.7 (Teorema da órbita principal (Vide [PT88], [Dui00] ou [Gor10])). Seja M conexa. Então existe um único tipo de órbita principal. Ainda mais, $M_{P}$ é aberto e denso em $M$ e $\tilde{M}_{P}$ é conexo.

Segue da proposição acima que todas as órbitas principais são difeomorfas e portanto têm a mesma dimensão. A codimensão das órbitas principais coincide com a dimensão de $M_{P}$.

Definição 1.8. A codimensão comum das $H$-órbitas principais de $M$ é chamada cohomogeneidade do par $(H, M)$.

\subsection{Funcional de volume}

Denotemos por $\mathfrak{H}$ a álgebra de Lie de $H$. Dado $X \in \mathfrak{H}$ o campo de Killing $X^{*}$ induzido por $X$ sobre $M$ é dado por

$$
X^{*}(x)=\left.\frac{d}{d t}\right|_{t=0} \exp (t X)(x)
$$

para todo $x \in M$. Seja $x \in M$ e denotemos por $\mathfrak{H}_{x}$ a álgebra de Lie de $H_{x}$. Então $X^{*}(x)=0$ se e somente se $X \in \mathfrak{H}_{x}$. Suponhamos que sobre $H$ existe uma métrica $\operatorname{Ad}_{H}$ invariante e consideremos a decomposição ortogonal

$$
\mathfrak{H}=\mathfrak{H}_{x}+\mathfrak{H}_{x}^{\perp}
$$

Nesse caso a diferencial da aplicação (1.1) é um isomorfismo de $\mathfrak{H}_{x}^{\perp}$ sobre $T_{x} H(x)$, tal que $\phi_{x *} X=X^{*}(x)$, para todo $X \in \mathfrak{H}_{x}^{\perp}$. 
Suponhamos que $H(x)$ é uma órbita principal. Pelo Corolário $1.4 H(x)$ é orientável. Sejam $\Omega_{x}$ e $\omega_{x}$ as formas elemento de volume de $H / H_{x}$ e $H(x)$, respectivamente, então existe uma função diferenciável $\mathscr{V}_{x}$ tal que

$$
\phi_{x}^{*} \omega_{x}=\mathscr{V}_{x} \Omega_{x}
$$

Proposição 1.9. A função $\mathscr{V}_{x}$ é constante. Ainda mais $\mathscr{V}_{x}$ pode ser calculada como segue. Seja $\left\{X_{1}, \ldots, X_{n}\right\}$ uma base ortonormal de $\mathfrak{H}_{x}^{\perp}$ e considere a matriz $A(x)=\left(a_{i j}(x)\right)$, onde $a_{i j}(x)=d s^{2}\left(X_{i}^{*}(x), X_{j}^{*}(x)\right)$, para todo $i, j=1, \ldots, n$. Então

$$
\mathscr{V}_{x}=\sqrt{\operatorname{det} A(x)}
$$

Demonstração. Temos que $\Omega_{x}$ e $\omega_{x}$ são formas $H$-invariantes, portanto de (1.2) segue que $\mathscr{V}_{x}$ é constante. A equação (1.3) segue diretamente ao computar o lado direito de (1.2) nos vetores $X_{1}, \ldots, X_{n}$.

Definição 1.10. O funcional de volume do par $(H, M)$ é a função $\mathscr{V}: \tilde{M} \rightarrow \mathbf{R}$ dada por

$$
\mathscr{V}(\tilde{x})= \begin{cases}\mathscr{V}_{x}, & \text { se } \tilde{x}=H(x) \text { é principal } \\ \sharp\left(H_{x} / L\right) \mathscr{V}_{x}, & \text { se } \tilde{x}=H(x) \text { é excepcional } \\ 0, & \text { caso contrário }\end{cases}
$$

sendo $(L)$ o tipo de isotropia principal de $(H, M)$.

Proposição 1.11 (Vide [HL71]). A função $\mathscr{V}$ é contínua em $\tilde{M}$, diferenciável em $\tilde{M}_{P} e$ anula-se nas órbitas singulares não excepcionais.

Observação 1.12. Um resultado recente em [Ale11] mostra que, se $M$ é simplesmente conexa e $(H, M)$ é polar, então não existem órbitas excepcionais. Esse e o caso dos exemplos estudados neste trabalho. Dessa maneira, o funcional de volume é mais simples de calcular.

\subsection{Teorema de redução}

Definição 1.13. Dizemos que uma subvariedade (imersa) $\iota: N \rightarrow M$ é $H$-equivariante se $H$ atua sobre $N$ de maneira tal que

$$
h(\iota(y))=\iota(h(y))
$$


para todo $h \in H$ e cada $y \in N$. A cohomogeneidade de $N$ em relação a $(H, M)$ é a dimensão da subvariedade (imersa)

$$
\begin{aligned}
\tilde{\iota}: \tilde{N}_{P} & \rightarrow \tilde{M}_{P} \\
H(y) & \mapsto H(\iota(y))
\end{aligned}
$$

para todo $y \in N_{P}$.

Observação 1.14. Note que o campo de curvatura média de uma subvariedade $H$-equivariante $\iota: N \rightarrow M$, i.e. o traço $\operatorname{tr}(I I)$ da sua segunda forma fundamental $I I$, é $H$-equivariante, ou seja

$$
h_{*} \operatorname{tr}\left(I I_{x}\right)=\operatorname{tr}\left(I I_{h(x)}\right),
$$

para todo $x \in M$ e cada $h \in H$. Isto mostra que $\operatorname{tr}(I I)$ é normal às $H$-órbitas. Portanto a restrição de $\operatorname{tr}(I I)$ a $M_{P}$ é horizontal em relação à submersão riemanniana $\pi_{P}: M_{P} \rightarrow \tilde{M}_{P}$.

Sejam $I I$ e $\tilde{I I}$ as segundas formas fundamentais de $N$ e $\tilde{N}$, respectivamente. Seja $c$ a cohomogeneidade de $N$ e consideremos a métrica $d \bar{s}^{2}=\mathscr{V}^{\frac{2}{c}} d \tilde{s}^{2}$ definida sobre $\tilde{M}_{P}$. Dizemos que $d \bar{s}^{2}$ é a métrica modificada. Denotemos com $\overline{I I}$ a segunda forma fundamental de $\tilde{N}$ em relação à métrica modificada.

Lema 1.15. Seja $\tilde{\xi}$ um campo unitário normal a $\tilde{N}$ e consideremos o seu levantamento horizontal $\xi$. Então

$$
d s^{2}(\operatorname{tr}(I I), \xi)=d \tilde{s}^{2}(\operatorname{tr}(\tilde{I I}), \tilde{\xi})-\tilde{\xi} \ln (\mathscr{V})
$$

Demonstração. Seja $\left\{\tilde{E}_{1}, \ldots, \tilde{E}_{k}\right\}$ um referencial ortonormal local de $T \tilde{N}_{P}$. Consideremos seu levantamento horizontal $\left\{E_{1}, \ldots, E_{k}\right\}$. Seja $L$ um subgrupo de isotropia principal. Consideremos uma base ortonormal $\left\{X_{1}, \ldots, X_{n}\right\}$ de $\mathfrak{L}^{\perp}$. Sejam $V_{1}, \ldots, V_{n}$ campos ortonormais tais que $X_{i}^{*}=\sum_{r} b_{i r} V_{r}$, para todo $i=1, \ldots, n$. Sejam $A=\left(a_{i j}\right)$ a matriz dada por (1.3), $B=\left(b_{i j}\right), A^{-1}=\left(a^{i j}\right)$ e $B^{-1}=\left(b^{i j}\right)$. Temos que

$$
\operatorname{tr}(I I)=\sum_{i} I I\left(E_{i}, E_{i}\right)+\sum_{j} I I\left(V_{j}, V_{j}\right)
$$

Projetando em $\xi$ obtemos $d s^{2}(\operatorname{tr}(I I), \xi)=d \tilde{s}^{2}(\operatorname{tr}(\tilde{I I}), \tilde{\xi})+\sum_{j} d s^{2}\left(I I\left(V_{j}, V_{j}\right), \xi\right)$. Vejamos que $\sum_{j} d s^{2}\left(I I\left(V_{j}, V_{j}\right), \xi\right)=-\frac{1}{2} \tilde{\xi} \ln (\operatorname{det} A)$. Com efeito, $I I\left(V_{j}, V_{j}\right)=\sum_{r, t} b^{j r} b^{j t} I I\left(X_{r}^{*}, X_{t}^{*}\right)$ e 


$$
\begin{aligned}
& d s^{2}\left(I I\left(X_{r}^{*}, X_{t}^{*}\right), \xi\right)=-\frac{1}{2} \xi d s^{2}\left(X_{r}^{*}, X_{t}^{*}\right)=-\frac{1}{2} \xi a_{r t}, \log 0 \\
& \sum_{j} d s^{2}\left(I I\left(V_{j}, V_{j}\right), \xi\right)=-\frac{1}{2} \sum_{j, r, t} b^{j r} b^{j t} \xi a_{r t} \\
&=-\frac{1}{2} \sum_{r, t}\left(\sum_{j} b^{j r} b^{j t}\right) \xi a_{r t} \\
&=-\frac{1}{2} \sum_{r, t} a^{r t} \xi a_{r t} \\
&=-\frac{1}{2} \sum_{r, t} a^{t r} \xi a_{r t} \\
&=-\frac{1}{2} \tilde{\xi} \ln (\operatorname{det} A) .
\end{aligned}
$$

O seguinte é um resultado geral sobre métricas conformes.

Lema 1.16. Seja $M$ uma variedade diferenciável. Consideremos duas métricas riemannianas $d s^{2}$ e d $\bar{s}^{2}$ definidas em $M$, onde $d \bar{s}^{2}=f^{2} d s^{2}$, com $f: M \rightarrow \mathbf{R}$ sendo uma função diferenciável e positiva; i.e. $d \bar{s}^{2}$ é conforme a $d s^{2}$. Suponhamos que $N$ é uma subvariedade de $M$ e sejam II e $\overline{I I}$ as segundas formas fundamentais de $N$ em relação a ds ${ }^{2}$ e $\bar{s}^{2}$, respectivamente. Então

$$
f^{2} \operatorname{tr}(\overline{I I})=\operatorname{tr}(I I)-\operatorname{dim} N \operatorname{grad}(\ln f)^{\perp},
$$

onde $\operatorname{grad} e(\cdot)^{\perp}$ denotam o gradiente e a projeção ortogonal sobre o fibrado normal de $N$ com respeito a $\mathrm{ds}^{2}$, respectivamente.

Demonstração. Denotemos com $\nabla$ e $\bar{\nabla}$ as conexões de Levi-Civita de $M$ com respeito a $d s^{2}$ e $\bar{d}^{2}$, respectivamente. Assim da fórmula de Koszul segue que se $X, Y, Z$ são campos tangentes a $M$, então

$$
d \bar{s}^{2}\left(\bar{\nabla}_{X} Y, Z\right)=f^{2} d s^{2}\left(\nabla_{X} Y, Z\right)+f\left\{X(f) d s^{2}(Y, Z)+Y(f) d s^{2}(X, Z)-Z(f) d s^{2}(X, Y)\right\}
$$

Agora, consideremos os referenciais (locais) $d s^{2}$-ortonormais tangente e normal $\left\{E_{1}, \ldots, E_{p}\right\}$ e $\left\{\xi_{1}, \ldots \xi_{q}\right\}$ a $N$, respectivamente. Então fazendo $\bar{E}_{i}=\frac{1}{f} E_{i}$ e $\bar{\xi}_{j}=\frac{1}{f} \xi_{j}$, obtemos respectivos referenciais $d \bar{s}^{2}$-ortonormais. Note que

$$
f^{2} d s^{2}\left(\nabla_{\bar{E}_{i}}, \bar{E}_{i}, \xi_{j}\right)=d s^{2}\left(\nabla_{E_{i}} E_{i}, \xi_{j}\right)
$$


Assim, usando isto junto com (1.4) segue que

$$
\begin{aligned}
f^{2} \operatorname{tr}(\overline{I I}) & =f^{2} \sum_{j, i} d \bar{s}^{2}\left(\bar{\nabla}_{\bar{E}_{i}} \bar{E}_{i}, \bar{\xi}_{j}\right) \bar{\xi}_{j} \\
& =\sum_{j, i} d \bar{s}^{2}\left(\bar{\nabla}_{\bar{E}_{i}} \bar{E}_{i}, \xi_{j}\right) \xi_{j} \\
& =\sum_{j, i}\left\{f^{2} d s^{2}\left(\nabla_{\bar{E}_{i}} \bar{E}_{i}, \xi_{j}\right)-f \xi_{j}(f) d s^{2}\left(\bar{E}_{i}, \bar{E}_{i}\right)\right\} \xi_{j} \\
& =\sum_{j, i}\left\{d s^{2}\left(\nabla_{E_{i}} E_{i}, \xi_{j}\right)-\xi_{j}(\ln f)\right\} \xi_{j} \\
& =\operatorname{tr}(I I)-\operatorname{dim} N \operatorname{grad}(\ln f)^{\perp} .
\end{aligned}
$$

Agora voltemos à notação prévia ao Lema 1.16.

Proposição 1.17. Seja $N$ uma subvariedade de $M$ com cohomogeneidade c respeito a $(H, M)$. Consideremos a projeção canônica $\pi: M \rightarrow \tilde{M}$ e seja $\tilde{N}=\pi(N)$. Então

$$
\pi_{*} \operatorname{tr}(I I)=\operatorname{tr}(\tilde{I I})-(\operatorname{grad}(\ln \mathscr{V}))^{\perp}
$$

Em particular $N$ é mínima se e somente se $\tilde{N}$ é mínima em relação à métrica modificada.

Demonstração. Pela Observação $1.14 \operatorname{tr}(I I)$ é um campo horizontal. Assim a equação (1.5) segue do Lema 1.15. Na outra mão a métrica modificada é conforme à métrica orbital, a saber

$$
d \bar{s}^{2}=\left(\mathscr{V}^{\frac{1}{c}}\right)^{2} d \tilde{s}^{2}
$$

Portanto, do Lema 1.16

$$
\operatorname{tr}(\tilde{I I})-\operatorname{grad}(\ln \mathscr{V})^{\perp}=\mathscr{V}^{\frac{2}{c}} \operatorname{tr}(\overline{I I})
$$

Combinando (1.5) e (1.6) segue a segunda afirmação.

Como corolário da Proposição 1.17 temos o resultado principal deste capítulo:

Teorema 1.18 (Teorema de redução). Seja H um grupo de Lie conexo agindo isometricamente (à esquerda), com cohomogeneidade dois, sobre uma variedade riemanniana $\left(M, d s^{2}\right)$. Seja $\Gamma$ uma hipersuperfície $H$-equivariante de $M$ com curvatura media $\mathbf{h}$. Suponhamos que $\Gamma$ tenha cohomogeneidade $c=1$ com respeito a $(H, M)$. Seja $\gamma$ a curva em $\tilde{M}=H \backslash M$ com $H \backslash \Gamma=\gamma$. Denotemos com $\kappa_{g}$ a curvatura geodésica de $\gamma$ em relação à métrica orbital d $\tilde{s}^{2}$ e com $\xi$ o campo normal unitário ao longo de $\gamma$. Então

$$
\mathbf{h}=\kappa_{g}-\frac{d}{d \xi} \ln (\mathscr{V})
$$

onde $\mathscr{V}$ é o funcional de volume de $(H, M)$. Em particular $\Gamma$ é minima se e somente se $\gamma$ é uma geodésica em relação à métrica modificada $d \bar{s}^{2}=\mathscr{V}^{2} d \tilde{s}^{2}$. 
Demonstração. Com efeito, nesse caso a equações (1.5) e (1.7) são equivalentes. Na outra mão, seja $\bar{\kappa}_{g}$ a curvatura geodésica de $\gamma$ em relação à métrica modificada $d \bar{s}^{2}$. Então a equação (1.6) transforma-se em

$$
\kappa_{g}-\frac{d}{d \xi} \ln (\mathscr{V})=\mathscr{V}^{2} \bar{\kappa}_{g}
$$

Combinando (1.7) e (1.8) e fazendo $\mathbf{h} \equiv 0$ segue a segunda afirmação.

Observação 1.19. (i) As ações consideradas neste trabalho são polares, assim $\tilde{M}$ está isometricamente mergulhado em $M$ (vide $\$ 1.5$ ). Portanto a métrica orbital $d \tilde{s}^{2}$ é a restição, a uma seção de $(H, M)$, da métrica ambiente $d s^{2}$. Logo também denotaremos a métrica orbital por $d s^{2}$.

(ii) Sejam $(x, y)$ são coordenadas globais para $\tilde{M}$ tais que $d s^{2}=E d x^{2}+G d y^{2}$. Seja $\gamma(s)=(x(s), y(s))$ uma curva em $\tilde{M}$ parametrizada pelo comprimento de arco. Então $\frac{d \gamma}{d s}=\frac{d x}{d s} \frac{\partial}{\partial x}+\frac{d y}{d s} \frac{\partial}{\partial y}$. Assim, se $\sigma$ é o ângulo entre $\frac{\partial}{\partial x}$ e $\frac{d \gamma}{d s}$, temos que

$$
\begin{aligned}
& \frac{d x}{d s}=\frac{\cos \sigma}{\sqrt{E}} \\
& \frac{d y}{d s}=\frac{\sin \sigma}{\sqrt{G}} .
\end{aligned}
$$

Na outra mão, $\xi=-\sqrt{\frac{G}{E}} \frac{d y}{d s} \frac{\partial}{\partial x}+\sqrt{\frac{E}{G}} \frac{d x}{d s} \frac{\partial}{\partial y}$ e $\kappa_{g}=\frac{1}{2 \sqrt{E G}}\left\{\frac{\partial G}{\partial x} \frac{d y}{d s}-\frac{\partial E}{\partial y} \frac{d x}{d s}\right\}+\frac{d \sigma}{d s}$. Assim substituindo estas expressões em (1.7) temos que

$$
\frac{d \sigma}{d s}=\frac{\partial}{\partial y}\left(\ln \sqrt{E \mathscr{V}^{2}}\right) \frac{\cos \sigma}{\sqrt{G}}-\frac{\partial}{\partial x}\left(\ln \sqrt{G^{2}}\right) \frac{\sin \sigma}{\sqrt{E}}+\mathbf{h}
$$

Em todos os exemplos estudados neste trabalho temos sempre coordenadas deste tipo. Portanto, a análise da equação (1.7) é equivalente à do sistema seguinte (em cada caso):

$$
\begin{aligned}
& \frac{d x}{d s}=\frac{\cos \sigma}{\sqrt{E}} \\
& \frac{d y}{d s}=\frac{\sin \sigma}{\sqrt{G}} \\
& \frac{d \sigma}{d s}=\frac{\partial}{\partial y}\left(\ln \sqrt{E \mathscr{V}^{2}}\right) \frac{\cos \sigma}{\sqrt{G}}-\frac{\partial}{\partial x}\left(\ln \sqrt{G^{\mathscr{V}^{2}}}\right) \frac{\sin \sigma}{\sqrt{E}}+\mathbf{h}
\end{aligned}
$$

(iii) Note que, (1.7) é uma equação diferencial ordinária definida na superfície $\tilde{M}_{P}$. Esta é singular nos estratos singulares de $\tilde{M}$ pois $\mathscr{V}$ anula-se neste conjunto (vide Proposição 1.11). Porém, podemos considerar soluções de (1.7) saindo ortogonalmente de um estrato de codimensão um (vide Lema 0.6). Nesse caso a hipersuperfície gerada é não singular. 


\subsection{Ações polares}

Dizemos que o par $(H, M)$ é polar (ou que a ação de $H$ sobre $M$ é polar) se existe uma subvariedade conexa e completa $\Sigma$ de $M$ que intersecta todas as $H$-órbitas ortogonalmente. Nesse caso dizemos que $\Sigma$ é uma seção para o par $(H, M)$. Se existe uma seção flat (em relação à métrica induzida) dizemos que $(H, M)$ é hiperpolar (ou que a ação de $H$ sobre $M$ é hiperpolar). Em particular, todo par hiperpolar é polar.

Lema 1.20 (Vide [PT88],[Gor10]). Seja $(H, M)$ um par polar. Então:

(i) Toda seção de $(H, M)$ é totalmente geodésica.

(ii) Para todo $x \in M$ existe uma seção $\Sigma_{x}$ passando por $x$.

Proposição 1.21 (Vide [PT88],[Gor10]). Se (H,M) é polar então a ação slice é polar para cada ponto de $M$. Ainda mais, $T_{x} \Sigma$ é uma seção de $\left(H_{x}, \nu_{x} H(x)\right)$ se $\Sigma$ é uma seção de $(H, M)$ passando por $x \in M$. Em particular, $H_{x}$ atua transitivamente sobre o conjunto de todas as seções passando por $x \in M$.

Definição 1.22. Seja $(H, M)$ um par polar que admita uma seção fechada $\Sigma$. O normalizador $\mathscr{N}(\Sigma)$ e o centralizador $\mathscr{Z}(\Sigma)$ de $\Sigma$ em relação a $H$ são dados por

$$
\mathscr{N}(\Sigma)=\{h \in H: h(\Sigma)=\Sigma\}
$$

$\mathrm{e}$

$$
\mathscr{Z}(\Sigma)=\{h \in H: h(\sigma)=\sigma, \text { para todo } \sigma \in \Sigma\}
$$

O grupo de Weyl generalizado para o par polar $(H, M)$ em relação à seção fechada $\Sigma$, é o quociente

$$
W_{\Sigma}=\mathscr{N}(\Sigma) / \mathscr{Z}(\Sigma)
$$

Temos que $W_{\Sigma}$ é um grupo discreto que age naturalmente sobre $\Sigma$. Seja $\Sigma$ uma seção mergulhada. Então o orbifold $\Delta=W_{\Sigma} \backslash \Sigma$ é homeomorfo (e isométrico como espaço métrico) ao espaço das $H$-órbitas $M=H \backslash \tilde{M}$. Com efeito, a aplicação $\phi: \Delta \rightarrow \tilde{M}, W_{\Sigma}(x) \mapsto H(x)$ está bem definida e, como $\Sigma$ encontra todas as $H$-órbitas, é sobrejetora. Agora sejam $x, y \in \Sigma$ tais que $y=h(x)$ para algum $h \in H$, i.e. $H(x)=H(y)$. Então $\Sigma$ e $h(\Sigma)$ são duas secções passando por $y$. A proposição 1.21 implica que $g(\Sigma)=h(\Sigma)$, para algum $g \in H_{y}$. Logo $g^{-1} h \in \mathscr{N}(\Sigma)$. Isto mostra que $\phi$ é injetora. Na outra mão, o fato de $\Sigma$ ser uma seção implica que $\phi$ é uma isometria. 


\section{CAPÍTULO 2}

\section{Hipersuperfícies mínimas de espaços simétricos não compactos}

Para usar concretamente o método da geometria diferencial equivariante, necessitamos de variedades ambiente cujos grupos de isometrias sejam conhecidos, para assim escolher subgrupos que atuem com cohomogeneidade dois. No caso dos espaços simétricos do tipo não compacto, a decomposição de Iwasawa da componente conexa da identidade do grupo de isometrias nos dá uma maneira natural de procurar por esses subgrupos.

Este capítulo está dividido em três seções. Na primeira apresentamos a decomposição de Iwasawa. Na segunda construímos hipersuperfícies mínimas de espaços simétricos de posto maior do que um. Na última seção lembramos os conceitos básicos da geometria do espaço hiperbólico quaterniônico $\mathbf{H}_{\mathbf{Q}}^{n}$, assim como a construção de seus diferentes modelos. Introduzimos certo tipo de hipersuperfícies mínimas chamadas fans. Estes objetos foram introduzidos para geometria hiperbólica complexa em [GP92]. Finalmente, construímos hipersuperfícies mínimas de $\mathbf{H}_{\mathbf{Q}}^{n}$.

\subsection{Decomposição de Iwasawa}

Para uma prova dos fatos descritos nesta seção, assim como para um estudo mais profundo dos assuntos discutidos, o leitor pode consultar [Kna96], [Hel78] ou [BCO03].

Sejam $G$ um grupo de Lie não compacto, conexo e semisimples, $\mathfrak{G}$ sua álgebra de Lie e $B$ sua forma de Killing. Seja $\theta$ uma involução de Cartan sobre $\mathfrak{G}$ e denotemos com $\mathfrak{K}$ e $\mathfrak{M}$ os auto-espaços associados aos auto-valores 1 e -1 de $\theta$ respectivamente. Temos a seguinte decomposição de Cartan

$$
\mathfrak{G}=\mathfrak{K}+\mathfrak{M}
$$

Temos que $B$ é negativa definida sobre $\mathfrak{K}$ e positiva definida sobre $\mathfrak{M}$. Consideremos o produto interno Ad-invariante $\langle\cdot, \cdot\rangle=:-B(\cdot, \theta)$ definido sobre $\mathfrak{G}$. Então a decomposição 
acima é ortogonal em relação a $B$ e $\langle\cdot, \cdot\rangle$. Também

$$
[\mathfrak{K}, \mathfrak{K}] \subset \mathfrak{K}, \quad[\mathfrak{K}, \mathfrak{M}] \subset \mathfrak{M}, \quad[\mathfrak{M}, \mathfrak{M}] \subset \mathfrak{K} .
$$

Seja $K$ o grupo de Lie conexo com álgebra de Lie $\mathfrak{K}$. Então existe uma única involução $\sigma$ de $G$ tal que $\theta=d \sigma$ e $K$ é o conjunto dos pontos fixos de $\sigma$. O centro $Z$ de $G$ está contido em $K$, e $K$ é compacto se e somente se $Z$ é finito. Neste caso $K$ é um subgrupo compacto maximal de $G$.

Seja $\mathfrak{A}$ um subespaço abeliano maximal de $\mathfrak{M}$. Então $\left\{\operatorname{ad}_{H}: H \in \mathfrak{A}\right\}$ é uma família comutativa de endomorfismos auto-adjuntos de $\mathfrak{G}$. Assim existe um conjunto finito de funcionais lineares não nulos $\Lambda$, chamados raízes restritas de $\mathfrak{G}$, tais que

$$
\mathfrak{G}=\mathfrak{G}_{0}+\sum_{\lambda \in \Lambda} \mathfrak{G}_{\lambda}
$$

é uma decomposição $\langle\cdot, \cdot\rangle$ - ortogonal de $\mathfrak{G}$ em auto-espaços, a saber

$$
\mathfrak{G}_{\lambda}=\left\{X \in \mathfrak{G}: \operatorname{ad}_{H} X=\lambda(H) X \text { para todo } H \in \mathfrak{A}\right\}
$$

onde $\lambda=0$ ou $\lambda \in \Lambda$. A multiplicidade $m(\lambda)$ da raiz $\lambda$ é definida como sendo a dimensão do auto-espaço associado. Denotamos com $H_{\lambda}$ o vetor dual do funcional linear $\lambda$ em relação a $\langle\cdot, \cdot\rangle$. Temos as seguintes propriedades

(i) $\left[\mathfrak{G}_{\lambda}, \mathfrak{G}_{\mu}\right] \subset \mathfrak{G}_{\lambda+\mu}$, onde $\mathfrak{G}_{\lambda+\mu}=0$ se $\lambda+\mu \notin \Lambda$

(ii) se $\lambda \in \Lambda$ então $-\lambda \in \Lambda$ e ainda $\theta\left(\mathfrak{G}_{\lambda}\right)=\mathfrak{G}_{-\lambda}$

(iii) $\mathfrak{G}_{0}=\mathfrak{A}+\mathfrak{K}^{\mathfrak{A}}$,

onde $\mathfrak{K}^{\mathfrak{A}}=\{X \in \mathfrak{K}:[X, H]=0$ para todo $H \in \mathfrak{A}\}$ é o centralizador de $\mathfrak{A}$ em $\mathfrak{K}$. Seja $\Lambda^{+} \subset \Lambda$ tal que $\Lambda$ é a união disjunta de $\Lambda^{+}$e $\Lambda^{-}=\left\{-\lambda: \lambda \in \Lambda^{+}\right\}$. Dizemos que $\Lambda^{+}$é um conjunto de raízes (restritas) positivas. Sejam $\mathfrak{K}_{\lambda}=\left(\mathfrak{G}_{\lambda}+\mathfrak{G}_{-\lambda}\right) \cap \mathfrak{K}_{\mathrm{e}} \mathfrak{M}_{\lambda}=\left(\mathfrak{G}_{\lambda}+\mathfrak{G}_{-\lambda}\right) \cap \mathfrak{M}$, para todo $\lambda \in \Lambda$. Então

$$
\mathfrak{K}=\mathfrak{K}^{\mathfrak{A}}+\sum_{\lambda \in \Lambda^{+}} \mathfrak{K}_{\lambda} \text { e } \mathfrak{M}=\mathfrak{A}+\sum_{\lambda \in \Lambda^{+}} \mathfrak{M}_{\lambda}
$$

Seja $\mathfrak{N}=\sum_{\lambda \in \Lambda^{+}} \mathfrak{G}_{\lambda}$, então $\mathfrak{N}$ é uma subálgebra nilpotente de $\mathfrak{G}$. A decomposição em soma direta de subespaços

$$
\mathfrak{G}=\mathfrak{N}+\mathfrak{A}+\mathfrak{K}
$$

é chamada uma decomposição de Iwasawa de $\mathfrak{G} . \mathfrak{S}=\mathfrak{N}+\mathfrak{A}$ é uma subálgebra de Lie solúvel de $\mathfrak{G}$ tal que $[\mathfrak{S}, \mathfrak{S}]=\mathfrak{N}$.

Sejam $N$ e $A$ os subgrupos de Lie conexos de $G$ com álgebras de Lie $\mathfrak{N}$ e $\mathfrak{A}$ respectivamente. Então $G=N A K$ (isto é, para cada $g \in G$ existem únicos $n \in N, a \in A$ e $k \in K$ tais 
que $g=n a k$ ) é dita uma decomposição de Iwasawa de $G$. Temos que $N A$ é um subgrupo solúvel de $G$.

\subsection{Posto maior do que um}

Nesta seção usamos a notação introduzida em $§ 2.1$ com uma exceção: representamos os elementos de $\mathfrak{A}$ com a letra $\xi$. Denotamos com $p$ o posto de $G / K$ (i.e. $p=\operatorname{dim} A$ ) e $\operatorname{com} q$ a dimensão de $G / K$. Supomos sempre que $p>1$.

\subsubsection{Hipersuperfícies mínimas de $G / K$ dadas por hiperplanos de $\mathfrak{A}$}

Sejam $\pi: G \rightarrow G / K$ a projeção canônica e consideremos as translações à esquerda $l_{g}$ sobre $G / K$ dadas pela equação

$$
\pi \circ L_{g}=l_{g} \circ \pi
$$

onde $L_{g}$ é a translação à esquerda sobre $G$, para todo $g \in G$.

Temos que $\phi=\left.\pi\right|_{N A}$ é um difeomorfismo do grupo de Lie $N A$ sobre o espaço simétrico naõ compacto $G / K$. Com efeito, se $n, m \in N$ e $a, b \in A \operatorname{com} \pi(n a)=\pi(m b)$ então $n a k=m b$ para algum $k \in K$, isto acontece se e somente se $n=m, a=b$ and $k=1$. Por outro lado $d \phi_{g} d\left(L_{g}\right) X=d\left(l_{g}\right)_{o} d \pi X$, para todo $g \in N A$ e cada $X \in \mathfrak{N}+\mathfrak{A}$, onde $o=\pi(1)$.

Consideremos a projeção ortogonal $\rho: \mathfrak{G} \rightarrow \mathfrak{M}$. Então a restrição $\left.\rho\right|_{\mathfrak{N}+\mathfrak{A}}$ é um isomorfismo sobre $\mathfrak{M}$. Definimos a métrica invariante à esquerda $(\cdot, \cdot)$ sobre $N A$ por

$$
(X, Y)=\langle\rho X, \rho Y\rangle
$$

para todo par $X, Y \in \mathfrak{N}+\mathfrak{A}$. Portanto se $X_{1}, X_{2} \in \mathfrak{N}$ e $\xi_{1}, \xi_{2} \in \mathfrak{A}$ temos que

$$
\left(X_{1}+\xi_{1}, X_{2}+\xi_{2}\right)=\frac{1}{2}\left\langle X_{1}, X_{2}\right\rangle+\left\langle\xi_{1}, \xi_{2}\right\rangle \text {. }
$$

Então com a métrica acima $\phi: N A \rightarrow G / K$ é uma isometria. Pela fórmula de Koszul, a conexão de Levi-Civita $\nabla$ de $N A$ em relação a $(\cdot, \cdot)$ está dada por

$$
2\left(\nabla_{X} Y, Z\right)=([X, Y], Z)+([Z, X], Y)+([Z, Y], X)
$$

Observação 2.1. Note que a restrição $\left.\exp \right|_{\mathfrak{A}}: \mathfrak{A} \rightarrow A$ é uma isometria.

Seja $\delta \in \mathfrak{A}^{*}$ dado por

$$
\delta=\frac{1}{2} \sum_{\lambda \in \Lambda^{+}} m(\lambda) \lambda,
$$

i.e., $\delta$ é a metade da soma (incluindo as multiplicidades) das raízes positivas de $G / K$ (vide 
$\S 2.1)$. Seja $\xi_{*} \in \mathfrak{A}$ o vetor dual de $\delta$. Fixemos um vector unitário $\xi_{0} \in \mathfrak{A}$ e sejam $\mathfrak{A}_{0}=\xi_{0}^{\perp}$ o complemento ortogonal de $\mathbf{R} \xi_{0}$ em $\mathfrak{A}$ e $A_{0}=\exp \left(\mathfrak{A}_{0}\right)$. Consideremos a hipersuperfície $\left.\phi\right|_{N A_{0}}: N A_{0} \rightarrow F_{0}=\phi\left(N A_{0}\right)$ de $G / K$. O espaço tangente a $F_{0}$ em $o$ é $\mathfrak{N}+\mathfrak{A}_{0}$ e a segunda forma fundamental $I I$ de $F_{0}$ em $o$ é dada por

$$
\left(I I(X, Y), \xi_{0}\right)=\left(\nabla_{X} Y, \xi_{0}\right)
$$

para todo par $X, Y \in \mathfrak{N}+\mathfrak{A}_{0}$. Da equação $(2.1)$ segue que $I I(\xi, \xi)=0$ para cada $\xi \in \mathfrak{A}_{0}$, e

$$
\left(I I(X, X), \xi_{0}\right)=\lambda\left(\xi_{0}\right)(X, X)
$$

para todo $X \in \mathfrak{G}_{\lambda}, \lambda \in \Lambda^{+}$. Logo em o temos que

$$
\left(\operatorname{tr}(I I), \xi_{0}\right)=\sum_{\lambda \in \Lambda^{+}} m(\lambda) \lambda\left(\xi_{0}\right)=2 \delta\left(\xi_{0}\right)=2\left\langle\xi_{*}, \xi_{0}\right\rangle
$$

Portanto, $F_{0}$ tem curvatura média constante igual a $2\left\langle\xi_{*}, \xi_{0}\right\rangle$ e é mínima se e somente se $\xi_{0}$ é ortogonal a $\xi_{*}$.

Sejam $a_{t}=\exp \left(t \xi_{0}\right)$ e $F_{t}=N \exp \left(\mathfrak{A}_{0}+t \xi_{0}\right)$, para todo $t \in \mathbf{R}$. Assim obtemos uma folheação de $G / K$ por hipersuperfícies homogêneas congruentes entre si e de curvatura média constante, a saber $F_{t}=L_{a_{t}}\left(F_{0}\right), t \in \mathbf{R}$.

Observação 2.2. Note que, as folhas da folheação $\mathfrak{F}_{\xi_{0}}=\left(F_{t}\right)_{t \in \mathbf{R}}$ construída acima, são órbitas da ação do grupo $N A_{0}$ que atua sobre $G / K$ com cohomogeneidade um. Em [BCO03], §9.5 e) é feita a classificação das hipersuperfícies homogêneas de espaços simétricos irredutíveis de tipo não compacto. Em particular temos que uma outra folheação $\mathfrak{F}_{\xi_{1}}$, construída como foi feito acima, é congruente a $\mathfrak{F}_{\xi_{0}}$ se e somente se existe um automorfismo do diagrama de Dynkin $^{1}$ relativo às raízes de $G / K$, que leva $\xi_{0}$ em $\xi_{1}$. Ainda mais, toda folheação homogênea de $G / K$ é congruente a uma deste tipo.

\subsubsection{Hipersuperfícies mínimas e equivariantes de $G / K$}

a) $H=N A_{1}, p \geq 2$

Sejam $\mathfrak{A}=\mathfrak{A}_{1}+\mathfrak{A}_{2}$ uma decomposição ortogonal com $\operatorname{dim} \mathfrak{A}_{2}=2$ e $A_{i}=\exp \left(\mathfrak{A}_{i}\right)$, para $i=1,2$. Consideremos o subgrupo fechado $H=N A_{1}$ de $G=\operatorname{Iso}_{0}(G / K)$. Temos que $H$ age livremente sobre $G / K$, $\operatorname{logo}(H, G / K)$ tem um único tipo de órbita (e portanto, principal) que é difeomorfo a $H \approx \mathbf{R}^{q-2}$. Na outra mão $A_{2} \cong \mathfrak{A}_{2}$ (vide Observação 2.1 ) é uma seção para $(H, G / K)$, mais ainda o grupo de Weyl correspondente é trivial, logo

$$
H \backslash G / K=\mathfrak{A}_{2} .
$$

\footnotetext{
${ }^{1}$ Note que podemos considerar $\xi_{0}$ e $\xi_{1}$ pertencendo a uma câmera de Weyl fixada
} 
Calculemos o funcional de volume. Dado $\xi \in \mathfrak{A}_{2}$, se $Y \in \mathfrak{A} \cup \mathfrak{G}_{\lambda}$, então

$$
\operatorname{Ad}_{\exp (-\xi)} Y=\left\{\begin{array}{ll}
Y & , \text { se } Y \in \mathfrak{A} \\
\mathrm{e}^{-\lambda(\xi)} Y & , \text { se } Y \in \mathfrak{G}_{\lambda}
\end{array} .\right.
$$

Seja $a=\exp (\xi) \in A_{2}$. Então, o campo de Killing $Y^{*}(a)$ é dado por

$$
Y^{*}(a)=\left\{\begin{array}{ll}
a_{*} Y^{*}(0) & \text {, se } Y \in \mathfrak{A} \\
\mathrm{e}^{-\lambda(\xi)} a_{*} Y^{*}(0) & , \text { se } Y \in \mathfrak{G}_{\lambda}
\end{array} .\right.
$$

Assim escolhendo bases ortonormais de $\mathfrak{A}_{1}$ e $\mathfrak{G}_{\lambda}$, para cada $\lambda \in \Lambda^{+}$, e calculando o determinante (1.3) obtemos

$$
\mathscr{V}(\xi)=\mathrm{e}^{-\sum_{\lambda \in \Lambda^{+}} m(\lambda) \lambda(\xi)}=\mathrm{e}^{-2\left\langle\xi_{*}, \xi\right\rangle}
$$

para todo $\xi \in \mathfrak{A}_{2}$. A métrica orbital $d s^{2}$ é a restrição $\left.\langle\cdot, \cdot\rangle\right|_{\mathfrak{A}_{2}}$. A métrica modificada é $d \bar{s}^{2}=\mathscr{V}^{2} d s^{2}$. Suponhamos que $\xi_{*} \in \mathfrak{A}_{1}$. Então, de (2.4) segue que $\mathscr{V} \equiv 1$. Portanto, a métrica modificada $d \bar{s}^{2}$ coincide com a métrica orbital $d s^{2}$. Assim $\left(\mathfrak{A}_{2}, d \bar{s}^{2}\right)$ é o espaço euclidiano bidimensional. Segue que as retas em $\mathfrak{A}_{2}$ geram subvariedades mínimas em $G / K$. Estas correspondem às hipersuperfícies construídas em $\S 2.2 .1$.

Daqui em diante $\xi_{*} \notin \mathfrak{A}_{1}$. Seja $\xi_{*}^{\prime} \neq 0$ a projeção ortogonal de $\xi_{*}$ sobre $\mathfrak{A}_{2}$. Note que de (2.4) segue que $\mathscr{V}(\xi)=\mathrm{e}^{-2\left\langle\xi_{*}^{\prime}, \xi\right\rangle}$, para todo $\xi \in \mathfrak{A}_{2}$ Dada uma reta $\ell \subset \mathfrak{A}_{2}$, paralela a $\xi_{*}^{\prime}$, a reflexão de $\mathfrak{A}_{2}$ em relação a $\ell$ é uma isometria de $\mathfrak{A}_{2}$ que fixa $\mathscr{V}$, portanto é uma isometria de $\left(\mathfrak{A}_{2}, d \bar{s}^{2}\right)$. Temos que $\ell$ é o conjunto dos pontos fixos de dita reflexão, assim $\ell$ é uma geodésica de $\left(\mathfrak{A}_{2}, d \bar{s}^{2}\right)$. Estas geodésicas geram o mesmo tipo de hipersuperfícies construídas em $\S 2.2 .1$.

Temos que $\left(\mathfrak{A}_{2}, d \bar{s}^{2}\right)$ é conforme ao plano euclidiano. Consideremos coordenadas $(x, y)$ tais que $d s^{2}=d x^{2}+d y^{2}$ e $\mathscr{V}(x, y)=\mathrm{e}^{-B y}$, onde $B=2\left|\xi_{*}^{\prime}\right|$. Logo, do Teorema 1.18 obtemos

Proposição 2.3. Seja $\gamma(s)=(x(s), y(s))$ uma curva em $\mathbf{R}^{2}$ parametrizada pelo comprimento de arco s (em relação à métrica $d s^{2}$ ) e $\sigma$ o ângulo ente $\frac{\partial}{\partial x}$ e $\frac{d \gamma}{d s}$. Então a hipersuperfície $\Gamma$ de $G / K$, com $H \backslash \Gamma=\gamma$ tem curvatura média $\mathbf{h}$ se e somente se

$$
\begin{aligned}
& \frac{d x}{d s}=\cos \sigma \\
& \frac{d y}{d s}=\sin \sigma \\
& \frac{d \sigma}{d s}=-B \cos \sigma+\mathbf{h} .
\end{aligned}
$$

Observação 2.4. Soluções explícitas. Note que, se $\mathbf{h} \equiv B \cos c$, então a reta $y=y_{0}+(x-$ $\left.x_{0}\right) \tan c$ é uma solução de 2.5, que gera uma hipersuperfície de curvatura média constante. Em particular, as retas paralelas ao eixo y (i.e. as retas em $\mathfrak{A}_{2}$ paralelas a $\xi_{*}^{\prime}$ ) correspondem às soluções $\sigma= \pm \frac{\pi}{2}$, que por sua vez geram hipersuperfícies mínimas. 


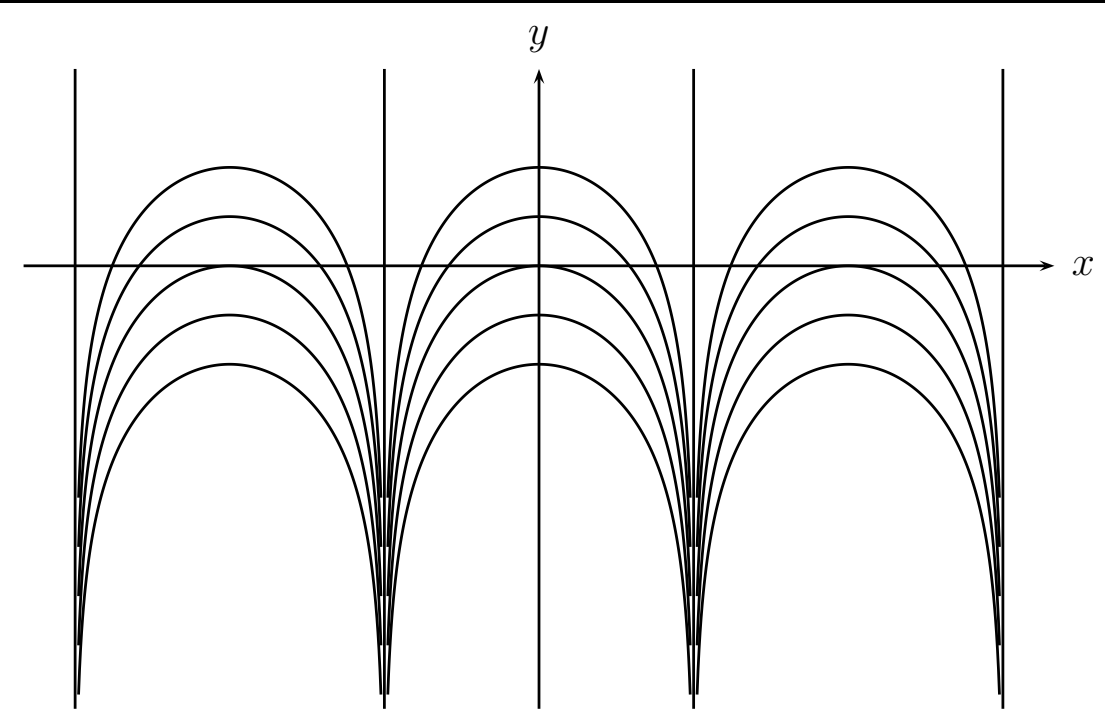

Figura 2.1: Soluções de (2.5)

Note que as soluções explícitas geram o mesmo tipo de hipersuperfícies construídas em §2.2.1. Em particular são hipersuperfícies homogêneas.

Demonstração do Teorema 0.1(i). Com efeito, consideremos soluções de (2.5) para $\mathbf{h} \equiv 0$. Temos exatamente dois tipos de soluções.

Da observação 2.4 as retas paralelas ao eixo $y$ (que evidentemente folheiam $\mathbf{R}^{2}$ ) correspondem a soluções de (2.5) que geram uma folheação de $G / K$ com folhas homogêneas. Estas folhas são difeomorfas a $H \times \mathbf{R} \approx \mathbf{R}^{q-1}$. Na outra mão, as translações ao longo do eixo $x$ induzem transvecções em $G / K$ que (geram) deixam invariante tal folheação. Isto mostra o item (a).

Por unicidade, a solução de (2.5), passando por $\left(x_{0}, y_{0}, \pm \frac{\pi}{2}\right)$ corresponde à reta $x=x_{0}$. Então, para soluções diferentes destas podemos considerar $\sigma \in\left(-\frac{\pi}{2}, \frac{\pi}{2}\right)$. Assim, integrando (2.5), usando $\sigma$ com parâmetro, obtemos soluções da forma $x=x_{0}-\frac{1}{B}\left(\sigma-\sigma_{0}\right)$ e $y=$ $y_{0}+\frac{1}{B} \ln \left(\frac{\cos \sigma}{\cos \sigma_{0}}\right)$. Por outro lado, o sistema (2.5) é invariante pelas translações $(x, y) \mapsto$ $\left(x+x_{0}, y+y_{0}\right)$. Logo, a curva $\gamma_{0}$ dada por $y=\frac{1}{B} \ln (\cos B x), \operatorname{com} x \in\left(-\frac{\pi}{2 B}, \frac{\pi}{2 B}\right)$, i.e. a projeção no plano $(x, y)$ da solução de $(2.5)$ com valores iniciais $\left(x_{0}, y_{0}, \sigma_{0}\right)=(0,0,0)$, gera as demais soluções mediante translações. Em particular, dado $x_{0} \in \mathbf{R}$, a faixa entre as retas $x=x_{0}$ e $x=x_{0}+\frac{\pi}{B}$ é folheada por soluções do tipo $\gamma_{0}$. Sejam $F_{0}$ e $F_{1}$ as hipersuperfícies geradas por tais retas. Então, a faixa aberta entre $F_{0}$ e $F_{1}$ é folheada por hipersuperfícies assintóticas a estas, difeomorfas a $H \times \gamma_{0} \approx \mathbf{R}^{q-1}$. Esta folheação é invariante por um grupo a um parâmetro de transvecções (induzidas por translações ao longo do eixo y). Para finalizar, note que $\left|\xi_{*}\right|=|\delta|$ (vide equação (2.2)). Logo dado $d \geq \frac{\pi}{2|\delta|}$, podemos escolher $\mathfrak{A}_{2}$ de maneira tal que $d=\frac{\pi}{B}=\frac{\pi}{2\left|\xi_{*}^{\prime}\right|}$. Isto conclui a demonstração do item (b). 


\section{b) $H=N, p \geq 3$ e dupla redução}

Em diante vamos supor que $p \geqq 3$. Consideremos a ação à esquerda de $N$ sobre $G / K$ dada pelas translações à esquerda por elementos de $N$. Esta ação é livre, por tanto temos um único tipo de órbita de $(N, G / K)$ difeomorfa a $N \approx \mathbf{R}^{q-p} . A \cong \mathfrak{A}$ (vide Observação 2.1) é uma seção para $(N, G / K)$ com respectivo grupo de Weyl trivial, assim

$$
N \backslash G / K=\mathfrak{A} .
$$

A métrica orbital $d s^{2}$ é a restrição $\left.\langle\cdot, \cdot\rangle\right|_{\mathfrak{A}}$. O funcional de volume (vide o caso a) acima) é dado por

$$
\mathscr{V}(\xi)=\mathrm{e}^{-2\left\langle\xi_{*}, \xi\right\rangle}
$$

para todo $\xi \in \mathfrak{A}$.

Observação 2.5. Note que $\operatorname{Iso}(\mathfrak{A})=O(\mathfrak{A}) \ltimes \mathfrak{A}$ (isometrias intrínsecas). Na outra mão as translações $H \mapsto H^{\prime}+H, H \in \mathfrak{A}$ correspondem às translações à esquerda $l_{\exp \left(H^{\prime}\right)}$, que por sua vez são isometrias de $G / K$ (isometrias extrínsecas de $\mathfrak{A}$ em $G / K$ ). No entanto, temos somente um subgrupo finito de $O(\mathfrak{A})$ que provém de $G=\operatorname{Iso}_{0}(G / K)$, a saber o grupo de Weyl.

As aplicações ortogonais de $\mathfrak{A}$ que fixam $\xi_{*}$ deixam $\mathscr{V}$ invariante. Logo são isometrias de $\left(\mathfrak{A}, d \bar{s}^{2}\right)$ representadas por $O(p-1)$. Usaremos de novo o método da geometria diferencial equivariante para construir hipersuperfícies mínimas de $\overline{\mathfrak{A}}=\left(\mathfrak{A}, d \bar{s}^{2}\right)$ invariantes por $S O(p-$ 1). Ou seja, construiremos hipersuperfícies de $G / K$ com cohomogeneidade $p-1$. Neste caso a métrica modificada é $d \bar{s}^{2}=\mathscr{V}^{\frac{2}{p-1}} d s^{2}$. De fato, as isometrias de $\overline{\mathfrak{A}}$ representadas por $O(p-1)$ são intrínsecas, logo a segunda redução é necessária. Vide Observação 2.5.

Temos que $\overline{\mathfrak{A}}$ é conforme ao espaço euclidiano $p$-dimensional $\mathbf{E}^{p}$. Sejam $\left(u_{1}, \ldots, u_{p}\right)$ coordenadas tais que $d s^{2}=d u_{1}^{2}+\cdots+d u_{p}^{2}$ e $\mathscr{V}\left(u_{1}, \ldots, u_{p}\right)=\mathrm{e}^{-B u_{p}}$, onde $B=2\left|\xi_{*}\right|$. Seja $\bar{H}=S O(p-1)$ o subgrupo de isometrias de que fixa $\frac{\partial}{\partial u_{p}}$. Assim, os tipos de isotropia e de órbita de $(\bar{H}, \overline{\mathfrak{A}})$ são

$$
\bar{H}_{u}= \begin{cases}S O(p-1), & \text { se } u_{1}=\cdots=u_{p-1}=0 \\ S O(p-2), & \text { caso contrário }\end{cases}
$$

e

$$
\bar{H} / \bar{H}_{u} \approx \begin{cases}\text { um ponto, } & \text { se } u_{1}=\cdots=u_{p-1}=0 \\ \mathbf{S}^{p-2}, & \text { caso contrário }\end{cases}
$$

respectivamente.

Observação 2.6. Note que o par $\left(S O(p-1), \mathbf{E}^{p}\right)$ é polar, assim, como $\overline{\mathfrak{A}}$ é conforme a $\mathbf{E}^{p}$, temos que $(\bar{H}, \overline{\mathfrak{A}})$ também é polar. Portanto a métrica orbital é dada pela restrição de $d \bar{s}^{2}$ a uma seção dada. Então, denotaremos com $d \bar{s}^{2}$ a métrica orbital. 
Na outra mão, calculemos o volume $\overline{\mathscr{V}}$ das órbitas de $(\bar{H}, \overline{\mathfrak{A}})$ como descrito em $\S 1.3$. Seja $\left\{X_{1}, \ldots, X_{p-2}\right\}$ uma base ortonormal de $\overline{\mathfrak{H}}^{\perp}=\mathfrak{S O}(p-2)^{\perp} \subset \mathfrak{S O}(p-1)$. Então

$$
\begin{aligned}
\overline{\mathscr{V}}(u)^{2} & =\operatorname{det}\left(d \bar{s}^{2}\left(X_{i}^{*}(u), X_{j}^{*}(u)\right)\right) \\
& =\operatorname{det}\left(\mathscr{V}(u)^{\frac{2}{p-1}} d s^{2}\left(X_{i}^{*}(u), X_{j}^{*}(u)\right)\right) \\
& =\mathscr{V}(u)^{\frac{2(p-2)}{p-1}} \operatorname{det}\left(d s^{2}\left(X_{i}^{*}(u), X_{j}^{*}(u)\right)\right) \\
& =\mathscr{V}(u)^{\frac{2(p-2)}{p-1}}\left(u_{1}^{2}+\cdots+u_{p-1}^{2}\right)^{p-2} \\
& =\mathrm{e}^{-\frac{2 B(p-2) u_{p}}{p-1}}\left(u_{1}^{2}+\cdots+u_{p-1}^{2}\right)^{p-2} .
\end{aligned}
$$

$\Sigma=\left\{u \in \mathbf{R}^{p}: u_{1}=\cdots=u_{p-2}=0\right\}$ é uma seção para $(\bar{H}, \overline{\mathfrak{A}})$. O grupo de Weyl $W_{\Sigma}$ é $\mathbf{Z}_{2}$ e temos que o espaço das órbitas $\bar{H} \backslash \overline{\mathfrak{A}}$ é o semi-plano

$$
\Delta=\left\{(x, y) \in \mathbf{R}^{2}: y \geq 0\right\}
$$

A projeção canônica $\pi: \mathbf{R}^{p} \rightarrow \Delta$ é dada por $\pi(u)=(x, y)$, onde

$$
x=u_{p} \text { e } y=\sqrt{u_{1}^{2}+\cdots+u_{p-1}^{2}} \text {. }
$$

A métrica orbital e o funcional de volume no ponto $(x, y) \in \Delta$ (vide Observação 2.6) são dados por

$$
\begin{aligned}
d \bar{s}^{2} & =\mathrm{e}^{-\frac{2 B x}{p-1}}\left(d x^{2}+d y^{2}\right) \\
\overline{\mathscr{V}}(x, y) & =\mathrm{e}^{-\frac{B(p-2) x}{p-1}} y^{p-2} .
\end{aligned}
$$

Logo a métrica (pela segunda vez) modificada é

$$
\begin{aligned}
d \overline{\bar{s}}^{2} & =\overline{\mathscr{V}}^{2} d \bar{s}^{2} \\
& =\mathrm{e}^{-\frac{2 B(p-2) x}{p-1}} y^{2 p-4} \mathrm{e}^{-\frac{2 B x}{p-1}}\left(d x^{2}+d y^{2}\right) \\
& =\mathrm{e}^{-2 B x} y^{2 p-4}\left(d x^{2}+d y^{2}\right) \\
& =\mathscr{W}^{2} d s^{2}
\end{aligned}
$$

onde $\mathscr{W}(x, y)=\mathrm{e}^{-B x} y^{p-2}$ e $d s^{2}=d x^{2}+d y^{2}$. Portanto, o Teorema 1.18 implica

Proposição 2.7. Seja $\gamma(s)=(x(s), y(s))$ uma curva em $\Delta$, parametrizada pelo comprimento de arco s (em relação à métrica $d s^{2}$ ) e $\sigma$ o ângulo ente $\frac{\partial}{\partial x}$ e $\frac{d \gamma}{d s}$. Então a hipersuperfície 
$\Gamma$ de $G / K$, com $N \backslash \Gamma=\bar{\Gamma}$ e $\bar{H} \backslash \bar{\Gamma}=\gamma$ é mínima se e somente se

$$
\begin{aligned}
& \frac{d x}{d s}=\cos \sigma \\
& \frac{d y}{d s}=\sin \sigma \\
& \frac{d \sigma}{d s}=(p-2) \frac{\cos \sigma}{y}+B \sin \sigma .
\end{aligned}
$$

Demonstração. Com efeito, do Teorema 1.18 segue que $\bar{\Gamma}$ é mínima em $\overline{\mathfrak{A}}$ se e somente se $\gamma$ é uma geodésica em relação à métrica modificada $d \bar{s}^{2}$. Na outra mão, como $d \bar{s}^{2}=\mathscr{W} d s^{2}$, então do Lema (1.16) segue que

$$
\mathscr{W}^{2} \overline{\bar{\kappa}}_{g}=\kappa_{g}-\frac{d}{d \xi} \ln \mathscr{W}
$$

onde $\kappa_{g}$ e $\overline{\bar{\kappa}}_{g}$ são as curvaturas geodésicas de $\gamma$ em relação às métricas $d s^{2}$ e $d \bar{s}^{2}$, respectivamente; e $\xi$ é o campo normal unitário, na métrica $d s^{2}$, ao longo $\gamma$. Assim $\gamma$ é uma geodésica em relação a $d \overline{\bar{s}}^{2}$ se somente se $\kappa_{g}-\frac{d}{d \xi} \ln \mathscr{W}=0$. Esta última equação é equivalente a (2.6) (vide Observação 1.19(ii)). Na outra mão, pela Proposição $1.17 \bar{\Gamma}$ é mínima em $\overline{\mathfrak{A}}$ se e somente se $\Gamma$ é mínima em $G / K$.

Observação 2.8. (i) Note que o sistema (2.6) é singular no bordo $\partial \Delta=\left\{(x, y) \in \mathbf{R}^{2}\right.$ : $y=0\}$. No entanto podemos considerar soluções saindo de $\partial \Delta$ com ângulo reto. Vide Lema 0.6 .

(ii) O sistema (2.6) é invariante pelas trasformações conformes

$$
(x, y, \sigma) \mapsto(x+t, y, \sigma)
$$

Assim, basta considerar a solução com valores iniciais $\left(0,0, \frac{\pi}{2}\right)$.

Lema 2.9. Seja $c(s)=(x(s), y(s), \sigma(s))$ a solução de (2.6) con valores iniciais $c(0)=$ $\left(0,0, \frac{\pi}{2}\right)$. Então

(i) $\frac{d \sigma}{d s}(0)=\frac{B}{p-1}>0$

(ii) $\sigma(s) \in\left(\frac{\pi}{2}, \pi\right)$ se $s>0$

(iii) $\lim _{s \rightarrow+\infty} y(s)=+\infty$

(iv) $\lim _{s \rightarrow+\infty} \sigma(s)=\pi$

(v) $\lim _{s \rightarrow+\infty} x(s)=-\infty$ 
Demonstração. O item (i) segue facilmente de multiplicar a terceira equação em (2.6) por $y$, diferenciar e computar em $s=0$.

Note que da terceira equação em (2.6) segue que

$$
\frac{d \sigma}{d s}(s)=\left\{\begin{array}{ll}
B>0 & , \text { se } \sigma(s)=\frac{\pi}{2} \\
-\frac{p-2}{y(s)}<0 & , \text { se } \sigma(s)=\pi
\end{array},\right.
$$

onde $s>0$. Isto junto com (i) mostra (ii).

Consideremos a função

$$
I=\left(\cos \sigma+\frac{B}{(p-2)} y \sin \sigma\right) \mathrm{e}^{-B x} y^{p-3}
$$

Diferenciando $I$ ao longo de $c$ temos que $\frac{d I}{d s}=-\frac{1}{2} \mathrm{e}^{-2 B x} y^{p-4} \sin 2 \sigma$. Assim, por (ii), $\frac{d I}{d s}(s)>0$ para $s>0$. Como $I(0)=0$, então $I(s)>0$, se $s>0$. Portanto

$$
\tan \sigma \leqq-\frac{(p-2)}{B y}
$$

De (ii) e da segunda equação em (2.6) segue que $y$ cresce monotonicamente. Suponhamos que $\lim _{s \rightarrow+\infty} y(s)=Y$, para algum $Y>0$, então, de novo usando a equação para $y$ em (2.6) obtemos $\lim _{s \rightarrow+\infty} \sigma(s)=\pi$. Mas isso não é possível por (2.7). Logo segue o item (iii).

Agora consideremos a função

$$
J=\left(\cos \sigma+\frac{B}{p-1} y \sin \sigma\right) \mathrm{e}^{-\frac{p-2}{p-1} B x} y^{p-2} .
$$

Diferenciando $J$ ao longo de $c$ temos que $\frac{d J}{d s}=\frac{B^{2}}{2(p-1)^{2}} \mathrm{e}^{-\frac{p-2}{p-1} B x} y^{p-1} \sin 2 \sigma$. Assim de (ii) $\frac{d J}{d s}<0$, se $s>0$. Como $J(0)=0$, então $J(s)<0$, se $s>0$. Portanto

$$
-\frac{(p-1)}{B y} \leqq \tan \sigma
$$

Então, combinando (2.7) e (2.8), do item (iii) segue que $\lim _{s \rightarrow+\infty} \tan \sigma(s)=0$. Isto junto com (ii) mostra (iv).

Finalmente, da segunda equação em (2.6) e de (ii) segue que $x$ decresce monotônicamente. Logo (v) segue de (iv).

Proposição 2.10. Para cada $t \in \mathbf{R}$ seja $c_{t}$ a solução de (2.6) com valores iniciais $\left(t, 0, \frac{\pi}{2}\right)$ e seja $\gamma_{t}$ sua projeção em $\Delta$. Então a família a um parâmetro de curvas $\left(\gamma_{t}\right)_{t \in \mathbf{R}}$ é uma folheação de $\Delta$ (vide Figura 2.2).

Demonstração. Segue diretamente da Observação 2.8(ii) e do Lema 2.9.

Demonstração do Teorema 0.1(ii). Pela Proposição 2.10, a família $\left(\gamma_{t}\right)_{t \in \mathbf{R}}$ gera uma folheação $\left(\bar{\Gamma}_{t}\right)_{t \in \mathbf{R}}$ de $\overline{\mathfrak{A}}$ por hipersuperfícies mínimas, $\bar{H}$-equivariantes e difeomorfas a $\mathbf{R}^{p-1}$. 


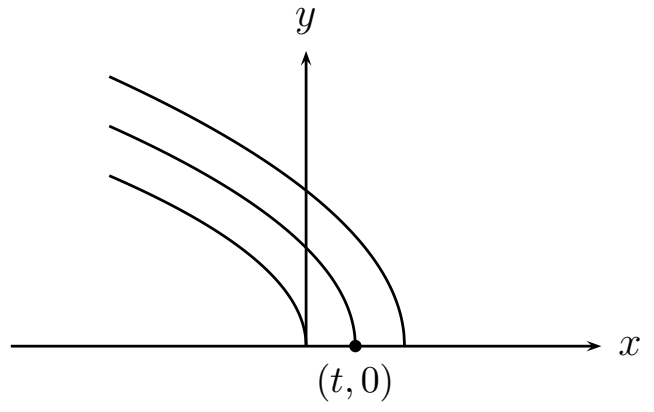

Figura 2.2: Soluções de (2.6) saindo ortogonalmente da fronteira $y=0$

Esta por sua vez gera uma folheação $\left(\Gamma_{t}\right)_{t \in \mathbf{R}}$ de $G / K$ por hipersuperfícies mínimas $N$ equivariantes e difeomorfas a $N \times \mathbf{R}^{p-1} \approx \mathbf{R}^{q-1}$. Finalmente, note que as translações na Observação 2.8(ii) induzem transveções em $G / K$ que deixam $\left(\Gamma_{t}\right)_{t \in \mathbf{R}}$ invariante.

\subsection{Posto um: o espaço hiperbólico quaterniônico}

É um fato bem conhecido que os espaços simétricos não compactos de posto um são os espaços hiperbólicos $\mathbf{H}_{\mathbf{K}}^{n}$ (vide por exemplo [Mos73] e [BCO03]), onde $\mathbf{K}$ é uma das seguintes álgebras com divisão $\mathbf{R}, \mathbf{C}, \mathbf{Q}$ e $\mathbf{K}=\mathbf{O}$ se $n=2$ (reais, complexos, quatérnions e octônions, respectivamente). A saber

$$
\begin{aligned}
& \mathbf{H}_{\mathbf{R}}^{n}=S O_{0}(n, 1) / S O(n), \\
& \mathbf{H}_{\mathbf{C}}^{n}=S U(n, 1) / U(n), \\
& \mathbf{H}_{\mathbf{Q}}^{n}=S p(n, 1) / S p(n) \times S p(1), \\
& \mathbf{H}_{\mathbf{O}}^{2}=F_{4}^{-20} / \operatorname{Spin}(9) .
\end{aligned}
$$

Nesta seção construiremos hipersuperfícies mínimas e equivariantes de $\mathbf{H}_{\mathbf{Q}}^{n}$. Para isto, estudaremos subgrupos $H$ de $S p(n, 1)$, adaptados à sua decomposição de Iwasawa, que atuam polarmente sobre $\mathbf{H}_{\mathbf{Q}}^{n}$ com cohomogeneidade dois. A saber, $H$ pode ser do tipo elíptico, loxodrômico ou parabólico. Os casos de $\mathbf{H}_{\mathbf{R}}^{n}$ e $\mathbf{H}_{\mathbf{C}}^{n}$ foram estudados em [dCD83] e [GG00]. O caso de $\mathbf{H}_{\mathbf{O}}^{2}$ escapa do alcance neste momento, mas pretendemos abordá-lo posteriormente. De modo geral, faremos analogia com o estudo feito em [GG00].

Em [GP92] foram introduzidas hipersuperfícies mínimas do espaço hiperbólico complexo chamadas fans. Na presente seção introduzimos este tipo de objeto para o espaço hiperbólico quaterniônico, fazendo analogia com o caso complexo. Fans estão fortemente relacionados com bissetores métricos, os quais foram estudados em [Mos80] e [Gol06]. Mostraremos que esta relação existe também no caso quaterniônico.

As idéias básicas sobre a geometria de $\mathbf{H}_{\mathbf{Q}}^{n}$ foram tomadas de [BGS85],[Gol06], [GP92], [KP03] e [Mos73]. O leitor poderá encontrar mais profundidade nos assuntos aqui tratados nessas referências. 


\subsubsection{Construção de $\mathbf{H}_{\mathrm{Q}}^{n}$}

Seja $\mathbf{Q}$ a álgebra com divisão dos quatérnions. Cada $q \in \mathbf{Q}$ é da forma $q=q_{0}+i q_{1}+j q_{2}+$ $k q_{3}$, onde $q_{0}, q_{1}, q_{2}, q_{3} \in \mathbf{R}$ e $i, j, k$ são elementos tais que $i^{2}=j^{2}=k^{2}=-1$, satisfazendo: $i j=k, j k=i, k i=j$ e $j i=-i j, k j=-j k, i k=-k i$. A involução usual (conjugação) $q \mapsto \bar{q}$ é dada por $\bar{q}=q_{0}-i q_{1}-j q_{2}-k q_{3}$. Denotemos com $|q|, \Re(q)$ e $\Im(q)$, o módulo e as partes real e imaginária de $q$, respectivamente. Lembremos que

$$
\begin{aligned}
\Re(q) & =\frac{1}{2}(q+\bar{q}), \\
\Im(q) & =\frac{1}{2}(q-\bar{q}), \\
|q|^{2} & =q \bar{q}=\bar{q} q, \\
\Im(\mathbf{Q}) & =\{q \in \mathbf{Q}: \Re(q)=0\} .
\end{aligned}
$$

Representemos os elementos do $\mathbf{Q}$-módulo $\mathbf{Q}^{m}$ por vetores coluna $x$ com entradas $x_{1}, \ldots, x_{m}$. Denotemos com $(\cdot, \cdot)$ a forma bilinear hermitiana (positiva definida) canônica em $\mathbf{Q}^{m}$, i.e.

$$
(x, y)=x_{1} \overline{y_{1}}+\cdots+x_{n} \overline{y_{m}},
$$

para todo par $x, y \in \mathbf{Q}^{m}$. Se $x \in \mathbf{Q}^{m}$ então $|x|=\sqrt{(x, x)}$.

Seja $n \geq 1$. Em $\mathbf{Q}^{n+1}$ definimos a forma bilinear hermitiana indefinida $\langle\cdot, \cdot\rangle$ por

$$
\langle x, y\rangle=x_{1} \overline{y_{1}}+\cdots+x_{n} \overline{y_{n}}-x_{n+1} \overline{y_{n+1}},
$$

para cada par $x, y \in \mathbf{Q}^{n+1}$. Consideremos os subconjuntos de $\mathbf{Q}^{n+1}$ seguintes

$$
\begin{gathered}
V_{+}=\left\{x \in \mathbf{Q}^{n+1}:\langle x, x\rangle>0\right\}, \\
V_{0}=\left\{x \in \mathbf{Q}^{n+1}:\langle x, x\rangle=0\right\}, \\
V_{-}=\left\{x \in \mathbf{Q}^{n+1}:\langle x, x\rangle<0\right\},
\end{gathered}
$$

Definimos $\mathbf{H}_{\mathbf{Q}}^{n}$ como sendo a projetivização $\mathbf{P}\left(V_{-}\right)=\left\{[x]: x \in V_{-}\right\}$, i.e o conjunto as retas quaterniônicas em $V_{-}$, equipado com a métrica

$$
d s^{2}=-\langle x, x\rangle^{-2} \operatorname{det}\left[\begin{array}{cc}
\langle x, x\rangle & \langle d x, x\rangle \\
\langle x, d x\rangle & \langle d x, d x\rangle
\end{array}\right] .
$$

A distância $d([x],[y])$ entre dois pontos $[x],[y] \in \mathbf{H}_{\mathbf{Q}}^{n}$ é dada por

$$
d([x],[y])=\operatorname{arccosh} \frac{|\langle x, y\rangle|}{\sqrt{\langle x, x\rangle\langle y, y\rangle}} .
$$


$\mathbf{P}\left(V_{0}\right)=\left\{[x]: x \in V_{0}\right\}$ define a fronteira ideal de $\mathbf{H}_{\mathbf{Q}}^{n}$ que é denotada por $\partial \mathbf{H}_{\mathbf{Q}}^{n}$.

Observação 2.11. Em geral, seja $M$ uma variedade de Hadamard. Dizemos que duas geodésicas $\gamma_{1}, \gamma_{2}: \mathbf{R} \rightarrow M$, são assintóticas se existe uma constante $a \in \mathbf{R}$ tal que $d\left(\gamma_{1}(t), \gamma_{2}(t)\right) \leqq$ $a$, para todo $t \geqq 0$. Isto define uma relação de equivalência sobre o conjunto das geodésicas em $M$. O conjunto das classes de equivalência é denotado por $\partial M$ e é dito fronteira ideal de $M$. Temos que $\partial M$ tem uma estrutura natural de espaço métrico (vide [BGS85]). Dada uma subvariedade mergulhada $N$ de $M$ definimos a sua fronteira ideal $\partial N$ como sendo a intersecção $\bar{N} \cap \partial M$, onde $\bar{N}$ denota o fecho de $N$ relativo a $M \cup \partial M$.

Seja $S p(n, 1)$ o subgrupo de $G L(n+1, \mathbf{Q})$ que preserva $(2.9)$, então $g \in S p(n, 1)$ se e somente se

$$
g I_{n, 1} g^{*}=I_{n, 1},
$$

onde $g^{*}$ denota a matriz transposta conjugada de $g$ e $I_{n, 1}=\left[\begin{array}{cc}1_{n} & 0 \\ 0 & -1\end{array}\right]$, com $1_{n}$ sendo a matriz identidade de ordem $n$. Temos que $S p(n, 1)$ é um grupo de Lie não compacto e semisimples que age naturalmente por isometrias sobre $\mathbf{H}_{\mathbf{Q}}^{n}$. Note que esta ação não é efetiva pois o centro $\mathbf{Z}_{2}=\left\{ \pm 1_{n+1}\right\}$ de $S p(n, 1)$ (matrices escalares reais) age trivialmente. Portanto $\mathbf{P}(S p(n, 1))=\operatorname{Iso}_{0}\left(\mathbf{H}_{\mathbf{Q}}^{n}\right)=S p(n, 1) / \mathbf{Z}_{2}$.

\subsubsection{Decomposição de Iwasawa de $S p(n, 1)$}

Consideremos a reta $o=\left\{x \in V_{-}: x_{1}=\cdots=x_{n}=0\right\}$ como sendo o ponto base de $\mathbf{H}_{\mathbf{Q}}^{n}$. O estabilizador de $o$ em relação a $S p(n, 1)$ é

$$
S p(n) \times S p(1)=\left\{\left[\begin{array}{cc}
B & 0 \\
0 & \lambda
\end{array}\right] \in S p(n, 1): B \in S p(n) \text { e } \lambda \in S p(1)\right\} .
$$

Sejam $\mathfrak{S p}(n, 1)$ e $\mathfrak{S p}(n) \times \mathfrak{S p}(1)$ as álgebras de Lie de $S p(n, 1)$ e $S p(n) \times S p(1)$ respectivamente. Então $X \in \mathfrak{S} \mathfrak{p}(n, 1)$ é da forma

$$
X=\left[\begin{array}{ccc}
Z & \xi & \eta \\
-\xi^{*} & \nu & q \\
\eta^{*} & \bar{q} & \rho
\end{array}\right]
$$

onde $Z^{*}=-Z ; \xi, \eta \in \mathbf{Q}^{n-1} ; \nu, \rho \in \Im(\mathbf{Q})$ e $q \in \mathbf{Q}$. Por outro lado, se $X \in \mathfrak{S p}(n) \times \mathfrak{S p}(1)$ então

$$
X=\left[\begin{array}{ccc}
Z & \xi & 0 \\
-\xi^{*} & \nu & 0 \\
0 & 0 & \rho
\end{array}\right]
$$


assim obtemos a decomposição de Cartan

$$
\mathfrak{S p}(n, 1)=\mathfrak{S p}(n) \times \mathfrak{S p}(1)+\mathfrak{M}
$$

onde

$$
\mathfrak{M}=\left\{\left[\begin{array}{ccc}
0 & 0 & \eta \\
0 & 0 & q \\
\eta^{*} & \bar{q} & 0
\end{array}\right]: \eta \in \mathbf{Q}^{n-1}, q \in \mathbf{Q}\right\}
$$

Seja $H_{0}=\left[\begin{array}{lll}0 & 0 & 0 \\ 0 & 0 & 1 \\ 0 & 1 & 0\end{array}\right]$, então $\mathfrak{A}=\mathbf{R} H_{0} \subset \mathfrak{M}$ é um subespaço linear abeliano maximal. Temos que

$$
\left[H_{0}, X\right]=\left[\begin{array}{ccc}
0 & -\eta & -\xi \\
\eta^{*} & -2 \Im(q) & \rho-\nu \\
-\xi^{*} & \frac{\rho-\nu}{2 \Im} & 2 \Im)
\end{array}\right]
$$

para todo $X \in \mathfrak{S p}(n, 1)$. Portanto obtemos

(i) $\left[H_{0}, X\right]=0$ se e somente se $X=\left[\begin{array}{lll}Z & 0 & 0 \\ 0 & \nu & t \\ 0 & t & \nu\end{array}\right]$, com $t \in \mathbf{R}$ e $\nu \in \Im(\mathbf{Q})$.

(ii) $\left[H_{0}, X\right]=X$ se e somente se $X=\left[\begin{array}{ccc}0 & \xi & -\xi \\ -\xi^{*} & 0 & 0 \\ -\xi^{*} & 0 & 0\end{array}\right]$.

(iii) $\left[H_{0}, X\right]=2 X$ se e somente se $X=\left[\begin{array}{ccc}0 & 0 & 0 \\ 0 & -\rho & \rho \\ 0 & -\rho & \rho\end{array}\right]$, com $\rho \in \Im(\mathbf{Q})$.

Logo, fazendo $\mathfrak{N}=\left\{\left[\begin{array}{ccc}0 & \xi & -\xi \\ -\xi^{*} & -\rho & \rho \\ -\xi^{*} & -\rho & \rho\end{array}\right]: \xi \in \mathbf{Q}^{n-1}\right.$ e $\left.\rho \in \Im(\mathbf{Q})\right\}$ obtemos a decomposição de Iwasawa

$$
\mathfrak{S p}(n, 1)=\mathfrak{S p}(n) \times \mathfrak{S p}(1)+\mathfrak{A}+\mathfrak{N}
$$

O grupo $\exp (\mathfrak{A})$ é isomorfo a $\mathbf{R}_{+}$via

$$
\left[\begin{array}{ccc}
1_{n-1} & 0 & 0 \\
0 & \cosh t & \sinh t \\
0 & \sinh t & \cosh t
\end{array}\right] \mapsto \mathrm{e}^{t}
$$

Na outra mão, o grupo nilpotente $\mathscr{H}^{4 n-1}=\exp (\mathfrak{N})$ é chamado grupo de Heisenberg gene- 
ralizado e é parametrizado por

$$
\mathscr{H}^{4 n-1}=\left\{\left[\begin{array}{ccc}
1_{n-1} & \xi & -\xi \\
-\xi^{*} & 1-\frac{1}{2}\left(|\xi|^{2}+\nu\right) & \frac{1}{2}\left(|\xi|^{2}+\nu\right) \\
-\xi^{*} & -\frac{1}{2}\left(|\xi|^{2}+\nu\right) & 1+\frac{1}{2}\left(|\xi|^{2}+\nu\right)
\end{array}\right]: \xi \in \mathbf{Q}^{n-1} \text { e } \nu \in \Im(\mathbf{Q})\right\} .
$$

Desta maneira temos a decomposição de Iwasawa

$$
S p(n, 1)=\mathscr{H}^{4 n-1} \cdot \mathbf{R}_{+} \cdot(S p(n) \cdot S p(1))
$$

Observação 2.12. A multiplicação de matrizes em $\mathscr{H}^{4 n-1}$ induz uma estrutura de grupo no conjunto $\mathbf{Q}^{n-1} \times \Im(\mathbf{Q})$, dada por

$$
(\xi, \nu) \cdot(\eta, \rho)=\left(\xi+\eta, \nu+\rho+2 \Im\left(\xi^{*} \eta\right)\right)
$$

para todo par de elementos $(\xi, \nu),(\eta, \rho) \in \mathbf{Q}^{n-1} \times \Im(\mathbf{Q})$. Assim obtemos o isomorfismo de $\operatorname{grupos} \mathbf{Q}^{n-1} \times \Im(\mathbf{Q}) \rightarrow \mathscr{H}^{4 n-1}$

$$
(\xi, \nu) \mapsto h(\xi, \nu)=\left[\begin{array}{ccc}
1_{n-1} & \xi & -\xi \\
-\xi^{*} & 1-\frac{1}{2}\left(|\xi|^{2}+\nu\right) & \frac{1}{2}\left(|\xi|^{2}+\nu\right) \\
-\xi^{*} & -\frac{1}{2}\left(|\xi|^{2}+\nu\right) & 1+\frac{1}{2}\left(|\xi|^{2}+\nu\right)
\end{array}\right]
$$

Os elementos do grupo de Heisenberg generalizado, vistos como isometrias de $\mathbf{H}_{\mathbf{Q}}^{n}$, são chamados translações de Heisenberg.

\subsubsection{Modelos de $\mathrm{H}_{\mathrm{Q}}^{n}$}

Existem vários modelos para o espaço hiperbólico quaterniônico. Nesta seção estudamos os três mais usados na literatura. Os modelos do disco e do parabolóide são construídos intersectando $V_{-}$com hiperplanos quaterniônicos afins, a saber, um ortogonal ao eixo $x_{n+1}$, e outro paralelo a uma geratriz do cone $V_{0}$, respectivamente (vide Figura 2.3). O terceiro é construído a partir de certa folheação dada por hipersuperfícies reais chamadas horosferas (vide Figura 2.4). Em todos os casos temos coordenadas globais. Existe um quarto modelo que é bastante usado no caso real mas não assim nos demais espaços hiperbólicos, a saber o modelo do hiperbolóide. Este é na verdade o fibrado

$$
\mathbf{S}^{3} \hookrightarrow\left\{x \in \mathbf{Q}^{n+1}:\langle x, x\rangle=-1\right\} \rightarrow \mathbf{P}\left(V_{-}\right)
$$

Seja $\mathbf{D}^{n}=\left\{u \in \mathbf{Q}^{n}:(u, u)<1\right\}$ o disco aberto de dimensão real $4 n$. Consideremos o 
difeomorfismo

$$
\begin{aligned}
\mathbf{H}_{\mathbf{Q}}^{n} & \rightarrow \mathbf{D}^{n} \\
{[x] } & \mapsto u,
\end{aligned}
$$

onde

$$
u=\left[\begin{array}{c}
u_{1} \\
\vdots \\
u_{n}
\end{array}\right]=\left[\begin{array}{c}
x_{1} x_{n+1}^{-1} \\
\vdots \\
x_{n} x_{n+1}^{-1}
\end{array}\right]
$$

Dizemos que $\mathbf{D}^{n}$ é o modelo do disco unitário para $\mathbf{H}_{\mathbf{Q}}^{n}$ e as coordenadas dadas por (2.14) são chamadas coordenadas afins. Nessas coordenadas temos que a métrica e a distância são dadas por

$$
d s^{2}=\frac{\left(1-|u|^{2}\right)|d u|^{2}+|(d u, u)|^{2}}{\left(1-|u|^{2}\right)^{2}}
$$

e

$$
d(u, v)=\operatorname{arccosh} \frac{|1-(u, v)|}{\sqrt{\left[1-|u|^{2}\right]\left[1-|v|^{2}\right]}} .
$$

Note que (2.14) leva $\partial \mathbf{H}_{\mathbf{Q}}^{n}$ em $\partial \mathbf{D}^{n}=\left\{u \in \mathbf{Q}^{n}:(u, u)=1\right\} \approx \mathbf{S}^{4 n-1}$ e o ponto base $o$ na origem $0 \in \mathbf{D}^{n}$.

Consideremos os vetores $x_{\infty}=\left[\begin{array}{c}0 \\ \vdots \\ 1 \\ 1\end{array}\right], x_{0}=\left[\begin{array}{c}0 \\ \vdots \\ -1 \\ 1\end{array}\right] \in V_{0}$. Então a transformação $(2.14)$ leva $\left[x_{\infty}\right]$ e $\left[x_{0}\right]$ em $u_{\infty}=\left[\begin{array}{c}0 \\ \vdots \\ 1\end{array}\right]$ e $u_{0}=\left[\begin{array}{c}0 \\ \vdots \\ -1\end{array}\right]$ respectivamente. Seja

$$
E_{\infty}=\left\{x \in \mathbf{Q}^{n+1}:\left\langle x, x_{\infty}\right\rangle=0\right\}=\left\{x \in \mathbf{Q}^{n+1}: x_{n+1}=x_{n}\right\} \text {. }
$$

A intersecção $E \cap V_{-}$do espaço afim $E=\frac{1}{2} x_{0}+E_{\infty}$ com $V_{-}$é chamado o modelo do parabolóide de $\mathbf{H}_{\mathbf{Q}}^{n}$, e sua fronteira ideal é dada por $E \cap V_{0}$. Existem coordenadas naturais para este modelo. Com efeito, consideremos a projeção $\mathbf{Q}^{n} \rightarrow \mathbf{Q}^{n-1}$ dada por

$$
\zeta=\left[\begin{array}{c}
\zeta_{1} \\
\vdots \\
\zeta_{n-1} \\
\zeta_{n}
\end{array}\right] \mapsto \zeta^{\prime}=\left[\begin{array}{c}
\zeta_{1} \\
\vdots \\
\zeta_{n-1}
\end{array}\right]
$$

Então a transformação $\zeta \mapsto x=\left[\begin{array}{c}\zeta^{\prime} \\ \zeta_{n}-\frac{1}{2} \\ \zeta_{n}+\frac{1}{2}\end{array}\right]$, parametriza o espaço afim $E$. Ainda mais, 
tem-se

$$
\langle x, x\rangle=\left(\zeta^{\prime}, \zeta^{\prime}\right)-2 \Re\left(\zeta_{n}\right) .
$$

O conjunto $\mathscr{S}^{n}=\left\{\zeta \in \mathbf{Q}^{n}:\left(\zeta^{\prime}, \zeta^{\prime}\right)-2 \Re\left(\zeta_{n}\right)<0\right\}$ é chamado modelo de Siegel para $\mathbf{H}_{\mathbf{Q}}^{n}$. O difeomorfismo $\mathscr{S}^{n} \rightarrow \mathbf{D}^{n}$ definido por

$$
\begin{aligned}
u^{\prime} & =\zeta^{\prime}\left(\zeta_{n}+\frac{1}{2}\right)^{-1} \\
u_{n} & =\left(\zeta_{n}-\frac{1}{2}\right)\left(\zeta_{n}+\frac{1}{2}\right)^{-1},
\end{aligned}
$$

é chamado transformação de Cayley e a sua inversa é dada por

$$
\begin{aligned}
\zeta^{\prime} & =u^{\prime}\left(1-u_{n}\right)^{-1} \\
\zeta_{n} & =\frac{1}{2}\left(1+u_{n}\right)\left(1-u_{n}\right)^{-1} .
\end{aligned}
$$

A transformação de Cayley leva o conjunto $\left\{\zeta \in \mathbf{Q}^{n}:\left(\zeta^{\prime}, \zeta^{\prime}\right)-2 \Re\left(\zeta_{n}\right)=0\right\}$ em $\partial \mathbf{D}^{n}-\left\{u_{\infty}\right\}$ e aplica a origem 0 em $u_{0} \in \partial \mathbf{D}^{n}$. Definimos $\zeta_{\infty}$ como sendo o ponto ideal tal que $\partial \mathscr{S}^{n}=$ : $\left\{\zeta \in \mathbf{Q}^{n}:\left(\zeta^{\prime}, \zeta^{\prime}\right)-2 \Re\left(v_{n}\right)=0\right\} \cup\left\{\zeta_{\infty}\right\} \simeq \partial \mathbf{H}_{\mathbf{Q}}^{n}$. Note que o ponto base no modelo de Siegel é representado por $\left[\begin{array}{l}0^{\prime} \\ \frac{1}{2}\end{array}\right]$

Consideremos a forma bilinear hermitiana indefinida $\langle\langle\cdot, \cdot\rangle\rangle$ dada por

$$
\langle\langle\zeta, \eta\rangle\rangle=\left(\zeta^{\prime}, \eta^{\prime}\right)-\left[\zeta_{n}+\overline{\eta_{n}}\right]
$$

para todo par de vetores $\zeta, \eta \in \mathbf{Q}^{n}$. Então $\mathscr{S}^{n}=\left\{\zeta \in \mathbf{Q}^{n}:\langle\langle\zeta, \zeta\rangle\rangle<0\right\}$ e $\partial \mathscr{S}^{n}=\{\zeta \in$ $\left.\mathrm{Q}^{n}:\langle\langle\zeta, \zeta\rangle\rangle=0\right\} \cup\left\{\zeta_{\infty}\right\}$. Em particular, compondo a aplicação de Cayley (2.17) com a inversa de (2.14) e substituindo em (2.11) obtemos

$$
d(\zeta, \eta)=\operatorname{arccosh} \frac{|\langle\langle\zeta, \eta\rangle\rangle|}{\sqrt{\langle\langle\zeta, \zeta\rangle\rangle\langle\langle\eta, \eta\rangle\rangle}}
$$

para todo $\operatorname{par} \zeta, \eta \in \mathscr{S}$.

Seja $\gamma$ uma geodésica em $\mathbf{H}_{\mathbf{Q}}^{n}$ parametrizada pelo comprimento de arco s. Dado $p \in \mathbf{H}_{\mathbf{Q}}^{n}$, da desigualdade triangular segue que a função

$$
s \mapsto d(p, \gamma(s))-s
$$

é limitada inferiormente e estritamente decrescente. Logo a função

$$
\begin{aligned}
h_{\gamma}: \mathbf{H}_{\mathbf{Q}}^{n} & \longrightarrow \mathbf{R} \\
p & \mapsto \lim _{s \rightarrow+\infty} d(p, \gamma(s))-s,
\end{aligned}
$$

está bem definida. Dizemos que $h_{\gamma}$ é a função de Busemann para $\gamma$. Usando a função de 


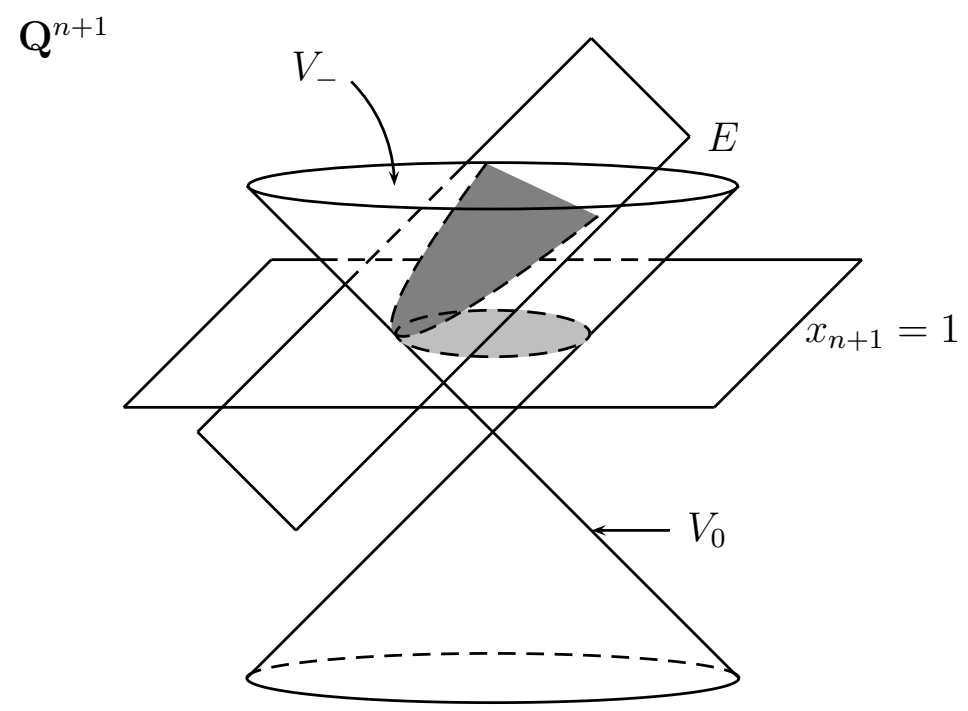

Figura 2.3: Modelos do disco e do parabóloide para $\mathbf{H}_{\mathbf{Q}}^{n}$

Busemann $h_{\gamma}$ podemos construir um outro modelo para o espaço hiperbólico quaterniônico. De fato, os conjuntos de nível de $h_{\gamma}$ constituem uma folheação $\left(H_{\alpha}\right)_{\alpha>0}$ de $\mathbf{H}_{\mathbf{Q}}^{n}$ por hipersuperfícies. Cada folha $H_{\alpha}$ é chamada horosfera. As horosferas podem ser consideradas como esferas métricas centradas no ponto $\lim _{s \rightarrow+\infty} \gamma(s) \in \partial \mathbf{H}_{\mathbf{Q}}^{n}$.

Consideremos a geodésica $\gamma$ que tem por extremos $\left[x_{0}\right],\left[x_{\infty}\right] \in \partial \mathbf{H}_{\mathbf{Q}}^{n}$, i.e.

$$
\lim _{s \rightarrow-\infty} \gamma(s)=\left[x_{0}\right] \text { e } \lim _{s \rightarrow+\infty} \gamma(s)=\left[x_{\infty}\right]
$$

No modelo de Siegel $\gamma(s)=\left[\begin{array}{c}0^{\prime} \\ \frac{1}{2} \mathrm{e}^{2 s}\end{array}\right]$. Usando (2.19), de um computo simples segue que

$$
h_{\gamma}(\zeta)=-\frac{1}{2} \ln (-\langle\langle\zeta, \zeta\rangle\rangle)
$$

Então para cada $\alpha>0$ temos que

$$
\begin{aligned}
H_{\alpha} & =h_{\gamma}^{-1}\left(-\frac{1}{2} \ln \alpha\right) \\
& =\left\{\zeta \in \mathscr{S}^{n}: h_{\gamma}(\zeta)=-\frac{1}{2} \ln \alpha\right\} \\
& =\left\{\zeta \in \mathscr{S}^{n}:\langle\langle\zeta, \zeta\rangle\rangle=-\alpha\right\} .
\end{aligned}
$$

Note que se consideramos $\alpha=0$, obtemos $H_{0}=\partial \mathscr{S}-\left\{\zeta_{\infty}\right\}$. Logo, cada horosfera é difeomorfa a $\partial \mathbf{H}_{\mathbf{Q}}^{n}-\left\{\left[x_{\infty}\right]\right\}$ (vide Observação 2.13). Ainda mais, consideremos a aplicação

$$
\begin{aligned}
\mathscr{H}^{4 n-1} & \rightarrow V_{0}-\left\{x_{\infty}\right\} \\
h(\xi, \nu) & \mapsto h(\xi, \nu)\left(\frac{1}{2} x_{0}\right) .
\end{aligned}
$$



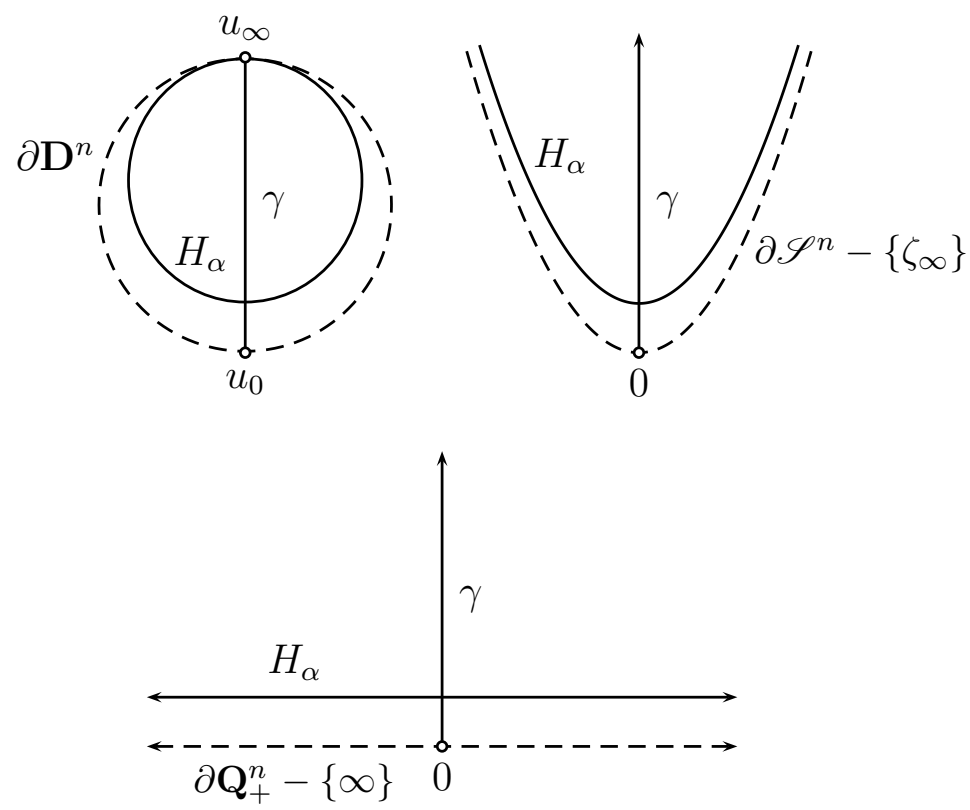

Figura 2.4: Horosferas nos diferentes modelos de $\mathbf{H}_{\mathbf{Q}}^{n}$

Esta induz uma ação livre e transitiva de $\mathscr{H}^{4 n-1}$ sobre $\partial \mathbf{H}_{\mathbf{Q}}^{n}-\left\{\left[x_{\infty}\right]\right\}$, portanto um difeomorfismo.

Assim as equações

$$
\begin{aligned}
& \omega=\zeta^{\prime}, \\
& \alpha=-\langle\langle\zeta, \zeta\rangle\rangle=2 \Re\left(\zeta_{n}\right)-\left|\zeta^{\prime}\right|^{2}, \\
& \beta=2 \Im\left(\zeta_{n}\right),
\end{aligned}
$$

definem um difeomorfismo de $\mathscr{S}^{n}$ sobre

$$
\mathbf{Q}_{+}^{n}=: \mathbf{Q}^{n-1} \times \mathbf{R}_{+} \times \Im(\mathbf{Q})
$$

Este leva a origem na origem. A imagem do ponto $\zeta_{\infty}$ é denotada por $\infty$. Observe também que esta transformação leva $\partial \mathscr{S}^{n}$ no conjunto

$$
\partial \mathbf{Q}_{+}^{n}=:\left\{(\omega, \alpha, \beta) \in \mathbf{Q}^{n-1} \times \mathbf{R} \times \Im(\mathbf{Q}): \alpha=0\right\} \cup\{\infty\}
$$

As coordenadas $(\omega, \alpha, \beta) \in \mathbf{Q}_{+}^{n}$ são chamadas coordenadas horosféricas. Nessas coordenadas

$$
H_{\alpha}=\mathbf{Q}^{n-1} \times\{\alpha\} \times \Im(\mathbf{Q})
$$

Note que o ponto base é representado por $(0,1,0)$. Finalmente, a transformação 


$$
\begin{gathered}
\mathrm{Q}_{+}^{n} \rightarrow E \cap V_{-} \\
(\omega, \alpha, \beta) \mapsto\left[\begin{array}{c}
\omega \\
q-\frac{1}{2} \\
q+\frac{1}{2}
\end{array}\right],
\end{gathered}
$$

onde $q=\frac{1}{2}(\alpha+(\omega, \omega)+\beta)$, induz um difeomorfismo de $\mathbf{Q}_{+}^{n}$ sobre $\mathbf{D}^{n}$. Assim a métrica $d s^{2}$ em coordenadas horosféricas é dada por

$$
d s^{2}=\frac{1}{4 \alpha^{2}}\left\{d \alpha^{2}-(d \beta-2 \Im((d \omega, \omega)))^{2}+4 \alpha(d \omega, d \omega)\right\}
$$

Observação 2.13. Do ponto de vista geométrico, no modelo do disco $\mathbf{D}^{n}, S p(n) \times S p(1)$ é o estabilizador da origem $0 \in \mathbf{D}^{n}$. Seja $\gamma$ a geodésica $\gamma(s)=\left[\begin{array}{c}0 \\ \vdots \\ 0 \\ \tanh s\end{array}\right] \in \mathbf{D}^{n}$. Seu centralizador é

$$
S p(n-1) \times S p(1)=\left\{\left[\begin{array}{ccc}
B & 0 & 0 \\
0 & \lambda & 0 \\
0 & 0 & \lambda
\end{array}\right] \in S p(n, 1): B \in S p(n-1) \text { e } \lambda \in S p(1)\right\} \text {. }
$$

O grupo $\mathbf{R}_{+}$é um grupo a um parâmetro de transvecções ao longo de $\gamma$, a saber cada $r \in \mathbf{R}_{+}$induz uma translação paralela $\hat{r}$ ao longo de $\gamma$, onde $\hat{r}(\gamma(s))=\gamma(s+\ln r)$. O grupo de Heisenberg generalizado $\mathscr{H}^{4 n-1}$ tem dimensão (real) $4 n-1$ e, como vimos acima, age livre e transitivamente sobre $\partial \mathbf{D}^{n}-\left\{u_{\infty}\right\}$, fixando $u_{\infty}$.

(i) Em coordenadas horosféricas temos que

(a) $h(\xi, \nu)(\omega, \alpha, \beta)=\left(\xi+\omega, \alpha, \nu+\beta+2 \Im\left(\xi^{*} \omega\right)\right)$, para $h(\xi, \nu) \in \mathscr{H}^{4 n-1}$

(b) $\hat{r}(\omega, \alpha, \beta)=\left(r \omega, r^{2} \alpha, r^{2} \beta\right)$, para $r \in \mathbf{R}_{+}$

(c) $(B, \lambda)(\omega, \alpha, \beta)=\left(B \omega \lambda^{-1}, \alpha, \lambda \beta \lambda^{-1}\right)$, se $(B, \lambda) \in S p(n-1) \times S p(1)$.

Portanto, $\mathbf{R}_{+}$age sobre $\mathbf{H}_{\mathbf{Q}}^{n}$ por dilatações levando horosferas em horosferas, mais precisamente

$$
\hat{r}\left(H_{\alpha}\right)=H_{r^{2} \alpha}
$$

(ii) No modelo de Siegel temos que
(a) $h(\xi, \nu)(\zeta)=\left[\begin{array}{c}\zeta^{\prime}+\xi \\ \zeta_{n}+\xi^{*} \zeta^{\prime}+\frac{1}{2}\left(|\xi|^{2}+\nu\right)\end{array}\right]$, para $h(\xi, \nu) \in \mathscr{H}^{4 n-1}$
(b) $\hat{r}(\zeta)=\left[\begin{array}{c}r \zeta^{\prime} \\ r^{2} \zeta_{n}\end{array}\right]$, para $r \in \mathbf{R}_{+}$
(c) $(B, \lambda)(\zeta)=\left[\begin{array}{l}B \zeta^{\prime} \lambda^{-1} \\ \lambda \zeta_{n} \lambda^{-1}\end{array}\right]$, se $(B, \lambda) \in S p(n-1) \times S p(1)$. 
(iii) No modelo do disco tem-se

(a)

$$
h(\xi, \nu)(u)=\left[\begin{array}{c}
\left(u^{\prime}-\xi\left(1-u_{n}\right)\right)\left\{\frac{\left(|\xi|^{2}+\nu\right)\left(1-u_{n}\right)}{2}+1-\xi^{*} u^{\prime}\right\}^{-1} \\
\left\{\frac{\left(|\xi|^{2}+\nu\right)\left(1-u_{n}\right)}{2}+u_{n}-\xi^{*} u^{\prime}\right\}\left\{\frac{\left(|\xi|^{2}+\nu\right)\left(1-u_{n}\right)}{2}+1-\xi^{*} u^{\prime}\right\}^{-1}
\end{array}\right]
$$

para $h(\xi, \nu) \in \mathscr{H}^{4 n-1}$

(b) $\hat{r}(u)=\left[\begin{array}{c}u^{\prime}\left(\sinh t u_{n}+\cosh t\right)^{-1} \\ \left(\cosh t u_{n}+\sinh t\right)\left(\sinh t u_{n}+\cosh t\right)^{-1}\end{array}\right]$, para $r=\mathrm{e}^{t} \in \mathbf{R}_{+}$

(c) $(B, \lambda)(u)=\left[\begin{array}{l}B u^{\prime} \lambda^{-1} \\ \lambda u_{n} \lambda^{-1}\end{array}\right]$, se $(B, \lambda) \in S p(n-1) \times S p(1)$.

\subsubsection{Bissetores e fans}

Ao contrário do que sucede no espaço hiperbólico real, em $\mathbf{H}_{\mathbf{K}}^{n} \operatorname{com} \mathbf{K}=\mathbf{C}, \mathbf{Q}$ e $\mathbf{O}$ (se $n=2$ ) não existem hipersuperfícies reais totalmente geodésicas. Como generalizações destas, em geometria hiperbólica complexa são estudados bissetores métricos (vide [Mos80] e [Gol06]). Nos casos quaterniônico e octoniônico também pode ser feito um estudo análogo (vide $[\mathrm{AK} 07]$ ).

Dado um Q-submódulo $E$ de $\mathbf{Q}^{n+1}$ cuja dimensão é $m+1$, temos que $\mathbf{P}\left(E \cap V_{-}\right)$é uma subvariedade totalmente geodésica de $\mathbf{H}_{\mathbf{Q}}^{n}$ com dimensão real $4 m$, chamada $\mathbf{Q}^{m}$-espaço. Em particular, um 2-plano quaterniônico em $\mathbf{Q}^{n+1}$ determina uma Q-linha chamada geodésica quaterniônica, cuja fronteira é chamada cadeia. A fronteira de um Q-hiperplano é dita hipercadeia. Dada uma hipercadeia $C$, a inversão em $C$ é o elemento involutivo $\iota_{C} \in$ $\mathbf{P}(S p(n, 1))$, que tem $C$ como o seu conjunto de pontos fixos, quando vista como uma transformação de $\partial \mathbf{H}_{\mathbf{Q}}^{n}$.

Exemplo 2.14. Seja $E=\left\{x \in \mathbf{Q}^{n+1}: x_{n}=0\right\}$, então, o correspondente Q-hiperplano $L$ e a hipercadeia $C=\partial L$ são dados, nos diferentes modelos de $\mathbf{H}_{\mathbf{Q}}^{n}$, por

$$
L:\left\{\begin{array}{l}
\left\{u \in \mathbf{D}^{n}: u_{n}=0\right\} \\
\left\{\zeta \in \mathscr{S}^{n}: \zeta_{n}=\frac{1}{2}\right\} \\
\left\{(\omega, \alpha, \beta) \in \mathbf{Q}_{+}^{n}:|\omega|^{2}+\alpha=1 \text { e } \beta=0\right\}
\end{array}\right.
$$

e

$$
C:\left\{\begin{array}{l}
\left\{u \in \mathbf{Q}^{n}:\left|u^{\prime}\right|=1 \text { e } u_{n}=0\right\} \\
\left\{\zeta \in \mathbf{Q}^{n}:\left|\zeta^{\prime}\right|=1 \text { e } \zeta_{n}=\frac{1}{2}\right\} \\
\left\{(\omega, \alpha, \beta) \in \mathbf{Q}^{n-1} \times \mathbf{R} \times \Im(\mathbf{Q}):|\omega|^{2}=1, \alpha=0 \text { e } \beta=0\right\}
\end{array}\right.
$$


respectivamente. Note que $\left[x_{0}\right],\left[x_{\infty}\right] \notin C$. Na outra mão, a matriz

$$
\left[\begin{array}{ccc}
1_{n-1} & 0 & 0 \\
0 & -1 & 0 \\
0 & 0 & 1
\end{array}\right] \in S p(n, 1)
$$

define a inversão $\iota=\iota_{C}$. Temos que

$$
\left\{\begin{array}{ll}
\iota(u)=\left[\begin{array}{c}
u^{\prime} \\
-u_{n}
\end{array}\right], & u \in \mathbf{D}^{n} \\
\iota(\zeta)=\left[\begin{array}{c}
\frac{1}{2} \zeta^{\prime} \zeta_{n}^{-1} \\
\frac{1}{4} \zeta_{n}^{-1}
\end{array}\right], & \zeta \in \mathscr{S}^{n} \\
\iota(\omega, \alpha, \beta)=\left(\omega\left(\alpha+|\omega|^{2}+\beta\right)^{-1}, \frac{\alpha}{\left.|\alpha+| \omega\right|^{2}+\left.\beta\right|^{2}}, \frac{-\beta}{\left.|\alpha+| \omega\right|^{2}+\left.\beta\right|^{2}}\right), & (\omega, \alpha, \beta) \in \mathbf{Q}_{+}^{n}
\end{array} .\right.
$$

Em particular, considerando $\iota: \partial \mathbf{Q}_{+}^{n} \rightarrow \partial \mathbf{Q}_{+}^{n}$, tem-se

$$
\iota(\omega, 0, \beta)=\left(\omega\left(|\omega|^{2}+\beta\right)^{-1}, 0, \frac{-\beta}{\left.|| \omega\right|^{2}+\left.\beta\right|^{2}}\right),
$$

para todo $(\omega, 0, \beta) \in \partial \mathbf{Q}_{+}^{n}-\{(0,0,0), \infty\}$. Note que $\iota$ intercambia $(0,0,0)$ e $\infty$.

Como sabemos, o fato de $\mathbf{H}_{\mathbf{Q}}^{n}$ ter curvatura seccional negativa implica que a função distância é estritamente convexa. Logo, dado um Q-espaço $L$, a aplicação $\Pi_{L}: \mathbf{H}_{\mathbf{Q}}^{n} \rightarrow L$, que leva $p$ no ponto $\Pi_{L}(p) \in L$ mais próximo de $p$, está bem definida e é chamada projeção ortogonal sobre $L$.

Definição 2.15. Dados dois pontos diferentes $p_{1}, p_{2} \in \mathbf{H}_{\mathbf{Q}}^{n}$, o bissetor equidistante de $p_{1}$ e $p_{2}$ é definido como sendo

$$
\mathscr{B}=\left\{p \in \mathbf{H}_{\mathbf{Q}}^{n}: d\left(p, p_{1}\right)=d\left(p, p_{2}\right)\right\}
$$

Seja $\Sigma$ a geodésica quaterniônica contendo $p_{1}, p_{2}$. Esta é chamada Q-espinha de $\mathscr{B}$ em relação ao par $p_{1}, p_{2}$. A intersecção $\sigma=\Sigma \cap \mathscr{B}$ é dita espinha do bissetor $\mathscr{B}$. A fronteira $\partial \mathscr{B}$ é chamada esfera espinhal. Finalmente, $\partial \sigma$ é dita esfera vortical.

Observação 2.16. (i) Note que se $E$ é o 2-plano quaterniônico gerado pelas retas $p_{1}$ e $p_{2}$, então $\Sigma=\mathbf{P}\left(E \cap V_{-}\right)$. Esta construção faz sentido ainda se $p_{1}, p_{2} \in \partial \mathbf{H}_{\mathbf{Q}}^{n}$.

(ii) A Q-espinha $\Sigma$ é isométrica a $\mathbf{H}_{\mathbf{Q}}^{1} \simeq \mathbf{H}_{\mathbf{R}}^{4}$, assim a espinha $\sigma$ é um bissetor em $\mathbf{H}_{\mathbf{R}}^{4}$, ou seja $\sigma \simeq \mathbf{H}_{\mathbf{R}}^{3}$. Portanto a esfera vortical $\partial \sigma$ é e uma cadeia difeomorfa à esfera $\mathbf{S}^{2}$. Também a esfera espinhal $\partial \mathscr{B}$ uma é hipercadeia difeomorfa à esfera $\mathbf{S}^{4 n-2}$. 
(iii) $\mathscr{B}$ fica completamente determinado por $\sigma$ (vide Lema 2.17). Portanto, um elemento de $\mathbf{P}(S p(n, 1))$ estabiliza $\mathscr{B}$ se e somente se estabiliza $\sigma$.

(iv) Por outro lado, dados dois bissetores $\mathscr{B}$ e $\mathscr{B}^{\prime}$, existe um elemento de $\mathbf{P}(S p(n, 1))$ que leva $\mathscr{B}$ sobre $\mathscr{B}^{\prime}$. Isto acontece pois $\mathbf{P}(S p(n, 1))$ age transitivamente sobre o conjunto de pares de pontos equidistantes de $\mathbf{H}_{\mathbf{Q}}^{n}$.

Lema 2.17 (Vide [AK07] e [Gol06]). Com a notação na definição 2.15 temos que

$$
\mathscr{B}=\Pi_{\Sigma}^{-1}(\sigma)=\bigcup_{p \in \sigma} \Pi_{\Sigma}^{-1}(p)
$$

onde $\Pi_{\Sigma}: \mathbf{H}_{\mathbf{Q}}^{n} \rightarrow \Sigma$ a projeção ortogonal sobre $\Sigma$. Em particular, todo bissetor está determinado pela sua esfera vortical. Além disso bissetores são folheados pelos Q-hiperplanos $S_{p}=\Pi_{\Sigma}^{-1}(p), p \in \sigma$, que são chamados slices do bissetor. Em particular bissetores são hipersuperficies regradas de $\mathbf{H}_{\mathbf{Q}}^{n}$.

Exemplo 2.18. Seja $\delta \in(0,1)$ e considere o ponto $p=\left[\begin{array}{c}0^{\prime} \\ \delta k \\ 1\end{array}\right] \in \mathbf{Q}^{n+1}$. Então, usando a equação (2.11), da Definição 2.15 segue que o bissetor $\mathscr{B}=\mathscr{B}([p],[-p])$, a Q-espinha, a espinha e a projeção ortogonal são dados, nos diferentes modelos de $\mathbf{H}_{\mathbf{Q}}^{n}$, por

$$
\begin{gathered}
\mathscr{B}:\left\{\begin{array}{l}
\left\{u \in \mathbf{D}^{n}: \Re\left(k u_{n}\right)=0\right\} \\
\left\{\zeta \in \mathscr{S}^{n}: \Re\left(k \zeta_{n}\right)=0\right\} \\
\left\{(\omega, \alpha, \beta) \in \mathbf{Q}_{+}^{n}: \Re(k \beta)=0\right\}
\end{array}\right. \\
\Sigma:\left\{\begin{array}{l}
\left\{u \in \mathbf{D}^{n}: u^{\prime}=0\right\} \\
\left\{\zeta \in \mathscr{S}^{n}: \zeta^{\prime}=0\right\} \\
\left\{(\omega, \alpha, \beta) \in \mathbf{Q}_{+}^{n}: \omega=0\right\}
\end{array}\right. \\
\sigma:\left\{\begin{array}{l}
\left\{u \in \mathbf{D}^{n}: u^{\prime}=0 \text { e } \Re\left(k u_{n}\right)=0\right\} \\
\left\{\zeta \in \mathscr{S}^{n}: \zeta^{\prime}=0 \text { e } \Re\left(k \zeta_{n}\right)=0\right\} \\
\left\{(\omega, \alpha, \beta) \in \mathbf{Q}_{+}^{n}: \omega=0 \text { e } \Re(k \beta)=0\right\}
\end{array}\right.
\end{gathered}
$$

e

$$
\Pi_{\Sigma}: \begin{cases}\Pi_{\Sigma}(u)=\left[\begin{array}{l}
0^{\prime} \\
u_{n}
\end{array}\right], & u \in \mathbf{D}^{n} \\
\Pi_{\Sigma}(\zeta)=\left[\begin{array}{l}
0^{\prime} \\
\zeta_{n}
\end{array}\right], & u \in \mathscr{S}^{n} \\
\Pi_{\Sigma}(\omega, \alpha, \beta)=\left(0, \alpha+|\omega|^{2}, \beta\right), & (\omega, \alpha, \beta) \in \mathbf{Q}_{+}^{n}\end{cases}
$$


Os slices de $\mathscr{B}$ são dados por

$$
S_{q}:\left\{\begin{array}{l}
\left\{u \in \mathbf{D}^{n}: u_{n}=q\right\} \\
\left\{\zeta \in \mathscr{S}^{n}: \zeta_{n}=q\right\} \\
\left\{(\omega, \alpha, \beta) \in \mathbf{Q}_{+}^{n}: \alpha+|\omega|^{2}=\Re(q) \text { e } \beta=\Im(q)\right\}
\end{array}\right.
$$

onde $q \in \mathbf{Q}$ com $\Re(k q)=0$ (e também $\Re(q)>0$ no último caso). Em particular, temos que $\partial \mathscr{B}$ é difeomorfo à esfera $\mathbf{S}^{4 n-2}$.

Seja $F=\left\{h(\xi, \nu) \in \mathscr{H}^{4 n-1}: \xi=0\right.$ e $\left.\Re(k \nu)=0\right\}$. Então $F \cdot \mathbf{R}_{+}$age livre e transitivamente sobre $\sigma$. Na outra mão o normalizador de $\sigma$ em $S p(n) \times S p(1)$ (vide Observação 2.13) é

$S p(n-1) \times T^{1}=\left\{\left[\begin{array}{ccc}B & 0 & 0 \\ 0 & \lambda & 0 \\ 0 & 0 & \lambda\end{array}\right] \in S p(n, 1): B \in S p(n-1)\right.$ e $\left.\lambda \in S p(1) \operatorname{com} \lambda k \lambda^{-1}=k\right\}$.

Assim pela Observação 2.16(iii) temos que o grupo

$$
F \cdot \mathbf{R}_{+} \cdot\left(S p(n-1) \times T^{1}\right)
$$

normaliza $\mathscr{B}$. Ainda mais este grupo coincide com a componente conexa da identidade do normalizador de $\mathscr{B}$. Em particular bissetores têm cohomogeneidade um. Assim parecenos que há um engano em [AK07] p.7, onde se afirma que bissetores no espaço hiperbólico quaterniônico têm cohomogeneidade três.

Lema 2.19. Bissetores são hipersuperfícies mínimas de $\mathbf{H}_{\mathbf{Q}}^{n}$.

Demonstração. Em primeiro lugar, note que se $N$ é uma subvariedade de uma variedade riemanniana $M$, então seu campo de curvatura média $\mathbb{H}$ tem a seguinte propriedade: se $\phi$ é uma isometria de $M \operatorname{com} \phi(N)=N$, então

$$
d \phi_{p} \mathbb{H}_{p}=\mathbb{H}_{\phi(p)},
$$

para todo $p \in N$. Dizemos que $\mathbb{H}$ é $\phi$-invariante.

Pela Observação 2.16(iv) basta mostrar o resultado para o bissetor $\mathscr{B}$ do Exemplo 2.18. Note que o slice de $\mathscr{B}$ passando pelo ponto base coincide com o Q-hiperplano $L$ do Exemplo 2.14. O resultado segue se mostrarmos que o campo de curvatura média $\mathbb{H}$ de $\mathscr{B}$ anula-se em $L$, pois $\mathscr{B}=\cup_{p \in L} F \cdot \mathbf{R}_{+}(p)$. Para isso consideremos a inversão $\iota$ (vide Exemplo 2.14). De fato o conjunto dos pontos fixos de $\iota$ é $L, \operatorname{logo} \mathbb{H}$ é $\iota$-invariante. Ainda mais a diferencial de $\iota$ é a identidade em vetores tangentes e é menos a identidade em vetores normais a $L$. 
Portanto, se $p \in L$ tem-se

$$
\begin{aligned}
\mathbb{H}_{p} & =\mathbb{H}_{\iota(p)} \\
& =d \iota_{p} \mathbb{H}_{p} \\
& =-\mathbb{H}_{p} .
\end{aligned}
$$

$\left.\operatorname{Logo} \mathbb{H}\right|_{L} \equiv 0$

Existe uma outra classe de hipersuperfícies reais que estão estreitamente relacionadas com bissetores, a saber fans. De fato, fans podem ser obtidos como limites de bissetores. Fans foram introduzidos em geometria hiperbólica complexa (vide [GP92]). A seguir faremos a construção análoga para o caso quaterniônico seguindo [GP92]. Como veremos depois, fans são hipersuperfícies mínimas, homogêneas e regradas (vide Proposição 2.22), todas congruentes entre si e com fronteira ideal difeomorfa à esfera $\mathbf{S}^{4 n-2}$.

Consideremos o feixe (vide Figura 2.5) das geodésicas quaterniônicas que contem $\left[x_{\infty}\right]$ ( $u_{\infty}$ no modelo do disco) na sua fronteira. Cada uma destas geodésicas está determinada por um outro ponto em $\partial \mathbf{H}_{\mathbf{Q}}^{n}$. Isto é, cada ponto $p \in \partial \mathbf{H}_{\mathbf{Q}}^{n}$ determina uma única geodésica quaterinônica $\Sigma_{p}$ tal que $\left[x_{\infty}\right] \in \partial \Sigma_{p}$ (vide Observação 2.16(i)). Este feixe tem estrutura de espaço afim quaterniônico de dimensão $n-1$. Com efeito, em coordenadas horosféricas, dados $\omega_{0} \in \mathbf{Q}^{n-1}$ e $\beta_{0} \in \Im(Q)$, a geodésica quaterniônica determinada pelos pontos $\left(\omega_{0}, 0, \beta_{0}\right), \infty \in$ $\partial \mathbf{Q}_{+}^{n}$ é

$$
\Sigma_{\omega_{0}}=\left\{(\omega, \alpha, \beta) \in \mathbf{Q}_{+}^{n}: \omega=\omega_{0}\right\} .
$$

Consideremos a projeção vertical

$$
\begin{aligned}
\Pi: \mathbf{Q}_{+}^{n} & \rightarrow \mathbf{Q}^{n-1} \\
(\omega, \alpha, \beta) & \mapsto \omega .
\end{aligned}
$$

Definição 2.20. Dado um hiperplano real afim $F \subset \mathrm{Q}^{n-1}$, a imagem inversa $\mathscr{F}=\Pi^{-1}(F)$ é chamada fan com vértice no ponto $\infty$.

Observação 2.21. Note que a inversão $\iota$ no Exemplo 2.14 intercambia $\left[x_{0}\right] \operatorname{com}\left[x_{\infty}\right]$. Assim usando $\iota$ junto com as translações de Heisenberg podemos definir fans com vértice num ponto qualquer de $\partial \mathbf{H}_{\mathbf{Q}}^{n}$.

Proposição 2.22. Fans são hipersuperfícies homogêneas, regradas, todas congruentes entre si e têm fronteira ideal homeomorfa à esfera $\mathbf{S}^{4 n-2}$.

Demonstração. Pela Observação 2.21, basta considerar fans com vértice em $\infty$. Usando translações de Heisenberg e elementos de $S p(n-1)$ vemos que todos os fans com vértice em 
$\infty$ são congruentes entre si. Consideremos o fan

$$
\mathscr{F}=\left\{(\omega, \alpha, \beta) \in \mathbf{Q}_{+}^{n}: \Re\left(\omega_{n-1}\right)=0\right\}
$$

Temos que, $\mathscr{F}=\bigcup_{\nu \in \Im(\mathbf{Q})} M_{\nu}$, onde $M_{\nu}=\left\{(\omega, \alpha, \beta) \in \mathbf{Q}_{+}^{n}: \omega_{n-1}=\nu\right\}$. Portanto, $\mathscr{F}$ é uma hiersuperfície (real) regrada pelos Q-hiperplanos $M_{\nu}, \nu \in \Im(\mathbf{Q})$. Consideremos o subgrupo $H=H^{\prime} \times \mathbf{R}_{+}$de $S p(n, 1)$, onde $H^{\prime}=\left\{h(\xi, \nu) \in \mathscr{H}^{4 n-1}: \Re\left(\xi_{n-1}\right)=0\right\}$. Então, $\mathscr{F}$ é a $H$-órbita do ponto base $(0,1,0) \in \mathbf{Q}_{+}^{n}$. Finalmente, $\partial \mathscr{F}=\left\{(\omega, \alpha, \beta) \in \mathbf{Q}^{n}\right.$ : $\Re\left(\omega_{n-1}\right)=0$, e $\left.\alpha=0\right\} \cup\{\infty\}$ é homeomorfa à esfera $\mathbf{S}^{4 n-2}$.

Exemplo 2.23. Vejamos a relação ente bissetores e fans. Consideremos o vetor

$$
e_{n-1}=\left[\begin{array}{c}
0 \\
\vdots \\
0 \\
1
\end{array}\right] \in \mathbf{Q}^{n-1}
$$

Dado $t \in \mathbf{R}$, a translação de Heisenberg $h\left(t e_{n-1}, 0\right) \in \mathscr{H}^{4 n-1}$ leva o bissetor $\mathscr{B}$ do Exemplo 2.18 no bissetor (vide Observação 2.16(iv))

$$
\mathscr{B}_{t}=h\left(t e_{n-1}, 0\right)(\mathscr{B})=\left\{(\omega, \alpha, \beta) \in \mathbf{Q}_{+}^{n}: \Re\left(k\left(\beta-2 t \omega_{n-1}\right)\right)=0\right\}
$$

Logo obtemos uma família a um parâmetro de bissetores $\left(\mathscr{B}_{t}\right)_{t \in \mathbf{R}}$, cujas esferas vorticais são da forma

$$
\partial \sigma_{t}=\left\{(\omega, \alpha, \beta) \in \mathbf{Q}^{n-1} \times \mathbf{R} \times \Im(\mathbf{Q}): \omega=t e_{n-1}, \alpha=0 \text { e } \Re(k \beta)=0\right\} \cup\{\infty\} .
$$

Assim no limite $t \rightarrow+\infty$ obtemos o fan

$$
\mathscr{F}=\left\{(\omega, \alpha, \beta) \in \mathbf{Q}_{+}^{n}: \Re\left(k \omega_{n-1}\right)=0\right\} .
$$

Note que as esferas vorticais $\partial \sigma_{t}$ colapsam no ponto $\infty$, quando $t \rightarrow+\infty$.

Por último, se $\iota$ é a inversão no Exemplo 2.14 temos que $\mathscr{F}^{\prime}=\iota(\mathscr{F})$ é um fan com vértice na origem $(0,0,0) \in \partial \mathbf{Q}_{+}^{n}$. Este fan é dado por

$$
\mathscr{F}^{\prime}=\left\{(\omega, \alpha, \beta): \Re\left(k \omega_{n-1}\left(\alpha+|\omega|^{2}+\beta\right)^{-1}\right)=0\right\} .
$$

Logo se

$$
\omega_{l}=\omega_{l, 0}+i \omega_{l, 1}+j \omega_{l, 2}+k \omega_{l, 3} \text { e } \beta=i \beta_{1}+j \beta_{2}+k \beta_{3},
$$

$\operatorname{com} \omega_{l, 0}, \omega_{l, 1}, \omega_{l, 2}, \omega_{l, 3}, \beta_{1}, \beta_{2}, \beta_{3} \in \mathbf{R}$, para $l=1, \ldots, n-1$, temos que $\mathscr{F}^{\prime}=f^{-1}(0) \cap \mathbf{Q}_{+}^{n}$, 


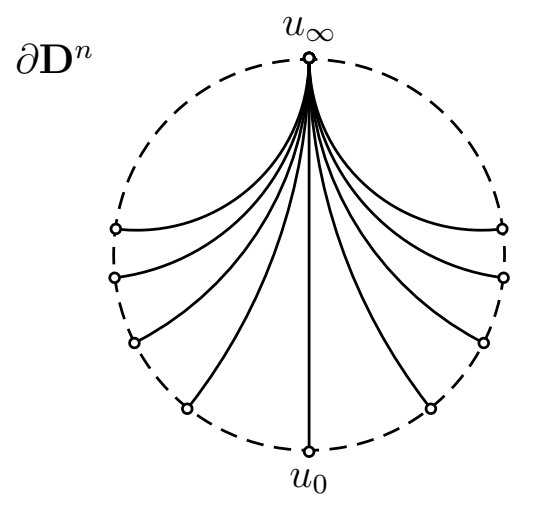

Figura 2.5: O feixe das geodésicas quaterniônicas que contêm $\left[x_{\infty}\right]$ na fronteira (no modelo do disco)

onde

$$
\begin{aligned}
f(\omega, \alpha, \beta) & =\omega_{n-1,3}\left(\alpha+\sum_{l=1}^{n-1}\left(\omega_{l, 0}^{2}+\omega_{l, 1}^{2}+\omega_{l, 2}^{2}+\omega_{l, 3}^{2}\right)\right) \\
& -\omega_{n-1,0} \beta_{3}-\omega_{n-1,1} \beta_{2}+\omega_{n-1,2} \beta_{1},
\end{aligned}
$$

para $(\omega, \alpha, \beta) \in \mathbf{Q}^{n-1} \times \mathbf{R} \times \Im(\mathbf{Q})$ (compare com [GP92], p.536). Note que o único ponto singular de $f$ em $\left\{(\omega, \alpha, \beta) \in \mathbf{Q}^{n-1} \times \mathbf{R} \times \Im(\mathbf{Q}): \alpha \geqq 0\right\}$ é $(0,0,0)$. Isto segue facilmente de calcular as derivadas parciais de $f$.

\subsubsection{Hipersuperfícies mínimas}

Finalmente, construiremos hipersuperfícies mínimas e equivariantes de $\mathbf{H}_{\mathbf{Q}}^{n}$ usando o método da geometria diferencial equivariante. Dividiremos o estudo em cinco tipos de grupos, a saber, elíptico, loxodrômico, loxodrômico especial, parabólico e parabólico especial. Em todos eles as ações são polares, logo o espaço das órbitas está mergulhado (isometricamente) em $\mathbf{H}_{\mathbf{Q}}^{n}$ (vide Capítulo §1.5). Dependendo do caso, usaremos um dos diferentes modelos de $\mathbf{H}_{\mathbf{Q}}^{n}$ para facilitar os cômputos e interpretar os resultados geometricamente.

O grupo $S p(n, 1)$ age naturalmente sobre $\partial \mathbf{D}^{n}$. Assim o Teorema de ponto fixo de Brouwer implica que todo elemento de $S p(n, 1)$ tem um ponto fixo em $\mathbf{D}^{n} \cup \partial \mathbf{D}^{n}$.

Definição 2.24. Dizemos que um elemento de $\mathbf{P}(S p(n, 1))$ é do tipo

(i) elíptico, se fixa pelo menos um ponto em $\mathbf{H}_{\mathbf{Q}}^{n}$;

(ii) loxodrômico, se fixa exatamente dois pontos em $\partial \mathbf{H}_{\mathbf{Q}}^{n}$;

(iii) parabólico, se fixa exatamente um ponto em $\partial \mathbf{H}_{\mathbf{Q}}^{n}$. 


\section{a) Caso elíptico}

Sejam $n \geq 2$ e $m=1, \ldots, n-1$. Consideremos o subgrupo $H=S p(m) \times S p(n-m)$ de $S p(n, 1)$ mergulhado diagonalmente, i.e.

$$
h=\left(h_{1}, h_{2}\right) \mapsto\left[\begin{array}{ccc}
h_{1} & 0 & 0 \\
0 & h_{2} & 0 \\
0 & 0 & 1
\end{array}\right]
$$

Se $u=\left[\begin{array}{l}\xi_{1} \\ \xi_{2}\end{array}\right] \in \mathbf{D}^{n}$ e $h \in H$, então $h(u)=\left[\begin{array}{l}h_{1} \xi_{1} \\ h_{2} \xi_{2}\end{array}\right]$. Assim, os tipos de isotropia e órbita de $\left(H, \mathbf{H}_{\mathbf{Q}}^{n}\right)$ são

$$
H_{u}= \begin{cases}S p(m) \times S p(n-m), & \text { se } u=0 \\ S p(m) \times S p(n-m-1), & \text { se } \xi_{1}=0 \\ S p(m-1) \times S p(n-m), & \text { se } \xi_{2}=0 \\ S p(m-1) \times S p(n-m-1), & \text { caso contrário }\end{cases}
$$

e

$$
H / H_{u} \approx\left\{\begin{array}{ll}
\text { um ponto, } & \text { se } u=0 \\
\mathbf{S}^{4 n-4 m-1}, & \text { se } \xi_{1}=0 \\
\mathbf{S}^{4 m-1}, & \text { se } \xi_{2}=0 \\
\mathbf{S}^{4 m-1} \times \mathbf{S}^{4 n-4 m-1}, & \text { caso contrário }
\end{array},\right.
$$

respectivamente. $\Sigma=\left\{u \in \mathbf{D}^{n}: u_{m}, u_{n} \in \mathbf{R}\right.$ e $\left.u_{l}=0, l \neq m, n\right\} \simeq \mathbf{H}_{\mathbf{R}}^{2}$ é uma seção para $\left(H, \mathbf{H}_{\mathbf{Q}}^{n}\right)$. O grupo de Weyl $W_{\Sigma}$ é $\mathbf{Z}_{2}^{2}$ e temos que o espaço das órbitas $H \backslash \mathbf{H}_{\mathbf{Q}}^{n}$ é o quadrante

$$
\Delta=\left\{(x, y) \in \mathbf{R}^{2}: x, y \geq 0 \text { e } x^{2}+y^{2}<1\right\}
$$

A projeção canônica $\pi: \mathbf{D}^{n} \rightarrow \Delta$ é dada por $\pi(u)=(x, y)$, onde $x=\left|\xi_{1}\right|$ e $y=\left|\xi_{2}\right|$. A métrica orbital e o funcional de volume no ponto $(x, y) \in \Delta$ são dados por

$$
\begin{aligned}
d s^{2} & =\frac{1}{\left(1-x^{2}-y^{2}\right)^{2}}\left\{\left(1-y^{2}\right) d x^{2}+2 x y d x d y+\left(1-x^{2}\right) d y^{2}\right\} \\
\mathscr{V}(x, y) & =\frac{x^{4 m-1} y^{4 n-4 m-1}}{\left(1-x^{2}-y^{2}\right)^{\frac{4 n+1}{2}}} .
\end{aligned}
$$

Passando a coordenadas polares $x=\tanh r \cos \theta$ e $y=\tanh r \sin \theta, \operatorname{com}(r, \theta) \in[0,+\infty] \times$ $\left[0, \frac{\pi}{2}\right]$, obtemos

$$
\begin{aligned}
d s^{2} & =d r^{2}+\sinh ^{2} r d \theta^{2} \\
\mathscr{V}(r, \theta) & =(\sinh r)^{4 n-5}(\sinh 2 r)^{3}(\sin \theta)^{4 n-8 m}(\sin 2 \theta)^{4 m-1} .
\end{aligned}
$$

Portanto do Teorema 1.18 obtemos 
Proposição 2.25. Sejam $\gamma(s)=(r(s), \theta(s))$ uma curva em $\Delta$ parametrizada pelo comprimento de arco $s$ (em relação à métrica orbital $d s^{2}$ ) e $\sigma$ o ângulo entre $\frac{\partial}{\partial r}$ e $\frac{d \gamma}{d s}$. Então a hipersuperfície $\Gamma$ de $\mathbf{H}_{\mathbf{Q}}^{n}$, com $H \backslash \Gamma=\gamma$, tem curvatura média $\mathbf{h}$ se e somente se

$$
\begin{aligned}
& \frac{d r}{d s}=\cos \sigma \\
& \frac{d \theta}{d s}=\frac{\sin \sigma}{\sinh r} \\
& \frac{d \sigma}{d s}=((4 n-8 m) \cot \theta+(8 m-2) \cot 2 \theta) \frac{\cos \sigma}{\sinh r}-((4 n-4 m) \operatorname{coth} r+6 \operatorname{coth} 2 r) \sin \sigma+\mathbf{h} .
\end{aligned}
$$

Observação 2.26. (i) Note que, no bordo $\partial \Delta=\{(x, y) \in \Delta: x=0$ ou $y=0\}$, o sistema (2.22) é singular. Porém (vide Lema 0.6), podemos considerar soluções saindo ortogonalmente de $\partial \Delta-\{(0,0)\}$.

(ii) Soluções explícitas. A curva $\theta=\arctan \sqrt{\frac{4 n-4 m-1}{4 m-1}}$ é uma solução de $(2.22)$ para $\mathbf{h} \equiv 0$. Esta gera um cone sobre $\mathbf{S}^{4 n-4 m-1} \times \mathbf{S}^{4 m-1}$, e portanto é uma hipersuperfície singular de $\mathbf{H}_{\mathbf{Q}}^{n}$. Na outra mão, se $\mathbf{h} \equiv \pm((4 n-4 m) \operatorname{coth} a+6 \operatorname{coth} 2 a)$, então a curva $r=a$ é uma solução de (2.22), que gera uma esfera métrica com centro no ponto base.

\section{b) Caso loxodrômico}

Sejam $n \geq 3$ e $m=2, \ldots, n-1$. Consideremos o subgrupo $H=S p(n-m) \times S p(m-1,1)$ de $S p(n, 1)$ mergulhado como segue

$$
h=\left(h_{1}, h_{2}\right) \mapsto\left[\begin{array}{ccc}
h_{1} & 0 & 0 \\
0 & 1 & 0 \\
0 & 0 & h_{2}
\end{array}\right] .
$$

Se $u=\left[\begin{array}{l}\xi_{1} \\ \xi_{2} \\ \xi_{3}\end{array}\right] \in \mathbf{D}^{n}, \operatorname{com} \xi_{2}=u_{n-m+1}$ e $h=\left(h_{1}, h_{2}\right) \in H$, com $h_{2}=\left[\begin{array}{ll}h_{3} & a \\ b^{*} & \rho\end{array}\right]$, onde (vide equação (2.12))

$$
\left\{\begin{array}{l}
h_{3} h_{3}^{*}-a a^{*}=1_{m-1} \\
h_{3} b-a \bar{\rho}=0 \\
b b^{*}-|\rho|^{2}=-1
\end{array} .\right.
$$


Então $h(u)=\left[\begin{array}{c}h_{1} \xi_{1}\left(b^{*} \xi_{3}+\rho\right)^{-1} \\ \xi_{2}\left(b^{*} \xi_{3}+\rho\right)^{-1} \\ \left(h_{3} \xi_{3}+a\right)\left(b^{*} \xi_{3}+\rho\right)^{-1}\end{array}\right]$. Portanto, os tipos de isotropia e órbita de $\left(H, \mathbf{H}_{\mathbf{Q}}^{n}\right)$ são

$$
H_{u}= \begin{cases}S p(n-m) \times S p(m-1) \times S p(1), & \text { se } \xi_{1}=0 \text { e } \xi_{2}=0 \\ S p(n-m) \times S p(m-1), & \text { se } \xi_{1}=0 \text { e } \xi_{2} \neq 0 \\ S p(n-m-1) \times S p(m-1) \times S p(1), & \text { se } \xi_{1} \neq 0 \text { e } \xi_{2}=0 \\ S p(n-m-1) \times S p(m-1), & \text { caso contrário }\end{cases}
$$

e

$$
H / H_{u} \approx \begin{cases}\mathbf{R}^{4 m-4} \approx \mathbf{H}_{\mathbf{Q}}^{m-4}, & \text { se } \xi_{1}=0 \text { e } \xi_{2}=0 \\ \mathbf{S}^{3} \times \mathbf{R}^{4 m-4}, & \text { se } \xi_{1}=0 \text { e } \xi_{2} \neq 0 \\ \mathbf{S}^{4 n-4 m-1} \times \mathbf{R}^{4 m-4}, & \text { se } \xi_{1} \neq 0 \text { e } \xi_{2}=0 \\ \mathbf{S}^{4 n-4 m-1} \times \mathbf{S}^{3} \times \mathbf{R}^{4 m-4}, & \text { caso contrário }\end{cases}
$$

respectivamente. $\Sigma=\left\{u \in \mathbf{D}^{n}: u_{n-m}, u_{n-m+1} \in \mathbf{R}\right.$ e $\left.u_{l}=0, l \neq n-m, n-m+1\right\} \simeq \mathbf{H}_{\mathbf{R}}^{2}$ é uma seção para $\left(H, \mathbf{H}_{\mathbf{Q}}^{n}\right)$. O grupo de Weyl $W_{\Sigma}$ é $\mathbf{Z}_{2}^{2}$ e temos que o espaço das órbitas $H \backslash \mathbf{H}_{\mathbf{Q}}^{n}$ é o quadrante

$$
\Delta=\left\{(x, y) \in \mathbf{R}^{2}: x, y \geq 0 \text { e } x^{2}+y^{2}<1\right\}
$$

A projeção canônica $\pi: \mathbf{D}^{n} \rightarrow \Delta$ é dada por $\pi(u)=(x, y)$, onde $x=\frac{\left|\xi_{2}\right|}{\sqrt{1-\left|\xi_{3}\right|^{2}}}$ e $y=\frac{\left|\xi_{1}\right|}{\sqrt{1-\left|\xi_{3}\right|^{2}}}$. A métrica orbital e o funcional de volume no ponto $(x, y) \in \Delta$ são dados por

$$
\begin{aligned}
d s^{2} & =\frac{1}{\left(1-x^{2}-y^{2}\right)^{2}}\left\{\left(1-y^{2}\right) d x^{2}+2 x y d x d y+\left(1-x^{2}\right) d y^{2}\right\}, \\
\mathscr{V}(x, y) & =\frac{x^{3} y^{4 n-4 m-1}}{\left(1-x^{2}-y^{2}\right)^{\frac{4 n+1}{2}}} .
\end{aligned}
$$

Passando a coordenadas polares $x=\tanh r \cos \theta$ e $y=\tanh r \sin \theta, \operatorname{com}(r, \theta) \in[0,+\infty] \times$ $\left[0, \frac{\pi}{2}\right]$, obtemos

$$
\begin{aligned}
d s^{2} & =d r^{2}+\sinh ^{2} r d \theta^{2}, \\
\mathscr{V}(r, \theta) & =(\sinh r)^{4 n-8 m+3}(\sinh 2 r)^{4 m-1}(\sin \theta)^{4 n-4 m-4}(\sin 2 \theta)^{3} .
\end{aligned}
$$

Portanto do Teorema 1.18 obtemos

Proposição 2.27. Sejam $\gamma(s)=(r(s), \theta(s))$ uma curva em $\Delta$ parametrizada pelo comprimento de arco s (em relação à métrica orbital $d s^{2}$ ) e $\sigma$ o ângulo entre $\frac{\partial}{\partial r}$ e $\frac{d \gamma}{d s}$. Então a 
hipersuperfície $\Gamma$ de $\mathbf{H}_{\mathbf{Q}}^{n}$, com $H \backslash \Gamma=\gamma$, tem curvatura média $\mathbf{h}$ se e somente se

$$
\begin{aligned}
\frac{d r}{d s} & =\cos \sigma \\
\frac{d \theta}{d s} & =\frac{\sin \sigma}{\sinh r} \\
\frac{d \sigma}{d s} & =((4 n-4 m-4) \cot \theta+6 \cot 2 \theta) \frac{\cos \sigma}{\sinh r} \\
& -((4 n-8 m-4) \operatorname{coth} r+(8 m-2) \operatorname{coth} 2 r) \sin \sigma+\mathbf{h} .
\end{aligned}
$$

Observação 2.28. (i) Note que, no bordo $\partial \Delta=\{(x, y) \in \Delta: x=0$ ou $y=0\}$, o sistema (2.23) é singular. Porém (vide Lema 0.6), podemos considerar soluções saindo ortogonalmente de $\partial \Delta-\{(0,0)\}$.

(ii) Soluções explícitas. A curva $\theta=\arctan \sqrt{\frac{4 n-4 m-1}{3}}$ é uma solução de $(2.23)$ para $\mathbf{h} \equiv 0$. Esta gera o produto de $\mathbf{R}^{4 m-4}$ por um cone sobre $\mathbf{S}^{4 n-4 m-1} \times \mathbf{S}^{3}$, e portanto uma hipersuperfície singular de $\mathbf{H}_{\mathbf{Q}}^{n}$. Na outra mão, se $\mathbf{h} \equiv \pm((4 n-8 m-4) \operatorname{coth} a+(8 m-$ 2 ) coth $2 a$ ), então a curva $r=a$ é uma solução de (2.23), que gera um tubo de raio constante ao redor da subvariedade totalmente geodésica $\mathbf{H}_{\mathbf{Q}}^{m-1}$.

\begin{tabular}{|c|c|c|}
\hline & Elíptico & Loxodrômico \\
\hline$A$ & $4 n-5$ & $4 n-8 m+3$ \\
\hline$B$ & 3 & $4 m-1$ \\
\hline$C$ & $4 n-8 m$ & $4 n-4 m-4$ \\
\hline$D$ & $4 m-1$ & 3 \\
\hline
\end{tabular}

Tabela 2.1: Expoentes dependendo do tipo de transformação

Demonstração do Teorema 0.2. Na verdade as equaçoẽs (2.22) e (2.23) são do mesmo tipo. Assim podemos estudá-las conjuntamente. Consideraremos o caso $\mathbf{h} \equiv 0$. Sejam $A, B, C, D$ inteiros como na Tabela 2.1. O funcional de volume é da forma

$$
\mathscr{V}(r, \theta)=(\sinh r)^{A}(\sinh 2 r)^{B}(\sin \theta)^{C}(\sin 2 \theta)^{D}
$$

Portanto, as equaçoẽs $(2.22)$ e $(2.23)$, com $\mathbf{h} \equiv 0$, são da forma

$$
\begin{aligned}
& \frac{d r}{d s}=\cos \sigma \\
& \frac{d \theta}{d s}=\frac{\sin \sigma}{\sinh r} \\
& \frac{d \sigma}{d s}=P(\theta) \frac{\cos \sigma}{\sinh r}-Q(r) \sin \sigma
\end{aligned}
$$


onde

$$
P=\frac{\partial \ln \mathscr{V}}{\partial \theta}=C \cot \theta+2 D \cot 2 \theta=(C+D) \cot \theta-D \tan \theta
$$

e

$$
Q=\frac{\partial \ln \mathscr{V}}{\partial r}+\operatorname{coth} r=(A+1) \operatorname{coth} r+2 B \operatorname{coth} 2 r=(A+B+1) \operatorname{coth} r+B \tanh r
$$

Pelas observações 2.3.5 e 2.28, podemos considerar soluções de 2.24 saindo ortogonalmente de $\partial \Delta-\{(0,0)\}$. Para cada $a>0$, seja $c_{a}(s)=\left(r_{a}(s), \theta_{a}(s), \sigma_{a}(s)\right)$ a solução de (2.24) com valores iniciais $c_{a}(0)=\left(a, 0, \frac{\pi}{2}\right)$. Consideremos a família a um parâmetro de curvas $\gamma_{a}(s)=\left(r_{a}(s), \theta_{a}(s)\right), a>0$.

Fixemos $a>0$. Multiplicando a terceira equação em (2.24) por $\sin 2 \theta$ e diferenciando em $s=0$, segue que

$$
\frac{d \sigma_{a}}{d s}(0)=-\frac{Q(a)}{2(C+D+1)}<0,
$$

pois $Q>0$. Na outra mão, da terceira equação em 2.24 , temos que

$$
\frac{d \sigma}{d s}= \begin{cases}-Q(r)<0, & \text { se } \sigma)=\frac{\pi}{2} \\ Q(r)>0, & \text { se } \sigma=-\frac{\pi}{2}\end{cases}
$$

Combinando isto com (2.25) segue que $\sigma_{a}(s) \in\left(-\frac{\pi}{2}+\delta, \frac{\pi}{2}-\delta\right)$, para algum $\delta>0$ e todo $s>0$. Em particular, da primeira equação em (2.24) segue que $r_{a}$ é uma função estritamente crescente.

Consideremos a seguinte semi-primeira integral para (2.24)

$$
I=\mathscr{V}(r, \theta) \cos \sigma
$$

Com efeito, diferenciando $I$ ao longo de uma solução de (2.24) obtemos

$$
\begin{aligned}
\frac{d I}{d s} & =\frac{\partial \mathscr{V}}{\partial r}+\mathscr{V} \operatorname{coth} r \sin ^{2} \sigma \\
& >\frac{\partial \mathscr{V}}{\partial r} \\
& >(A+2 B) \mathscr{V} \\
& \geq(A+2 B) I .
\end{aligned}
$$

Note que $A+2 B=4 n+1>0$ (vide Tabela 2.1), então segue que $\lim _{s \rightarrow+\infty} I(s)=+\infty$. Em particular $\lim _{s \rightarrow+\infty} r_{a}(s)=+\infty$ e $\theta_{a}(s) \in\left(0, \frac{\pi}{2}\right)$, para $s>0$.

Assim para cada $a>0$ construímos uma curva $\gamma_{a}$ completa e sem auto-intersecções (vide Figura 2.6) que, portanto, gera uma hipersuperície mínima, completa e mergulhada $\Gamma_{a}$ de $\mathbf{H}_{\mathbf{Q}}^{n}$. Finalmente, note que pode ser feita uma construção análoga considerando curvas saindo de $(0, \tanh a)$, com ângulo $-\frac{\pi}{2}$. 


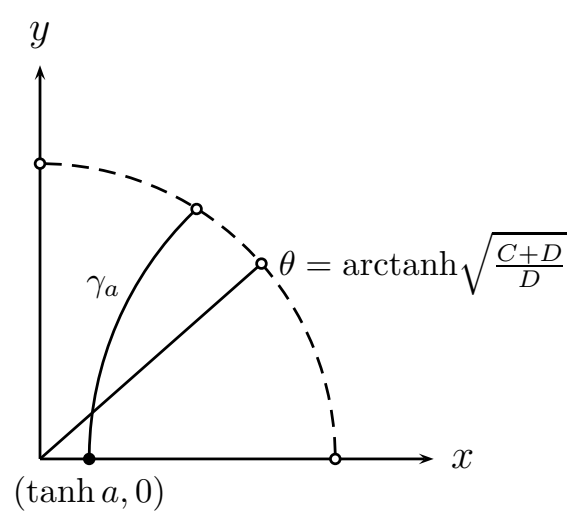

Figura 2.6: Soluções de (2.24) saindo ortogonalmente de $y=0$

\section{b') Caso loxodrômico especial}

Consideremos o subgrupo $H=F \cdot \mathbf{R}_{+} \cdot S p(n-1)$ do estabilizador do bissetor no Exemplo 2.18 , onde $S p(n-1)$ está mergulhado diagonalmente em $S p(n-1) \times S p(1)$ (vide Observação 2.13) assim

$$
B \mapsto\left[\begin{array}{ccc}
B & 0 & 0 \\
0 & 1 & 0 \\
0 & 0 & 1
\end{array}\right]
$$

Lembremos que $F=\left\{h(\xi, \nu) \in \mathscr{H}^{4 n-1}: \xi=0\right.$ e $\left.\Re(k \nu)=0\right\}$.

Observação 2.29. No caso de $\mathbf{H}_{\mathbf{C}}^{n}$, o grupo $H$ correspondente coincide com o normalizador do respectivo bissetor $\mathscr{B}=\left\{u \in \mathbf{D}^{n}: \Re\left(i u_{n}\right)=0\right\}=\left\{u \in \mathbf{D}^{n}: \Im\left(u_{n}\right)=0\right\}$, a saber $H=\mathbf{R}_{+} \times U(n-1)$. Vide [GG00].

Sejam $h(0, \nu) \in F, r=\mathrm{e}^{t} \in \mathbf{R}_{+}$e $B \in S p(n-1)$. Então se $u=\left[\begin{array}{c}u^{\prime} \\ u_{n}\end{array}\right] \in \mathbf{D}^{n}$, da Observação 2.13(iii) temos que

$$
(h(0, \nu) \cdot \hat{r} \cdot B)(u)=\left[\begin{array}{c}
B u^{\prime}\left\{\frac{\nu \mathrm{e}^{t}\left(1-u_{n}\right)}{2}+\sinh t u_{n}+\cosh t\right\}^{-1} \\
\left\{\frac{\nu \mathrm{e}^{t}\left(1-u_{n}\right)}{2}+\cosh t u_{n}+\sinh t\right\}\left\{\frac{\nu \mathrm{e}^{t}\left(1-u_{n}\right)}{2}+\sinh t u_{n}+\cosh t\right\}^{-1}
\end{array}\right]
$$

Portanto os tipos de isotropia e órbita de $\left(H, \mathbf{H}_{\mathbf{Q}}^{n}\right)$ são

$$
H_{u}= \begin{cases}\{(0,0)\} \times\{1\} \times S p(n-1), & \text { se } u^{\prime}=0 \\ \{(0,0)\} \times\{1\} \times S p(n-2), & \text { se } u^{\prime} \neq 0\end{cases}
$$

e

$$
H / H_{u} \approx\left\{\begin{array}{ll}
\mathbf{R}^{3}, & \text { se } u^{\prime}=0 \\
\mathbf{S}^{4 n-5} \times \mathbf{R}^{3}, & \text { se } u^{\prime} \neq 0
\end{array},\right.
$$

respectivamente. $\Sigma=\left\{u \in \mathbf{D}^{n}: u_{1}=\cdots u_{n-2}=0, u_{n-1}, u_{n} \in k \mathbf{R}\right\} \simeq \mathbf{H}_{\mathbf{R}}^{2}$ é uma seção para 
$\left(H, \mathbf{H}_{\mathbf{Q}}^{n}\right)$. O grupo de Weyl $W_{\Sigma}$ é $\mathbf{Z}_{2}$ e temos que o espaço das órbitas $H \backslash \mathbf{H}_{\mathbf{Q}}^{n}$ é o quadrante

$$
\Delta=\left\{(x, y) \in \mathbf{R}^{2}: x^{2}+y^{2}<1 \text { e } y \geqq 0\right\}
$$

A projeção canônica $\pi: \mathbf{D}^{n} \rightarrow \Delta$ é dada por $\pi(u)=(x, y)$, onde

$$
x=\frac{2 u_{n, 3}}{\left(1-\frac{u_{n, 1}^{2}+u_{n, 2}^{2}}{\left|1-u_{n}\right|^{2}}\right)\left(\left|1-u_{n}^{2}\right|+1-\left|u_{n}\right|^{2}\right)}
$$

$\mathrm{e}$

$$
y=\frac{2\left|u^{\prime}\right|}{\sqrt{\left(1-\frac{u_{n, 1}^{2}+u_{n, 2}^{2}}{\left|1-u_{n}\right|^{2}}\right)\left(\left|1-u_{n}^{2}\right|+1-\left|u_{n}\right|^{2}\right)}},
$$

$\operatorname{com} u_{n}=u_{n, 0}+i u_{n, 1}+j u_{n, 2}+k u_{n, 3}, u_{n, 0}, u_{n, 1}, u_{n, 2}, u_{n, 3} \in \mathbf{R}$. A métrica orbital e o funcional de volume no ponto $(x, y) \in \Delta$ são dados por

$$
\begin{aligned}
d s^{2} & =\frac{1}{\left(1-x^{2}-y^{2}\right)^{2}}\left\{\left(1-y^{2}\right) d x^{2}+2 x y d x d y+\left(1-x^{2}\right) d y^{2}\right\} \\
\mathscr{V}(x, y) & =\frac{\left(1+x^{2}\right)^{3} y^{4 n-5}}{\left(1-x^{2}-y^{2}\right)^{\frac{4 n+1}{2}}} .
\end{aligned}
$$

Passando a coordenadas polares $x=\tanh r \cos \theta$ e $y=\tanh r \sin \theta, \operatorname{com}(r, \theta) \in[0,+\infty] \times$ $[0, \pi]$, obtemos

$$
\begin{aligned}
d s^{2} & =d r^{2}+\sinh ^{2} r d \theta^{2} \\
\mathscr{V}(r, \theta) & =\left(\cosh ^{2} r+\sinh ^{2} r \cos ^{2} \theta\right)^{3}(\sinh r)^{4 n-5}(\sin \theta)^{4 n-5} .
\end{aligned}
$$

Portanto, do Teorema 1.18 obtemos

Proposição 2.30. Sejam $\gamma(s)=(r(s), \theta(s))$ uma curva em $\Delta$ parametrizada pelo comprimento de arco $s$ (em relação à métrica orbital $d s^{2}$ ) e $\sigma$ o ângulo entre $\frac{\partial}{\partial r}$ e $\frac{d \gamma}{d s}$. Então a hipersuperfície $\Gamma$ de $\mathbf{H}_{\mathbf{Q}}^{n}$, com $H \backslash \Gamma=\gamma$, tem curvatura média $\mathbf{h}$ se e somente se

$$
\begin{aligned}
\frac{d r}{d s} & =\cos \sigma \\
\frac{d \theta}{d s} & =\frac{\sin \sigma}{\sinh r} \\
\frac{d \sigma}{d s} & =\left((4 n-5) \cot \theta-3 \frac{\sinh ^{2} r \sin 2 \theta}{\cosh ^{2} r+\sinh ^{2} r \cos ^{2} \theta}\right) \frac{\cos \sigma}{\sinh r} \\
& -\left((4 n-4) \operatorname{coth} r+3 \frac{\sinh ^{2} r\left(1+\cos ^{2} \theta\right)}{\cosh ^{2} r+\sinh ^{2} r \cos ^{2} \theta}\right) \sin \sigma+\mathbf{h} .
\end{aligned}
$$

Observação 2.31. (i) Note que, no bordo $\partial \Delta=\left\{(x, y) \in \mathbf{R}^{2}: y=0\right\}$, o sistema (2.26) é singular. Porém (vide Lema 0.6), podemos considerar soluções saindo ortogonalmente de $\partial \Delta$. 
(ii) Soluções explícitas. Note que a métrica orbital e o funcional de volume são invariantes pela reflexão na reta $\theta=\frac{\pi}{2}$ (ou equivalentemente a reta $x=0$ ). Em particular, esta reta é uma solução de (2.26), para $\mathbf{h} \equiv 0$, que gera o bissetor do Exemplo 2.18.

Demonstração do Teorema 0.3. Consideremos o sistema (2.26) com $\mathbf{h} \equiv 0$. Temos que a terceira equação em (2.26) é da forma (vide Observação 1.19(ii))

$$
\frac{d \sigma}{d s}=P(r, \theta) \frac{\cos \sigma}{\sinh r}-Q(r, \theta) \sin \sigma
$$

onde

$$
P(r, \theta)=\frac{\partial \ln \mathscr{V}}{\partial \theta}=(4 n-5) \cot \theta-3 \frac{\sinh ^{2} r \sin 2 \theta}{\cosh ^{2} r+\sinh ^{2} r \cos ^{2} \theta}
$$

$\mathrm{e}$

$$
Q(r, \theta)=\frac{\partial \ln \mathscr{V}}{\partial r}+\operatorname{coth} r=(4 n-4) \operatorname{coth} r+3 \frac{\sinh 2 r\left(1+\cos ^{2} \theta\right)}{\cosh ^{2} r+\sinh ^{2} r \cos ^{2} \theta}
$$

Pela Observação 2.31(i) podemos considerar soluções de (2.26) saindo ortogonalmente do bordo $\partial \Delta$. Para cada $a \in \mathbf{R}$ considere a solução $c_{a}(s)=\left(r_{a}(s), \theta_{a}(s), \sigma_{a}(s)\right)$ de $(2.26)$ com valores iniciais

$$
c_{a}(0)= \begin{cases}\left(a, 0, \frac{\pi}{2}\right), & \text { se } a>0 \\ \left(0,0, \frac{\pi}{2}\right), & \text { se } a=0 \\ \left(a, 0,-\frac{\pi}{2}\right), & \text { se } a<0\end{cases}
$$

Seja $\gamma_{a}=\left(r_{a}(s), \theta_{a}(s)\right)$. Então $\gamma_{0}$ corresponde à reta $\theta=\frac{\pi}{2}$ e $\gamma_{-a}$ é obtida de $\gamma_{a}$ pela reflexão em relação a $\gamma_{0}$ (vide Observação 2.31(ii)). Assim basta considerar $a>0$. Fixemos $a>0$. Procedendo como na demosnstração do Teorema 0.2 vejamos que $\gamma_{a}$ está definida para todo $s \geq 0$ sem auto-interseções (vide Figura 2.7). Primeiro, multiplicando (2.27) por $\sin \theta$ e diferenciando em $s=0$ temos que $\frac{d \sigma_{a}(s)}{d s}(0)=-\frac{Q(a, 0)}{4 n-4}<0$, pois $Q>0$. Na outra mão de (2.27) tem-se

$$
\frac{d \sigma}{d s}=\left\{\begin{array}{ll}
Q(r, \theta)>0, & \text { se } \sigma=\frac{\pi}{2} \\
-Q(r, \theta)<0, & \text { se } \sigma=-\frac{\pi}{2}
\end{array} .\right.
$$

Segue que $\sigma_{a} \in\left(-\frac{\pi}{2}+\delta, \frac{\pi}{2}-\delta\right)$, para algum $\delta>0$ e todo $s \geq 0$. Em particular, da primeira equação em (2.26), tem-se que $r_{a}$ é uma função estritamente crescente. Considerando a função $I=\mathscr{V}(r, \theta) \cos \sigma$ e computando sua derivada ao longo de uma solução de (2.26) vemos que $\lim _{s \rightarrow+\infty} r_{a}(s)=+\infty$.

Para ver que a família $\left(\Gamma_{a}\right)_{a \in \mathbf{R}}$ é analítica em $a$ usamos as coordenadas cartesianas $(x, y)$. Com efeito, perto da reta $y=0$ podemos considerar soluções de $(2.26)$ da forma $x=x(y)$, 


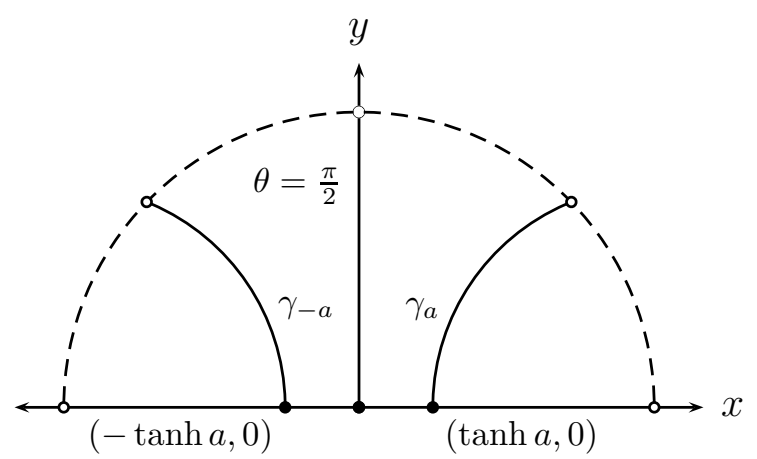

Figura 2.7: Soluções de (2.26) saindo ortogonalmente de $y=0$, com $\mathbf{h} \equiv 0(a>0)$

nesse caso (2.26) se transforma em

$$
\begin{aligned}
y \frac{d^{2} x}{d y^{2}} & =\frac{1}{\left(1+x^{2}\right)\left(1-x^{2}-y^{2}\right)}\left(\left(1-y^{2}\right)\left(\frac{d x}{d y}\right)^{2}+2 x y \frac{d x}{d y}+1-x^{2}\right) \\
& \times\left(12 x y-\left((4 n-5)\left(1+x^{2}\right)+6 y^{2}\right) \frac{d x}{d y}\right) .
\end{aligned}
$$

A Proposição 1 em [HH82] mostra que existe uma única solução analítica $x=x(t, y)$ para (2.28) a qual é uma serie de potências de $(t, y)$ numa vizinhança de $\left(t_{0}, 0\right)$ com $x\left(t_{0}, 0\right)=t_{0}$ e $\frac{d x}{d y}\left(t_{0}, 0\right)=0$, para todo $t_{0} \in(0,1)$.

Finalmente, seja $\Gamma_{a}$ a hipersuperfície gerada por $\gamma_{a}$. Da Observação 2.31(ii) temos que $\Gamma_{0}$ é o bissetor do Exemplo 2.18. Suponhamos que $\Gamma_{a}$ é um bissetor para $a \neq 0$. Temos que $H=F \cdot \mathbf{R}_{+} \cdot S p(n-1)$ estabiliza $\Gamma_{a}$ e portanto estabiliza a sua espinha (vide Observação 2.16(iii)). Assim, as espinhas de $\Gamma_{a}$ e $\Gamma_{0}$ coincidem e portanto do Lema 2.17 segue que $\Gamma_{a}=\Gamma_{0}$.

\section{c) Caso parabólico}

Sejam $n \geq 2$ e $m=1, \ldots, n-1$. Denotemos com $\mathscr{H}^{4 m-1}$ o subgrupo do grupo de Heisenberg generalizado dado por

$$
\mathscr{H}^{4 m-1}=\left\{h(\xi, \nu) \in \mathscr{H}^{4 n-1}: \xi=\left[\begin{array}{l}
0 \\
\eta
\end{array}\right], \operatorname{com} \eta \in \mathbf{Q}^{m-1}\right\} .
$$

Seja $S p(n-m)$ mergulhado diagonalmente em $S p(n, 1)$ por

$$
B \mapsto\left[\begin{array}{cc}
B & 0 \\
0 & 1_{m+1}
\end{array}\right]
$$

onde $1_{m+1}$ denota a matriz identidade de ordem $m+1$. Consideremos o subgrupo $H=$ $S p(n-m) \times \mathscr{H}^{4 m-1}$ de $S p(n, 1) . \operatorname{Dados} p=(\omega, \alpha, \beta) \in \mathbf{Q}_{+}^{n}, \operatorname{com} \omega=\left[\begin{array}{l}u \\ v\end{array}\right]$, onde $u \in \mathbf{Q}^{n-m}$ 
e $v \in \mathbf{Q}^{m-1} ;$ se $(B, h(\xi, \nu)) \in H$ temos que (vide Observação 2.13(i))

$$
(B, h(\xi, \nu))(p)=\left(\left[\begin{array}{c}
B u \\
v+\eta
\end{array}\right], \alpha, \beta+\nu+2 \Im\left(\eta^{*} v\right)\right) .
$$

Portanto os tipos de isotropia e órbita de $\left(H, \mathbf{H}_{\mathbf{Q}}^{n}\right)$ são

$$
H_{p}= \begin{cases}S p(n-m), & \text { se } u=0 \\ S p(n-m-1), & \text { se } u \neq 0\end{cases}
$$

e

$$
H / H_{p}= \begin{cases}\mathbf{R}^{4 m-1}, & \text { se } u=0, \\ \mathbf{S}^{4 n-4 m-1} \times \mathbf{R}^{4 m-1}, & \text { se } u \neq 0,\end{cases}
$$

respectivamente. $\Sigma=\left\{p \in \mathbf{Q}_{+}^{n}: u_{n-m} \in \mathbf{R}, u_{l}=0\right.$ se $l \neq n-m, v=0, \beta=0$ e $\alpha \in$ $\left.\mathbf{R}_{+}\right\} \simeq \mathbf{H}_{\mathbf{R}}^{2}$ é uma seção para $\left(H, \mathbf{H}_{\mathbf{Q}}^{n}\right)$. O grupo de Weyl $W_{\Sigma}$ é $\mathbf{Z}_{2}$ e temos que o espaço das órbitas $H \backslash \mathbf{H}_{\mathbf{Q}}^{n}$ é o quadrante

$$
\Delta=\left\{(\alpha, \rho) \in \mathbf{R}^{2}: \alpha>0 \text { e } \rho \geq 0\right\}
$$

A projeção canônica é dada por $\pi(p)=(\alpha, \rho)$, onde $\rho=|u|$. A métrica orbital e o funcional de volume no ponto $(\alpha, \rho) \in \Delta$ são dados por

$$
\begin{aligned}
d s^{2} & =\frac{1}{4 \alpha^{2}}\left(d \alpha^{2}+4 \alpha d \rho^{2}\right) \\
\mathscr{V}(\alpha, \rho) & =\alpha^{-\frac{4 n+1}{2}} \rho^{4 n-4 m-1}
\end{aligned}
$$

Portanto do Teorema 1.18 obtemos

Proposição 2.32. Sejam $\gamma(s)=(\alpha(s), \rho(s))$ uma curva em $\Delta$ parametrizada pelo comprimento de arco s (em relação à métrica orbital $d s^{2}$ ) e $\sigma$ o ângulo entre $\frac{\partial}{\partial \alpha}$ e $\frac{d \gamma}{d s}$. Então a hipersuperfície $\Gamma$ de $\mathbf{H}_{\mathbf{Q}}^{n}$, com $H \backslash \Gamma=\gamma$, tem curvatura média $\mathbf{h}$ se e somente se

$$
\begin{aligned}
& \frac{d \alpha}{d s}=2 \alpha \cos \sigma \\
& \frac{d \rho}{d s}=\sqrt{\alpha} \sin \sigma \\
& \frac{d \sigma}{d s}=(4 n-4 m-1) \frac{\sqrt{\alpha}}{\rho} \cos \sigma+(4 n+2) \sin \sigma+\mathbf{h} .
\end{aligned}
$$

Observação 2.33. (i) Note que o sistema (2.29) é singular no bordo $\partial \Delta=\{(\alpha, \rho) \in \Delta$ : $\rho=0\}$. Porém podemos considerar soluções saindo ortogonalmente de $\partial \Delta$ (vide Lema $0.6)$.

(ii) Soluções explícitas. Note que para $\mathbf{h} \equiv \pm(4 n+2)$, as curvas $\alpha$ constante, são soluções 
de (2.29). Estas geram horosferas.

(iii) Note que $\mathbf{R}_{+}$normaliza $H$. Então este grupo induz as dilatações

$$
(\alpha, \rho, \sigma) \mapsto\left(r^{2} \alpha, r \rho, \sigma\right)
$$

$r \in \mathbf{R}_{+}$, que deixam (2.29) invariante.

\section{c') Caso parabólico especial}

Seja $H=\left\{h(\xi, \nu) \in \mathscr{H}^{4 n-1}: \Re\left(\xi_{n-1}\right)=0\right\}$. Da Observação 2.13(i), segue que se $(\omega, \alpha, \beta) \in \mathbf{Q}_{+}^{n}$ e $h(\xi, \nu) \in H$

$$
h(\xi, \nu)(\omega, \alpha, \beta)=\left(\omega+\xi, \alpha, \beta+\nu+2 \Im\left(\xi^{*} \omega\right)\right) .
$$

Então, a isotropia em todo ponto é trivial e portanto o tipo de órbita de $\left(H, \mathbf{H}_{\mathbf{Q}}^{n}\right)$ é $H \approx$ $\mathbf{R}^{4 n-2} . \Sigma=\left\{(\omega, \alpha, \beta) \in \mathbf{Q}_{+}^{n}: \omega_{n-1} \in \mathbf{R}, \omega_{l}=0\right.$ se $l \neq n-1, \beta=0$ e $\left.\alpha \in \mathbf{R}_{+}\right\} \simeq \mathbf{H}_{\mathbf{R}}^{2}$ é uma seção para $\left(H, \mathbf{H}_{\mathbf{Q}}^{n}\right)$. O grupo de Weyl $W_{\Sigma}$ é trivial, assim o espaço das órbitas $H \backslash \mathbf{H}_{\mathbf{Q}}^{n}$ é o semi-plano $\Delta=\left\{(\alpha, \rho) \in \mathbf{R}^{2}: \alpha>0\right\}$. A projeção canônica é dada por $\pi(\omega, \alpha, \beta)=(\alpha, \rho)$, onde $\rho=\Re\left(\omega_{n-1}\right)$. A métrica orbital e o funcional de volume no ponto $(\alpha, \rho) \in \Delta$ são dados por

$$
\begin{aligned}
d s^{2} & =\frac{1}{4 \alpha^{2}}\left(d \alpha^{2}+4 \alpha d \rho^{2}\right) \\
\mathscr{V}(\alpha, \rho) & =\alpha^{-\frac{4 n+1}{2}}
\end{aligned}
$$

Portanto, do Teorema 1.18 obtemos

Proposição 2.34. Sejam $\gamma(s)=(\alpha(s), \rho(s))$ uma curva em $\Delta$ parametrizada pelo comprimento de arco $s$ (em relação à métrica orbital $d s^{2}$ ) e $\sigma$ o ângulo entre $\frac{\partial}{\partial \alpha}$ e $\frac{d \gamma}{d s}$. Então a hipersuperfície $\Gamma$ de $\mathbf{H}_{\mathbf{Q}}^{n}$, com $H \backslash \Gamma=\gamma$, tem curvatura média $\mathbf{h}$ se e somente se

$$
\begin{aligned}
& \frac{d \alpha}{d s}=2 \alpha \cos \sigma \\
& \frac{d \rho}{d s}=\sqrt{\alpha} \sin \sigma \\
& \frac{d \sigma}{d s}=(4 n+2) \sin \sigma+\mathbf{h} .
\end{aligned}
$$

Observação 2.35. (i) Soluções explícitas. Note que, a métrica modificada $d \bar{s}^{2}=\mathscr{V}^{2} d s^{2}$ é invariante pelas reflexões nas retas $\rho$ constante. Em particular, tais retas são soluções de (2.30), para $\mathbf{h} \equiv 0$, que geram fans com vértice no ponto no infinito (vide Definição 2.20). Na outra mão, se $\mathbf{h} \equiv \pm(4 n+2)$, as retas $\alpha$ constante são soluções de $(2.30)$ que geram horosferas. 


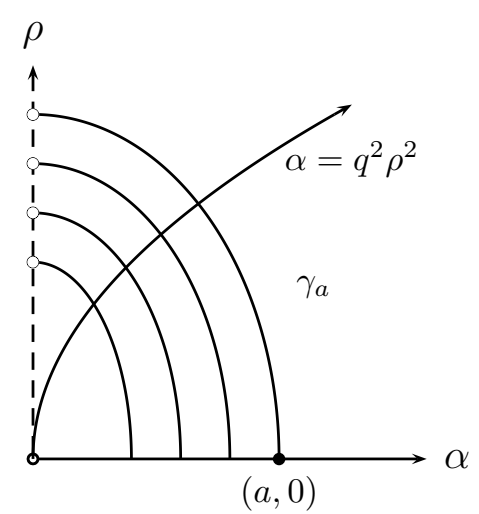

Figura 2.8: Soluções de (2.29), para $\mathbf{h} \equiv 0$, saindo ortogonalmente de $\rho=0$

(ii) Note que, $\mathbf{R}_{+}$normaliza $H$. Então este grupo induz as dilatações

$$
(\alpha, \rho, \sigma) \mapsto\left(r^{2} \alpha, r \rho, \sigma\right)
$$

$r \in \mathbf{R}_{+}$; de deixam (2.30) invariante.

(iii) Na outra mão, (2.30) também é invariante por transalações em $\rho$ (estas transformações são induzidas por $\left.\mathscr{H}^{4 n-1} / H\right)$.

Demonstração do Teorema 0.4. (i) Caso parabólico. Primeiramente consideremos o sistema (2.29) para $\mathbf{h} \equiv 0$.

(a) Pontos críticos de $\alpha, \rho$ e $\sigma$. Diferenciando a primeira equação de (2.29) temos que $\frac{d^{2} \alpha}{d s^{2}}=-(8 n+4) \alpha<0$, se $\frac{d \alpha}{d s}=0$. Então os pontos críticos (locais) de $\alpha$ são de máximo.

Diferenciando a segunda equação de $(2.29)$ temos que $\frac{d^{2} \rho}{d s^{2}}=(4 n-4 m-1) \frac{\alpha}{\rho}>0$, se $\frac{d \rho}{d s}=0$. Então os pontos críticos (locais) de $\rho$ são de mínimo.

Diferenciando a terceira equação de (2.29), para temos que

$$
\begin{aligned}
\frac{d^{2} \sigma}{d s^{2}}= & \frac{\sqrt{\alpha}}{\rho}\left((4 n-4 m-1) \cos ^{2} \sigma+(4 n+2) \sin ^{2} \sigma\right) \\
& +\frac{d \sigma}{d s}\left((4 n+2) \cos \sigma-(4 n-4 m) \frac{\sqrt{\alpha}}{\rho} \sin \sigma\right)
\end{aligned}
$$

Segue que os pontos críticos (locais) de $\sigma$ são de mínimo.

(b) Soluções de (2.29) saindo do bordo (vide Observação 2.33). Para cada $a>0$ seja $c_{a}(s)=\left(\alpha_{a}(s), \rho_{a}(s), \sigma_{a}(s)\right)$ a solução de $(2.29)$ com condição inicial $c_{a}(0)=\left(a, 0, \frac{\pi}{2}\right)$. Consideremos a família a um parâmetro de curvas $\gamma_{a}(s)=\left(\alpha_{a}(s), \rho_{a}(s)\right), a>0$ (vide Figura $2.8)$.

Multiplicando a terceira equação de (2.29) por $\rho$ e diferenciando em $s=0$ tem-se

$$
\frac{d \sigma_{a}}{d s}(0)=\frac{4 n+2}{4 n-4 m}>0
$$


Na outra mão, suponhamos que $\sigma_{a}\left(s^{\prime}\right)=\pi$, para algum $s^{\prime}>0$. Então da terceira equação em (2.29) segue que $\frac{d \sigma_{a}}{d s}\left(s^{\prime}\right)<0$. Portanto de (a) segue que $\sigma_{a}(s) \in\left(\frac{\pi}{2}, \pi\right)$, para $s>0$ e que $\sigma_{a}$ é estritamente crescente. Então o $\operatorname{limite}_{\lim _{s \rightarrow \infty}} \sigma_{a}(s)$ existe e pertence ao intervalo $\left(\frac{\pi}{2}, \pi\right]$. Em particular, da primeira equação em (2.29) temos que $\alpha_{a}$ é estritamente decrescente. Ainda mais, como e limitada inferiormente então

$$
0=\lim _{s \rightarrow+\infty} \frac{d \alpha_{a}}{d s}(s)=2 \lim _{s \rightarrow+\infty} \alpha_{a}(s) \cos \lim _{s \rightarrow+\infty} \sigma_{a}(s)
$$

Portanto,

$$
\lim _{s \rightarrow+\infty} \alpha_{a}(s)=0 .
$$

Da segunda equação em (2.29) temos que $\rho_{a}$ é estritamente crescente, logo

$$
\lim _{s \rightarrow+\infty} \frac{\sqrt{\alpha_{a}(s)}}{\rho_{a}(s)} \cos \sigma_{a}(s)=0
$$

Então, da terceira equação em $(2.29)$ segue que $0=(4 n+2) \lim _{s \rightarrow+\infty} \sin \sigma_{a}$. Assim

$$
\lim _{s \rightarrow+\infty} \sigma_{a}(s)=\pi
$$

Agora, usaremos semi-primeiras integrais de (2.29) para mostrar que $\lim _{s \rightarrow+\infty} \rho_{a}(s)$ existe, ainda mais construiremos um intervalo que contém esse limite. Com efeito, considere as funções

$$
I=\alpha^{-2 n-1} \rho^{\frac{(4 n+2)(4 n-4 m-2)+1}{4 n+1}}\left(\sqrt{\alpha} \cos \sigma+\frac{4 n+1}{4 n-4 m-1} \rho \sin \sigma\right)
$$

$\mathrm{e}$

$$
J=\alpha^{-\frac{(4 n-4 m-1)(2 n+1)}{4 n-4 m}} \rho^{4 n-4 m-1}\left(\sqrt{\alpha} \cos \sigma+\frac{4 n+2}{4 n-4 m} \rho \sin \sigma\right) .
$$

Então, diferenciando $I$ e $J$ ao longo de uma solução de 2.29 com $\rho(0)=0$, tem-se

$$
\frac{d I}{d s}=-\frac{4 m+2}{4 n+1} \alpha^{-2 n} \rho^{\frac{(4 n+2)(4 n-4 m-2)+1}{4 n+1}} \cos \sigma \sin \sigma<0
$$

$\mathrm{e}$

$$
\frac{d J}{d s}=\frac{(4 n+2)^{2}}{(4 n-4 m)^{2}} \alpha^{-\frac{(4 n-4 m-1)(2 n+1)}{4 n-4 m}} \rho^{4 n-4 m} \sin \sigma \cos \sigma>0,
$$

para $s>0$. Assim, como $I(0)=J(0)=0$, temos que $I(s)<0$ e $J(s)>0$, para $s>0$. Portanto

$$
-\frac{4 n-4 m}{4 n+2} \frac{\sqrt{\alpha_{a}}}{\rho_{a}}<\tan \sigma_{a}<-\frac{4 n-4 m-1}{4 n+1} \frac{\sqrt{\alpha_{a}}}{\rho_{a}},
$$

para todo $a>0$. Logo, das primeiras duas equações em (2.29), segue que

$$
-\frac{4 n-4 m}{4 n+2} \frac{d \alpha_{a}}{d s}<2 \rho_{a} \frac{d \rho_{a}}{d s}<-\frac{4 n-4 m-1}{4 n+1} \frac{d \alpha_{a}}{d s} .
$$




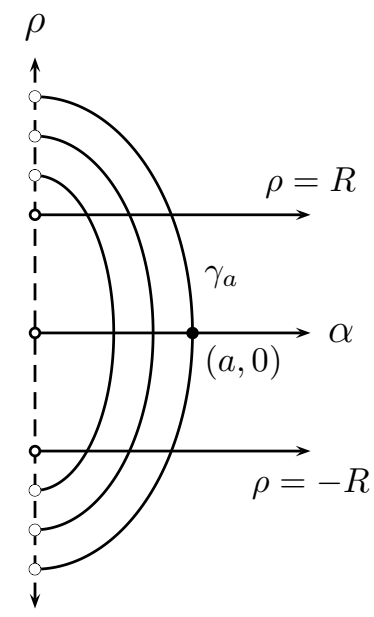

Figura 2.9: Soluções de (2.30) para $\mathbf{h} \equiv 0$

Integrando obtemos

$$
\frac{4 n-4 m}{4 n+2}\left(a-\alpha_{a}(s)\right) \leq \rho_{a}(s)^{2} \leq \frac{4 n-4 m-1}{4 n+1}\left(a-\alpha_{a}(s)\right) .
$$

Então, temos que

$$
\sqrt{\frac{4 n-4 m}{4 n+2} a} \leq \lim _{s \rightarrow+\infty} \rho_{a}(s) \leq \sqrt{\frac{4 n-4 m-1}{4 n+1} a} .
$$

(c) Folheações. Consideremos a família de arcos de parábola $\alpha=q^{2} \rho^{2}, q \in(0,+\infty]$. Temos que a curva $\gamma_{a}$ intersecta cada arco de parábola num único ponto (vide Figura 2.8). Na outra mão, da Observação 2.35(ii), temos que as dilatações $(\alpha, \rho) \mapsto\left(r^{2} \alpha, r \rho\right)$ levam $\gamma_{a}$ em $\gamma_{r^{2} a}$ deixando $\sigma_{a}$ invariante. Além disso, essas transformações fixam cada arco de parábola. Portanto, concluímos que duas curvas $\gamma_{a}$ e $\gamma_{a^{\prime}}$ não podem se intersectar se $a \neq a^{\prime}$. Assim a família $\left(\gamma_{a}\right)_{a>o}$ constitui uma folheação de $\Delta$. Segue que a família de hipersuperfícies geradas $\left(\Gamma_{a}\right)_{a>0}$ constitui uma folheação de $\mathbf{H}_{\mathbf{Q}}^{n}$, invariante pelo grupo a um parâmetro de transvecções induzido pelas translações em $\rho$ (vide Observação 2.35(iii)).

(d) Topologia de $\partial \Gamma_{a}$. Fixemos $a>0$ e sejam $\Gamma=\Gamma_{a}$ e $R=\lim _{s \rightarrow+\infty} \rho_{a}(s)$. Seja $p \in \partial \mathbf{H}_{\mathbf{Q}}^{n}$ o ponto correspondente ao ponto $(0, R) \in\left\{(\alpha, \rho) \in \mathbf{R}^{2}: \alpha=0\right.$ e $\left.\rho>0\right\}$. Então $\partial \Gamma$ é o fecho da órbita de $p$. No modelo de Siegel temos que

$$
\begin{aligned}
\partial \Gamma & =\left\{\zeta \in \partial \mathscr{S}^{n}:\left|\zeta_{1}\right|^{2}+\cdots+\left|\zeta_{n-m}\right|^{2}=R^{2}\right\} \cup\left\{\zeta_{\infty}\right\} \\
& =\left\{\zeta \in \mathbf{Q}^{n}:\left|\zeta_{1}\right|^{2}+\cdots+\left|\zeta_{n-m}\right|^{2}=R^{2}=2 \Re\left(\zeta_{n}\right)-\left|\zeta_{n-m+1}\right|^{2}-\cdots-\left|\zeta_{n-1}\right|^{2}\right\} \cup\left\{\zeta_{\infty}\right\}
\end{aligned}
$$

Isto mostra que $\partial \Gamma$ é uma variedade pinçada de Hopf do tipo $(4 m-1,4 n-4 m-1)$. Assim concluímos a prova do item (i) do Teorema 0.4 . 
(ii) Caso parabólico especial. Agora, estudemos a equação (2.30), para $\mathbf{h} \equiv 0$. Consideremos a função $I=\alpha^{-2 n-1} \sin \sigma$. Diferenciando ao longo de uma solução de (2.30) temos que

$$
\frac{d I}{d s}=0
$$

Portanto $I$ é uma primeira integral para (2.30), i.e. I é constante ao longo de qualquer solução de (2.30). Seja $c(s)=(\alpha(s), \rho(s), \sigma(s))$ a solução de (2.30) com valores iniciais $c(0)=\left(1,0, \frac{\pi}{2}\right.$ ) (vide Figura 2.9). Assim $I \equiv 1$ ao longo de $c$. Portanto, $\sigma(s) \in(0, \pi)$, para todo $s \geq 0$. Da terceira equação em (2.30) segue que $\frac{d \sigma}{d s}(s)>0$ se $s>0$. Então $\sigma$ é estritamente crescente e limitada superiormente, $\operatorname{com} \sigma(s) \in\left(\frac{\pi}{2}, \pi\right]$, para $s>0$. Usando de novo a terceira equação em (2.30), temos que

$$
0=\lim _{s \rightarrow+\infty} \frac{d \sigma}{d s}=(4 n+2) \sin \lim _{s \rightarrow+\infty} \sigma(s) .
$$

Isto é $\lim _{s \rightarrow+\infty} \sigma(s)=\pi$. Assim, da primeira equação em (2.30), $\alpha$ decresce monotonicamente e anula-se no infinito.

Como $I \equiv 1$ ao longo de $c$, temos que $\sin \sigma=\alpha^{2 n+1}$. Na outra mão, das duas primeiras equações em (2.30) tem-se $\frac{d \rho}{d \alpha}=\frac{\tan \sigma}{2 \sqrt{\alpha}}$. Portanto, obtemos

$$
\rho(\alpha)=\frac{1}{2} \int_{\alpha}^{1} \sqrt{\frac{t^{4 n+1}}{1-t^{4 n+2}}} d t,
$$

para $0<\alpha \leq 1$. Esta é uma integral elíptica convergente em $t=1$. Enfim, construímos uma solução completa $\gamma$ de (2.30) refletindo na reta $\rho=0$ (vide Observação 2.35 (i)). Esta por sua vez gera uma hipersuperfície $\Gamma$ de $\mathbf{H}_{\mathbf{Q}}^{n}$ difeomorfa a $\mathbf{R}^{4 n-1}$ cuja fronteira ideal $\partial \Gamma$ é o fecho da reunião do par de órbitas correspondentes a os pontos $(0, \pm R)$, onde $R=\rho(0)$. No modelo de Siegel temos que

$$
\partial \Gamma=\left\{\zeta \in \partial \mathscr{S}^{n}: \Re\left(\zeta_{n-1}\right)=R\right\} \cup\left\{\zeta_{\infty}\right\} \cup\left\{\zeta \in \partial \mathscr{S}^{n}: \Re\left(\zeta_{n-1}\right)=-R\right\}
$$

Assim $\partial \Gamma$ é homeomorfa a um buquê de duas esferas $\mathbf{S}^{4 n-2}$ grudadas no ponto $\zeta_{\infty}$. Finalmente usando as dilatações da Observação 2.35(ii) obtemos a folheação desejada. Isto conclui a demonstração do item (ii) do Teorema 0.4 .

(iii) Da Observação 2.35(i) e (iii) temos que as retas $\rho=t, t \in \mathbf{R}$ são soluções de (2.30), que constituem uma folheação do espaço das órbitas invariante pelas translações em $\rho$, cujas correspondentes hipersuperfícies geradas $\Gamma_{t}$ são fans. Assim $\left(\Gamma_{t}\right)_{t \in \mathbf{R}}$ é uma folheação de $\mathbf{H}_{\mathbf{Q}}^{n}$ invariante pelo grupo a um parâmetro de transvecções induzido pelas translações em $\rho$. O restante da prova segue da Proposição 2.22. Isto completa a demonstração do item (iii) do Teorema 0.4 . 


\section{CAPÍTULO 3}

\section{Hipersuperfícies mínimas do plano projetivo octoniônico}

No caso dos espaços simétricos não compactos $G / K$, usamos a decomposição de Iwasawa $G=N A K$ do seu grupo de isometrias $G$, para encontrar subgrupos $H \subset G$ que atuam sobre $G / K$ com cohomogeneidade dois. No presente capítulo estudaremos o caso compacto, a saber construiremos hipersuperfícies mínimas do plano projetivo octoniônico $\mathbf{P}_{\mathbf{O}}^{2}=F_{4} / \operatorname{Spin}(9)$. Em [PT99] foram classificados os subgrupos de $F_{4}$ que agem sobre $\mathbf{P}_{\mathbf{O}}^{2}$ polarmente com cohomogeneidade dois, a saber $H=\operatorname{Spin}(8), T^{1} \cdot \operatorname{Spin}(7), S U(2) \cdot S U(4), S U(3) \cdot S U(3)$. O caso $H=\operatorname{Spin}(8)$ foi abordado em [Tom96] (assim como os grupos correspondentes para $\mathbf{P}_{\mathbf{C}}^{n}$ e $\left.\mathbf{P}_{\mathbf{Q}}^{n}\right)$. Nós estudamos os casos $H=T^{1} \cdot \operatorname{Spin}(7)$ e $S U(2) \cdot S U(4)$. O caso $H=S U(3) \cdot S U(3)$ requer métodos diferentes dos usados aqui e preferimos abordá-lo em um trabalho futuro.

Na primeira seção expomos as propriedades básicas da álgebra dos octônions e lembramos o Principio de trialidade. Na segunda seção estudamos a álgebra de Jordan. No terceiro capítulo construímos o modelo de Veronese para $\mathbf{P}_{\mathbf{O}}^{2}$. Na quarta e última seção estudamos as ações polares de $H=T^{1} \cdot \operatorname{Spin}(7), S U(2) \cdot S U(4)$ sobre o plano projetivo octoniônico. Os conceitos básicos acerca das propriedades algébricas dos octônions foram tomados de [Ada96], [CS03], [Whi78] e [CR98].

\subsection{A álgebra dos octônions $\mathrm{O}$}

\subsubsection{O}

É um fato bem conhecido (Teorema de Hurwitz) que a única álgebra normada real de dimensão 8 é a álgebra não associativa dos octônions $\mathbf{O}$. Seja $\{1, i, j, k, l, i l, j l, k l\}$ a base ortonormal can̂onica de $\mathbf{O}$ em relação ao produto interno

$$
\langle a, b\rangle=\Re(a \bar{b})
$$


para todo par $a, b \in \mathbf{O}$. Temos a isometria $\mathbf{Q} \oplus \mathbf{Q}=\mathbf{O}$, dada por $(p, q) \mapsto p+q l$, onde $(p, q) \cdot\left(p^{\prime}, q^{\prime}\right)=:\left(p p^{\prime}-\overline{q^{\prime}} q, q^{\prime} p+q \overline{p^{\prime}}\right), \overline{(p, q)}=:(\bar{p},-q),\left\langle(p, q),\left(p^{\prime}, q^{\prime}\right)\right\rangle=:\left\langle p, p^{\prime}\right\rangle+\left\langle q, q^{\prime}\right\rangle$, para todo par $p, q \in \mathbf{Q}$ (vide $\S 2.3 .1$ ).

Proposição 3.1. (1) $i(j l)=-k l$, logo $\mathbf{O}$ é não associativa

(2) para todo par $a, b \in \mathbf{O}, a(b l)=(b a) l$

(3) para todo par $p, q \in \mathbf{Q}$, tem-se $(p l) q=(p \bar{q}) l$

(4) para todo par $a, b \in \mathbf{O}$ temos que

(i) $\Re(a b)=\Re(b a)$ e $(a b)=-\Im(b a)$, se a,b são imaginários puros

(ii) $a \bar{a}=|a|^{2}=\bar{a} a$

(iii) $\langle a, b\rangle=\langle\bar{a}, \bar{b}\rangle$

(iv) $\bar{a}(a b)=(\bar{a} a) b e(a b) \bar{b}=a(b \bar{b})$.

Definição 3.2. O associador de $a, b, c \in \mathbf{O}$ é dado por $[a, b, c]=:(a b) c-a(b c)$.

Proposição 3.3. Dados $a, b, c \in \mathbf{O}$, temos que o associador $[a, b, c]$

(i) é imaginário puro

(ii) muda se sinal se conjugarmos qualquer variável

(iii) é uma funcão alternada das três variáveis

(iv) em particular as partes reais de $(a b) c,(b c) a,(c a) b, a(b c), b(c a)$ e $c(a b)$, são iguais.

Para uma demonstração das proposições 3.1 e 3.3 acima, vide [Ada96] cap.15.

Proposição 3.4 (Identidades de Moufang (vide [CS03])). Dados a, b, c $\in \mathbf{O}$ tem-se

(i) $(a b)(c a)=(a(b c)) a=a((b c) a)$. Em particular $a(b a)=(a b)$ a e portanto faz sentido a expressão aba.

(ii) $a(b(a c))=(a b a) c$

(iii) $((b a) c) a=b(a c a)$

Seja $G_{2}$ o grupo $\operatorname{Aut}(\mathbf{O})$ dos automorfismos de $\mathbf{O}$, a saber

$$
G_{2}=\{g \in \mathbf{G L}(\mathbf{O}): g(a b)=g(a) g(b) \text { para todo par } a, b \in \mathbf{O}\}
$$

Proposição 3.5. $G_{2}$ é o grupo de Lie compacto excepcional de dimensão 14.

A prova deste fato está contida na demonstração do Corolário 3.11 em §3.1.2. 


\subsubsection{Princípio de trialidade}

Consideremos $\mathbf{O}$ com a estrutura de espaço euclidiano de dimensão 8 usual, i.e. a métrica é dada por

$$
\langle a, b\rangle=\Re(a \bar{b})
$$

para todo par $a, b \in \mathbf{O}$.

Dado $\xi \in \mathbf{S}^{7}$, denotemos a reflexão de $\mathbf{O}$ com respeito ao hiperplano $\xi^{\perp}$ por $\rho_{\xi}$. Então temos que

$$
\rho_{\xi} a=-\xi \bar{a} \xi
$$

para todo $a \in \mathrm{O}$. Note que, o membro direito da expressão acima faz sentido, vide Proposição 3.4(i). As translações à esquerda e direita $L_{\xi}$ e $R_{\xi}$, estão dadas por

$$
L_{\xi} a=\xi a \text { e } R_{\xi} a=a \xi, a \in \mathbf{O}
$$

Note que, da Proposição 3.1(4)(iii), temos que a conjugação $a \mapsto \bar{a}$ é um elemento de $O(8)$. De fato, esta é a reflexão de $\mathbf{O}$ em relação a 1. Na outra mão, das proposições 3.3(iv) e $3.1(4)\left(\right.$ iv), segue $L_{\xi}, R_{\xi}$ e $\rho_{\xi}$ são elementos de $O(8)$. Portanto, as reflexões $\rho_{\xi}$ pertencem a $O(8)$.

Como sabemos os elementos de $S O(8)$ são produtos de uma quantidade par de reflexões. Consideremos $h_{1} \in S O(8)$. Sem perda de generalidade, suponhamos que $h_{1}=\rho_{\xi} \rho_{\eta}$, com $\xi, \eta \in \mathbf{S}^{7}$. Então para todo par $a, b \in \mathbf{O}$ da Proposição 3.4(i) temos que

$$
\begin{aligned}
h_{1}(a b) & =\rho_{\xi}\left(\rho_{\eta} a b\right) \\
& =\xi(\overline{\eta(\overline{a b}) \eta}) \xi \\
& =(\xi(\bar{\eta} a))((b \bar{\eta}) \xi) \\
& =h_{2}(a) h_{3}(b),
\end{aligned}
$$

onde $h_{2}=L_{\xi} L_{\bar{\eta}}$ e $h_{3}=R_{\xi} R_{\bar{\eta}}$. Assim obtemos o chamado Princípio de trialidade: para todo $h_{1} \in S O(8)$ existem $h_{2}, h_{3} \in S O(8)$, tais que

$$
h_{1}(a b)=h_{2}(a) h_{3}(b), \text { para todo par } a, b \in \mathbf{O}
$$

onde $h_{2}$ e $h_{3}$ são únicos a menos de mudança comum de sinais.

Definição 3.6. Denotamos com $\operatorname{Spin}(8)$ o subgrupo de $S O(8) \times S O(8) \times S O(8)$ de todas as triplas que satisfazem $(T)$.

Observação 3.7. (i) Note que Spin(8) é um subgrupo fechado de $S O(8) \times S O(8) \times S O(8)$. Portanto é um grupo de Lie Compacto. Na outra mão, da construção em (3.2) e da conexidade de $\mathbf{S}^{7}$ segue que $\operatorname{Spin}(8)$ é conexo. 
(ii) Nosso objetivo seguinte é justificar a Definição 3.6, a saber mostrar que a projeção $\operatorname{Spin}(8) \rightarrow S O(8), h \mapsto h_{1}$ é o recobrimento universal de $S O(8)$.

Definição 3.8. Consideremos os seguintes subgrupos de $\operatorname{Spin}(8)$ :

(i) $\operatorname{Spin}^{\alpha}(7)=\left\{h \in \operatorname{Spin}(8): h_{\alpha} 1=1\right\}$, para $\alpha=1,2,3$

(ii) $G_{2}=\operatorname{Spin}^{1}(7) \cap \operatorname{Spin}^{2}(7) \cap \operatorname{Spin}^{3}(7)$

(iii) $\operatorname{Spin}(6)=\left\{h \in \operatorname{Spin}^{1}(7): h_{1} i=i\right\}$

(iv) $\operatorname{Spin}(5)=\left\{h \in \operatorname{Spin}(6): h_{1} j=j\right\}$.

Note que, todos os grupos na definição 3.8 são compactos, pois são subgrupos fechados de $\operatorname{Spin}(8)$.

Observação 3.9. (i) Temos que, (T) é equivalente a

$$
h_{2}(a b)=h_{1}(a) \tilde{h}_{3}(b) \quad\left(T^{\prime}\right) \text { e } h_{3}(a b)=\tilde{h}_{2}(a) h_{1}(b) \quad\left(T^{\prime \prime}\right)
$$

onde $\tilde{h}_{\alpha}(a)=: \overline{h_{\alpha}(\bar{a})}$. Logo, as 'permutações' $\left(h_{1}, h_{2}, h_{3}\right) \mapsto\left(h_{2}, h_{1}, \tilde{h}_{3}\right)$ e $\left(h_{1}, h_{2}, h_{3}\right) \mapsto$ $\left(h_{3}, \tilde{h}_{2}, h_{1}\right)$, são automorfismos de $\operatorname{Spin}(8)$ que permutam os $\operatorname{Spin}^{\alpha}(7)$.

(ii) Em particular, $h \in \operatorname{Spin}(8)$ pertence a $\operatorname{Spin}^{1}(7)\left(\right.$ resp. $\operatorname{Spin}^{2}(7)$ e $\left.\operatorname{Spin}^{3}(7)\right)$ se e somente se $h_{3}=\tilde{h}_{2}\left(\right.$ resp. $h_{2}=h_{1}$ e $\left.h_{2}=h_{1}\right)$.

(iii) $G_{2}$ é o grupo dos automorfismos de $\mathbf{O}$.

(iv) Suponhamos que $h_{1}, h_{2}, h_{3} \in O(8)$ satisfazem $(T)$. Então $\left(h_{1}, h_{2}, h_{3}\right) \in \operatorname{Spin}(8)$. Com efeito. Suponhamos que $h_{1} \notin S O(8)$. Então, existe $g_{1} \in S O(8)$ tal que $g_{1} h_{1} a=\bar{a}$, para todo $a \in \mathbf{O}$. Sejam $g_{2}, g_{3} \in S O(8)$ tais que $\left(g_{1}, g_{2}, g_{3}\right)$ satisfaz $(T)$. Se $d=g h$, temos que $d_{1} 1=1$, logo por $\left(T^{\prime \prime}\right) d_{3}=\tilde{d}_{2}$. Assim $\bar{a}=d_{1}(a)=d_{2}(a) \tilde{d}_{2}(1)$, e portanto, $\bar{a} d_{2} 1=d_{2} a$, para todo $a \in \mathbf{O}$. Isto implica que $\overline{a b}=\bar{a} \bar{b}$ para todo par $a, b \in \mathbf{O}$, o qual é um absurdo.

Proposição 3.10. Temos os seguintes isomorfismos de grupos de Lie

(i) $S U(4)=\operatorname{Spin}(6)$

(ii) $S U(3)=\left\{g \in \operatorname{Spin}(6): g_{2} 1=1\right\}$

(iii) $\operatorname{Sp}(2)=\operatorname{Spin}(5)$. 
Demonstração. Considere a estrutura complexa sobre $\mathbf{O}$ dada por $L_{i}$. Se $h=\left(h_{1}, h_{2}, h_{3}\right) \in$ $\operatorname{Spin}(6)$, da Definição 3.8(iii) e das observações 3.9(ii) e (i), temos que para $a \in \mathbf{O}$ tem-se

$$
i h_{2}(a)=h_{1}(i) h_{2}(a)=h_{1}(i) \tilde{h}_{3}(a)=h_{2}(i a)
$$

Por tanto $h_{2} \in S O(8)$ e comuta com $L_{i}$. Na outra mão $h_{2}=$ id se e somente se $h=$ (id, id, id). Assim a aplicação $h \mapsto h_{2}$ define o isomorfismo desejado. Isto mostra o item (i). O item (ii) é conseqüência direta de (i). Enfim, o item (iii) segue analogamente a (i) vendo que $h_{2}$ comuta com a estrutura quaterniônica de $\mathbf{O}$ dada por $L_{i}, L_{j}$ e $L_{k}=L_{i} L_{j}$.

Corolário 3.11. Todos os grupos na Definição 3.8 são simplesmente conexos.

Demonstração. Da Proposição 3.10 temos que $\operatorname{Spin}(5)$ e Spin(6) são simplesmente conexos. Agora, $\operatorname{Spin}(7)$ age transitivamente sobre a esfera $\mathbf{S}^{6} \subset \Im(\mathbf{O})$, com isotropia $\operatorname{Spin}(6)$, assim obtemos o fibrado $\operatorname{Spin}(6) \hookrightarrow \operatorname{Spin}^{1}(7) \rightarrow \mathbf{S}^{6}$. Portanto, $\operatorname{Spin}^{1}(7)$ e simplesmente conexo. Da Observação 3.9(i) segue que $\operatorname{Spin}^{2}(7)$ e $\operatorname{Spin}^{2}(7)$ também são simplesmente conexos. Na outra mão, $\operatorname{Spin}^{1}(7)$ atua transitivamente sobre $\mathbf{S}^{7}$ com isotropia $G_{2}$. Então $G_{2}$ é simplesmente conexo e tem dimesão 14.

Note que, $\operatorname{Spin}(8)$ atua transitivamente sobre $\mathbf{S}^{7}$ com isotropia $\operatorname{Spin}^{1}(7)$. Então $\operatorname{Spin}(8)$ é simplesmente conexo. Ainda mais, a projeção $h \mapsto h_{1}$, define um recobrimento duplo de $\operatorname{Spin}(8)$ sobre $S O(8)$. A restrição deste aos subgrupos na Definição 3.8(i), (iii) e (iv), define os correspondentes recobrimentos duplos. Na outra mão, temos que $\operatorname{Spin}^{\alpha}(7), \alpha=1,2$, 3, são três cópias não congruentes de $\operatorname{Spin}(7)$ dentro de $\operatorname{Spin}(8)$, pois os automorfismos na Observação 3.9(i) são externos (vide [CR98]).

\subsection{A álgebra de Jordan}

Consideremos o espaço vetorial (real) $\mathscr{J}=\left\{x \in \mathscr{M}(3, \mathbf{O}): x^{*}=x\right\}$, das matrizes hermitianas de ordem três com coeficientes nos octônions, munido com o produto

$$
x \circ y=\frac{1}{2}(x y+x y)
$$

onde o produto no membro direito é o produto usual de matrizes. Dizemos que $\mathscr{J}$ é uma álgebra de Jordan. Note que $x \in \mathscr{J}$ se e somente se

$$
x=\left[\begin{array}{lll}
a_{1} & x_{1} & x_{2} \\
\overline{x_{1}} & a_{2} & \overline{x_{3}} \\
\overline{x_{2}} & x_{3} & a_{3}
\end{array}\right],
$$

onde $a_{1}, a_{2}, a_{3} \in \mathbf{R}$ e $x_{1}, x_{2}, x_{3} \in \mathbf{O}$. Segue que $\mathscr{J}$ é isomorfo, como espaço vetorial real, a $\mathbf{R}^{27}$. Assim se $x=\left(a_{\alpha}, x_{\alpha}\right), y=\left(b_{\alpha}, y_{\alpha}\right), z=\left(c_{\alpha}, z_{\alpha}\right) \in \mathscr{J}$ com $z=x \circ y$ combinando (3.3) 
com (3.4) obtemos

$$
\begin{aligned}
& c_{1}=a_{1} b_{1}+\Re\left(x_{1} \overline{y_{1}}+x_{2} \overline{y_{2}}\right) \\
& c_{2}=a_{2} b_{2}+\Re\left(\overline{x_{1}} y_{1}+\overline{x_{3}} y_{3}\right) \\
& c_{3}=a_{3} b_{3}+\Re\left(\overline{x_{2}} y_{2}+x_{3} \overline{y_{3}}\right)
\end{aligned}
$$

$\mathrm{e}$

$$
\begin{aligned}
& z_{1}=\frac{1}{2}\left\{\left(b_{1}+b_{2}\right) x_{1}+\left(a_{1}+a_{2}\right) y_{2}+x_{2} y_{3}+y_{2} x_{3}\right\} \\
& z_{2}=\frac{1}{2}\left\{\left(b_{1}+b_{3}\right) x_{2}+\left(a_{1}+a_{3}\right) y_{2}+x_{1} \overline{y_{3}}+y_{1} \overline{x_{3}}\right\} \\
& z_{3}=\frac{1}{2}\left\{\left(b_{2}+b_{3}\right) x_{3}+\left(a_{2}+a_{3}\right) y_{3}+\overline{x_{2}} y_{1}+\overline{y_{2}} x_{1}\right\}
\end{aligned}
$$

Sobre $\mathscr{J}$ definimos a métrica euclidiana $\langle\cdot, \cdot\rangle$ dada por

$$
\langle x, y\rangle=\frac{1}{2} \operatorname{tr}(x \circ y)
$$

para todo par $x, y \in \mathscr{J}$. Das equações (3.5) e (3.7) segue que

$$
\langle x, y\rangle=\frac{1}{2}\left(a_{1} b_{1}+a_{2} b_{2}+a_{3} b_{3}\right)+\Re\left(x_{1} \overline{y_{1}}+x_{2} \overline{y_{2}}+x_{3} \overline{y_{3}}\right) .
$$

\subsubsection{O grupo $F_{4}$}

Seja $F_{4}$ o grupo $\operatorname{Aut}(\mathscr{J})$ dos automorfismos de $\mathscr{J}$, a saber

$$
F_{4}=\{f \in \mathbf{G L}(\mathscr{J}): f(x \circ y)=(f x) \circ(f y), \text { para todo par } x, y \in \mathscr{J}\}
$$

Seja $G=\left\{f \in F_{4}: \operatorname{tr}(f x)=\operatorname{tr}(x)\right.$ para todo $\left.x \in \mathscr{J}\right\}$. Então $G$ é um subgrupo fechado de $O(27)$ e, portanto, compacto.

Lema 3.12 (Vide [Yok68]§3). Para todo $x \in \mathscr{J}$ existe $g \in G$ tal que gx é diagonal.

Corolário 3.13. (i) O funcional $\mathbf{R}$-linear $x \mapsto \operatorname{tr}(x), x \in \mathscr{J}$, é invariante pelo grupo $F_{4}$. Em particular $F_{4}$ é um subgrupo compacto de $O(27)$.

(ii) Podemos trocar $G$ por $F_{4}$ no Lema 3.12.

Sejam $e_{1}, e_{2}$ e $e_{3}$ os elementos de $\mathscr{J}$ seguintes:

$$
e_{1}=\left[\begin{array}{lll}
1 & 0 & 0 \\
0 & 0 & 0 \\
0 & 0 & 0
\end{array}\right], e_{2}=\left[\begin{array}{lll}
0 & 0 & 0 \\
0 & 1 & 0 \\
0 & 0 & 0
\end{array}\right], e_{3}=\left[\begin{array}{lll}
0 & 0 & 0 \\
0 & 0 & 0 \\
0 & 0 & 1
\end{array}\right] .
$$


Demonstração do Corolário 3.13. Seja $f \in F_{4}$ então $\operatorname{tr}\left(f e_{\alpha}\right)=1$, para $i=1,2$, 3. Com efeito, pelo Lema 3.12 podemos considerar $f e_{\alpha}=b_{1} e_{1}+b_{2} e_{2}+b_{3} e_{3}$. Como $e_{\alpha}$ é idempotente, então $f e_{\alpha}$ também. $\operatorname{Logo} b_{\beta}^{2}=b_{\beta}$ para $\beta=1,2,3$. Na outra mão, segue facilmente das equações (3.5) e (3.6) que $e_{\alpha}$ não é suma de idempotentes não triviais. Portanto o mesmo acontece com $f e_{\alpha}$, assim $\left\{b_{1}, b_{2}, b_{3}\right\}=\{0,1\}$. Agora, dado $x \in \mathscr{J}$, seja $g \in G$ tal que $g x=b_{1} e_{1}+b_{2} e_{2}+b_{3} e_{3}$. Então se $f \in F_{4}$ temos que

$$
\begin{aligned}
\operatorname{tr}(f x) & =b_{1} \operatorname{tr}\left(f g^{-1} e_{1}\right)+b_{2} \operatorname{tr}\left(f g^{-1} e_{2}\right)+b_{3} \operatorname{tr}\left(f g^{-1} e_{3}\right) \\
& =b_{1}+b_{2}+b_{3} \\
& =\operatorname{tr}(g x) \\
& =\operatorname{tr}(x) .
\end{aligned}
$$

Isto junto com a equação (3.7) mostra o item (i). O item (ii) segue diretamente de (i).

\subsubsection{O grupo $\operatorname{Spin}(9)$}

Seja $\mathscr{J}_{0}=\{x \in \mathscr{J}: \operatorname{tr}(x)=0\}$. Consideremos os elementos $e_{1}, e_{2}, e_{3} \in \mathscr{J}_{0}$ definidos em (3.8). Sejam $\operatorname{Spin}^{\alpha}(9)=\left\{f \in F_{4}: f e_{\alpha}=e_{\alpha}\right\}, \alpha=1,2,3$, e $H=\operatorname{Spin}^{1}(9) \cap \operatorname{Spin}^{2}(9) \cap$ $\operatorname{Spin}^{3}(9)$. Temos que $\operatorname{Spin}^{\alpha}(9)$, e $H$ são subgrupos fechados de $F_{4}$, para $\alpha=1,2,3$. Portanto são compactos. Consideremos os subespaços $V_{\alpha}=\left\{x \in \mathscr{J}_{0}: e_{\alpha} \circ x=0\right\}, \alpha=1,2,3$. Usando as equações (3.5) e (3.6) vemos que estes são isométricos (como espaços euclidianos) a $\mathbf{R}^{9}$.

Lema 3.14. O grupo $H$ é isomorfo a Spin(8).

Demonstração. Com efeito, sejam $W_{\alpha}=\left\{x \in V_{\alpha}: x\right.$ tem diagonal nula $\}$, para $\alpha=1,2,3$. Dado $h \in H$ sejam $h_{\alpha}=\left.h\right|_{W_{\alpha}}$. Assim para cada $x \in \mathscr{J}$ temos que

$$
h(x)=\left[\begin{array}{ccc}
a_{1} & h_{1} x_{1} & h_{2} x_{2} \\
\overline{h_{1} x_{1}} & a_{2} & \overline{h_{3} x_{3}} \\
\overline{h_{2} x_{2}} & h_{3} x_{3} & a_{3}
\end{array}\right],
$$

onde $\left(h_{1}, h_{2}, h_{2}\right)$ satisfaz o Principio de trialidade $(T)$, pois $h(x \circ y)=(h x) \circ(h y) \in W_{3}$, para $x \in W_{2}, y \in W_{1}$ e cada $h \in H$. Da Observação $3.9\left(\right.$ vi) segue que $\left(h_{1}, h_{2}, h_{3}\right) \in \operatorname{Spin}(8)$.

Consideremos as trasnformações $\mathbf{R}$-lineares $f_{1}, f_{2}, f_{3}$ dadas por

$$
x=\left[\begin{array}{lll}
a_{1} & x_{1} & x_{2} \\
\overline{x_{1}} & a_{2} & \overline{x_{3}} \\
\overline{x_{2}} & x_{3} & a_{3}
\end{array}\right] \mapsto\left[\begin{array}{lll}
a_{1} & x_{2} & x_{1} \\
\overline{x_{2}} & a_{3} & x_{3} \\
\overline{x_{1}} & \overline{x_{3}} & a_{2}
\end{array}\right],\left[\begin{array}{lll}
a_{3} & x_{3} & \overline{x_{2}} \\
\overline{x_{3}} & a_{2} & \overline{x_{1}} \\
x_{2} & x_{1} & a_{1}
\end{array}\right] \text { e }\left[\begin{array}{ccc}
a_{2} & \overline{x_{1}} & \overline{x_{3}} \\
x_{1} & a_{1} & x_{2} \\
x_{3} & \overline{x_{2}} & a_{3}
\end{array}\right],
$$

respectivamente. Temos que $f_{1}, f_{2}$ e $f_{3}$ são elementos de $F_{4}$ tais que $f_{\alpha} e_{\alpha}=e_{\alpha}$ e $f_{\alpha} e_{\beta}=e_{\gamma}$, para $\alpha, \beta$ e $\gamma$ disjuntos dois a dois.

Lema 3.15. Consideremos a esfera unitária $\mathbf{S}^{8} \subset V_{3}$. Então 
(i) $\operatorname{Spin}^{3}(9)$ age transitivamente sobre $\mathbf{S}^{8}$ e o subgrupo de isotropia de $e_{1}-e_{2}$ é $H$. Em particular $\operatorname{Spin}^{3}(9)$ é simplesmente conexo.

(ii) A aplicação $\operatorname{Spin}^{3}(9) \rightarrow S O(9)$ induzida pela restrição dos elementos de $\operatorname{Spin}^{3}(9) \subset F_{4}$ a $V_{3}$ é um recobrimento duplo.

Demonstração. Para uma prova da primeira afirmação no item (i) vide [Yok68]§6. Agora, seja $e=e_{1}+e_{2}+e_{3}$ a matriz identidade. Dado $f \in F_{4}$ temos que $f e=e$. Em particular, se $f \in \operatorname{Spin}^{3}(9)$ tem-se $f\left(e_{1}+e_{2}\right)=e_{1}+e_{2}$. Logo, se $f \in \operatorname{Spin}^{3}(9)$ fixa $e_{1}-e_{2} \in \mathbf{S}^{7}$, então $f \in H$. Portanto obtemos o fibrado $\operatorname{Spin}(8) \hookrightarrow \operatorname{Spin}^{3}(9) \rightarrow \mathbf{S}^{8}$. Logo, $\operatorname{Spin}^{3}(9)$ é simplesmente conexo. Para mostrar o item (ii) seja $p: \operatorname{Spin}^{3}(9) \rightarrow S O(9)$, onde $p(f)$ restrição de $f$ a $V_{3}$. Suponhamos que $f v=v$ para todo $v \in V_{3}$. Em particular, $f \in H$. Assim, a correspondente tripla $\left(h_{1}, h_{2}, h_{3}\right)$ na demonstração do Lema 3.14 , pertence, pelo Principio de trialidade $(T)$, a $\{(\mathrm{id}, \pm \mathrm{id}, \pm \mathrm{id})\}$. Logo, $p$ é um recobrimento duplo.

Observação 3.16. Note que os grupos $\operatorname{Spin}^{\alpha}(9), \alpha=1,2$, 3, são três cópias de $\operatorname{Spin}(9)$ dentro de $F_{4}$ cuja intersecção é $\operatorname{Spin}(8)$. De fato, são todos conjugados entre si pelos elementos $f_{1}, f_{2}, f_{3} \in F_{4}$ definidos em (3.10). Em diante $\operatorname{Spin}(9)$ denotará o grupo $\operatorname{Spin}^{3}(9)$.

\subsection{Modelo de Veronese}

A álgebra com divisão e não comutativa dos octônions $\mathbf{O}$ é, por sua vez, não associativa. Isto constitui um impedimento para adotar coordenadas homogêneas para o espaço projetivo octoniônico. No entanto, existe uma maneira de mergulhá-lo isometricamente na esfera $\mathbf{S}^{26}\left(\frac{1}{\sqrt{2}}\right)$. A idéia é devida a Freudenthal [Fre85] e pode ser estendia a $\mathbf{P}_{\mathbf{R}}^{n}, \mathbf{P}_{\mathbf{C}}^{n}$ e $\mathbf{P}_{\mathbf{Q}}^{n}$. Para entendê-la basta pensar no caso real. A uma reta $\ell \subset \mathbf{R}^{n+1}$ dada, podemos associalhe (biunivocamente) uma matriz simétrica $L$, tal que $L^{2}=L$ e $\operatorname{tr}(L)=1$. A matriz $L$ representa a projeção ortogonal de $\mathbf{R}^{n+1}$ sobre $\ell$. Este mergulho é conhecido também como o modelo de Veronese para o plano projetivo octoniônico (em geral para os espaços projetivos). Usaremos este modelo para fazer nossos cômputos seguindo algumas idéias de [Iwa81].

Seja $M=\left\{x \in \mathscr{J}: x^{2}=x \operatorname{e~} \operatorname{tr}(x)=1\right\}$. Então, das equações (3.5) e (3.6), $x \in M$ se somente se

$$
\begin{aligned}
& a_{1}=a_{1}^{2}+\left|x_{1}\right|^{2}+\left|x_{2}\right|^{2} \\
& a_{2}=a_{2}^{2}+\left|x_{1}\right|^{2}+\left|x_{3}\right|^{2} \\
& a_{3}=a_{3}^{2}+\left|x_{2}\right|^{2}+\left|x_{3}\right|^{2}
\end{aligned}
$$




$$
\begin{gathered}
x_{1}=\left(a_{1}+a_{2}\right) x_{1}+x_{2} x_{3} \\
x_{2}=\left(a_{1}+a_{3}\right) x_{2}+x_{1} \overline{x_{3}} \\
x_{3}=\left(a_{2}+a_{3}\right) x_{3}+\overline{x_{2}} x_{1} \\
1=a_{1}+a_{2}+a_{3} .
\end{gathered}
$$

Observação 3.17. Note que as equações (3.11), (3.12) e (3.13) descrevem cartas locais para $M$ via o Teorema do posto ([Rud64] Teo. 9.20), vide a demonstração da Proposição 3.20(i) abaixo. Portanto $M$ é uma subvariedade mergulhada de $\mathscr{J} \simeq \mathbf{R}^{27}$ (vide equação (3.4)). Ainda mais, das equações (3.7), (3.13) e da definição de $M$ segue que $M$ está contida na intersecção da esfera $\mathbf{S}^{26}\left(\frac{1}{\sqrt{2}}\right)$ com um hiperplano de $\mathbf{R}^{27}$.

Lema 3.18. Dado $x \in M$, então existe $f \in F_{4}$ tal que $f x=e_{3}$. Em particular $F_{4}$ atua transitivamente por isometrias sobre $M$ e o subgrupo de isotropia de e é Spin(9).

Demonstração. Seja $x \in M$. Então, pelo Corolário 3.13(ii), existe $f \in F_{4}$ tal que $f x=$ $b_{1} e_{1}+b_{2} e_{2}+b_{3} e_{3}$. Como $x$ é idempotente e de traço um, segue que $b_{1}+b_{2}+b_{3}=b_{1}^{2}+b_{2}^{2}+b_{3}^{2}=$ $b_{1}^{3}+b_{2}^{3}+b_{3}^{3}=1$. Portanto $\left\{b_{1}, b_{2}, b_{3}\right\}=\{0,1\}$. A primeira afirmação segue de compor $f$, se necessário, com um automorfismo apropriado dos definidos em (3.10). A segunda afirmação segue trivialmente da primeira junto com a Observação 3.16.

Como corolário imediato do Lema 3.18 temos:

Proposição 3.19. $M$ é isométrico a $\mathbf{P}_{\mathbf{O}}^{2}$.

Proposição 3.20. Escolhendo $p=e_{3}$ como ponto base de $M$, temos que

(i) O espaço tangente a $M$ em $p$ é

$$
T_{p} M=\left\{p \circ x: x \in \mathscr{J}_{0}\right\}=\left\{\left[\begin{array}{ccc}
0 & 0 & u \\
0 & 0 & v \\
\bar{u} & \bar{v} & 0
\end{array}\right]: u, v \in \mathbf{O}\right\}=\mathbf{O}^{2} .
$$

(ii) A exponencial riemanniana em $p$ é dada por

$$
\operatorname{Exp}\left[\begin{array}{l}
u \\
v
\end{array}\right]=\left[\begin{array}{ccc}
\frac{\sin ^{2} r}{r^{2}}|u|^{2} & \frac{\sin ^{2} r}{r^{2}} u \bar{v} & \frac{\sin 2 r}{2 r} u \\
\frac{\sin ^{2} r}{r^{2}} v \bar{u} & \frac{\sin ^{2} r}{r^{2}}|v|^{2} & \frac{\sin 2 r}{2 r} v \\
\frac{\sin 2 r}{2 r} \bar{u} & \frac{\sin 2 r}{2 r} \bar{v} & \cos ^{2} r
\end{array}\right],
$$

para todo $\left[\begin{array}{l}u \\ v\end{array}\right] \in \mathbf{O}^{2}$, onde $r=\sqrt{|u|^{2}+|v|^{2}}$. 
(iii) As esferas métricas de raio $r$ com centro em $p$ estão dadas por

$$
S(r)=\left\{x \in M: a_{3}=\cos ^{2} r\right\},
$$

para $r \in\left(0, \frac{\pi}{2}\right)$.

(iv) O cut locus de p é o conjunto

$$
\operatorname{Cut}(p)=\left\{x \in M: a_{3}=0\right\}
$$

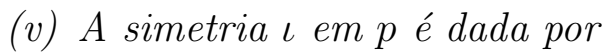

$$
x=\left[\begin{array}{lll}
a_{1} & x_{1} & x_{2} \\
\overline{x_{1}} & a_{2} & \overline{x_{3}} \\
\overline{x_{2}} & x_{3} & a_{3}
\end{array}\right] \mapsto \iota(x)=\left[\begin{array}{ccc}
a_{1} & x_{1} & -x_{2} \\
\overline{x_{1}} & a_{2} & -\overline{x_{3}} \\
\overline{x_{2}} & -x_{3} & a_{3}
\end{array}\right] .
$$

Demonstração. Consideremos a aplicação

$$
\begin{aligned}
\phi: \mathscr{J} & \rightarrow \mathscr{J} \\
x & \mapsto x^{2}-x .
\end{aligned}
$$

Assim $d \phi_{x} X=X x+x X-X$, para todo $x \in \mathscr{J}$ e cada $X \in T_{x} \mathscr{J}=\mathscr{J}$. Em particular $d \phi_{p} X=0$ se e somente se

$$
X=\left[\begin{array}{lll}
0 & 0 & u \\
0 & 0 & v \\
\bar{u} & \bar{v} & 0
\end{array}\right]
$$

para $u, v \in \mathbf{O}$. Por outro lado, $\phi$ é $F_{4}$-equivariante. Portanto o item (i) segue do Teorema do posto.

Agora, seja $\psi: \mathbf{O}^{2} \rightarrow M$ a aplicação dada por

$$
\psi\left(\left[\begin{array}{l}
u \\
v
\end{array}\right]\right)=\left[\begin{array}{ccc}
\frac{\sin ^{2} r}{r^{2}}|u|^{2} & \frac{\sin ^{2} r}{r^{2}} u \bar{v} & \frac{\sin 2 r}{2 r} u \\
\frac{\sin ^{2} r}{r^{2}} v \bar{u} & \frac{\sin ^{2} r}{r^{2}}|v|^{2} & \frac{\sin 2 r}{2 r} v \\
\frac{\sin 2 r}{2 r} \bar{u} & \frac{\sin 2 r}{2 r} \bar{v} & \cos ^{2} r
\end{array}\right],
$$

para todo $\left[\begin{array}{l}u \\ v\end{array}\right] \in \mathbf{O}^{2}$, onde $r=\sqrt{|u|^{2}+|v|^{2}}$. Note que, por (3.11), (3.12) e (3.13) $\psi$ está 
bem definida. Fixemos $\left[\begin{array}{l}u \\ v\end{array}\right] \in \mathbf{O}^{2}$ e consideremos a curva $\gamma(t)=\psi\left(t\left[\begin{array}{l}u \\ v\end{array}\right]\right)$. Temos que

$$
\frac{d \gamma}{d t}=\left[\begin{array}{ccc}
\frac{\sin (2 r t)}{r}|u|^{2} & \frac{\sin (2 r t)}{r} u \bar{v} & \cos (2 r t) u \\
\frac{\sin (2 r t)}{r} v \bar{u} & \frac{\sin (2 r t)}{r}|v|^{2} & \cos (2 r t) v \\
\cos (2 r t) \bar{u} & \cos (2 r t) \bar{v} & -r \sin (2 r t)
\end{array}\right]
$$

para todo $t \in \mathbf{R}$. Portanto $\left\langle\frac{d \gamma}{d t}, \frac{d \gamma}{d t}\right\rangle \equiv r^{2}$. Assim, pela teoria geral de espaços simétricos compactos de posto um (vide [Gor10]), $\gamma$ minimiza a distância entre $p$ e $\gamma(t)$. Isto mostra o item (ii). Os ítens (iii) e (iv) seguem diretamente de (ii). Por último, um cálculo direto mostra que $\iota$ é um elemento de $F_{4}$ tal que $\iota^{2}$ é a identidade em $M, d \iota_{p}$ é menos a identidade em $T_{p} M$ e o único ponto fixo de $\iota$ em $M-\operatorname{Cut}(p)$ é $p$.

Corolário 3.21. Cut $(p)$ é difeomorfo à esfera $\mathbf{S}^{8}$.

Demonstração. Com efeito, da Proposição 3.20(iii) e das equações (3.11), (3.12) e (3.13), segue que $x \in \operatorname{Cut}(p)$ se e somente se $x=\frac{1}{2}\left[\begin{array}{ccc}1+t & u & 0 \\ \bar{u} & 1-t & 0 \\ 0 & 0 & 0\end{array}\right]$, onde $u \in \mathbf{O}$ e $t \in \mathbf{R}$, com $|u|^{2}+t^{2}=1$

Note que a ação isotrópica $\left(\operatorname{Spin}(9), T_{p} M\right)$ é dada pela restrição de $\left(F_{4}, \mathscr{J}\right)$. Com efeito, seja $y \in T_{p} M$, então $y=p \circ x \operatorname{com} \operatorname{tr}(x)=0$. Logo, se $f \in \operatorname{Spin}(9)$, então $\operatorname{tr}(f x)=0$ e $f y=p \circ f x$. Consideremos a álgebra de Lie $\mathfrak{F}_{4}$ de $F_{4}$, isto é

$$
\mathfrak{F}_{4}=\{X \in \mathbf{G L}(\mathscr{J}): X(x \circ y)=X(x) \circ y+x \circ X(y) \text {, para todo par } x, y \in \mathscr{J}\}
$$

Observação 3.22. (i) Note que, a álgebra de Lie de $\operatorname{Spin}(9)$ é $\mathfrak{S O}(9)=\left\{X \in \mathfrak{F}_{4}: X p=\right.$ $0\}$, e a ação $\left(\mathfrak{S O}(9), T_{p} M\right)$ é dada pela restrição de $\left(\mathfrak{F}_{4}, \mathscr{J}\right)$.

(ii) Do Princípio de trialidade $(T)$, temos o Princípio de trialidade infinitesimal, a saber, dado $X_{1} \in \mathfrak{S O}(8)$ existem $X_{2}, X_{3} \in \mathfrak{S O}(8)$, tais que

$$
X_{1}(a b)=\left(X_{2} a\right) b+a\left(X_{3} b\right), \text { para todo par } a, b \in \mathbf{O}
$$

Portanto, a álgebra de Lie $\mathfrak{S O}(8)$ de $\operatorname{Spin}(8)$ é o conjunto das triplas em $\mathfrak{S O O}(8) \times$ $\mathfrak{S O}(8) \times \mathfrak{S O}(8)$ que satisfazem $(T I)$. Em particular, as álgebras de Lie dos grupos $\operatorname{Spin}^{\alpha}(7)$ são: $\mathfrak{S O}^{\alpha}(7)=\left\{X \in \mathfrak{S O}(8): X_{\alpha} 1=0\right\}$, para $\alpha=1,2,3$.

(iii) Se $A \in S O(3), A$ induz o elemento $f_{A} \in F_{4}$ dado por $x \mapsto A x A^{-1}$ (produto de matrizes de ordem três). Em particular obtemos o mergulho $S O(3) \rightarrow F_{4} . A \in S O(3)$ representa um elemento de $\operatorname{Spin}(9)$ se e somente se $A=\left[\begin{array}{ccc}\cos t & \sin t & 0 \\ -\sin t & \cos t & 0 \\ 0 & 0 & 1\end{array}\right]$. 
(iv) Analogamente $S U(3)$ pode ser mergulhado em $F_{4}$. Para ver isto, é mais fácil pensar no nível das álgebras de Lie. Neste caso, a afirmação segue comprovando diretamente que $\mathfrak{S} \mathfrak{U}(3)$ atua sobre $\mathscr{J}$ por derivações assim: $x \mapsto X x-x X$ (produto de matrizes de ordem três), para todo $x \in \mathscr{J}$ e cada $X \in \mathfrak{S U}(3)$. Em particular, $A \in S U(3)$ representa um elemento de $\operatorname{Spin}(9)$ se e somente se $A=\left[\begin{array}{ccc}\lambda & \mu & 0 \\ -\bar{\mu} & \lambda & 0 \\ 0 & 0 & 1\end{array}\right]$, onde $\lambda, \mu \in \mathbf{C}$ $\operatorname{com}|\lambda|^{2}+|\mu|^{2}=1$

\subsection{Hipersuperfícies mínimas}

Nesta seção estudaremos as ações dos subgrupos $H=T^{1} \cdot \operatorname{Spin}(7), S U(2) \cdot S U(4)$ de $\operatorname{Spin}(9)$ sobre o plano projetivo octoniônico $\mathbf{P}_{\mathbf{O}}^{2}$. Estas são ações polares e suas seções são isométricas ao plano projetivo real $\mathbf{P}_{\mathbf{R}}^{2}$. Para fazer nossos cômputos usaremos as ações slice correspondentes (vide Definição 1.1). De fato $H$ fixa o ponto base $p, \log \nu_{p} H(p)=T_{p} M$. Construiremos primeiro seções $\tilde{\Sigma}$ para $\left(H, T_{p} M\right)$ e notaremos que $\Sigma=\operatorname{Exp}(\tilde{\Sigma})$ é uma seção $\operatorname{para}\left(H, \mathbf{P}_{\mathbf{O}}^{2}\right)$.

\subsubsection{Raízes de $\mathrm{P}_{\mathrm{O}}^{2}$}

Se $G / K$ é um espaço simétrico, então o par $(K, G / K)$ é hiperpolar (vide [Gor10]). Consideremos a decomposição ortogonal $\mathfrak{G}=\mathfrak{K}+\mathfrak{M}$, onde $\mathfrak{M}$ se identifica com o espaço tangente a $G / K$ no ponto base. Seja $\mathfrak{A}$ um subespaço abeliano maximal de $\mathfrak{M}$. Neste caso, $\operatorname{Exp}(\mathfrak{A})$ (exponencial riemanniana) é uma seção para $(K, G / K)$, assim a cohomogeneidade é o posto de $G / K$. Em particular, se $G / K$ tem posto um, temos que qualquer geodésica radial passando pelo ponto base é uma seção.

Por outra parte, se $G / K$ é um espaço simétrico de tipo compacto, como é bem conhecido, tem-se uma decomposição da álgebra de Lie $\mathfrak{G}$ de $G$, com respeito às raízes restritas, análoga à descrita em $\S 2.2 .1$ para o caso não compacto (vide [Loo69]). Em particular, se $G / K=\mathbf{P}_{\mathbf{C}}^{n}$, $\mathbf{P}_{\mathbf{Q}}^{n}, \mathbf{P}_{\mathbf{O}}^{2}$ (posto um), têm-se exatamente duas raízes restritas. Denotamos as raízes restritas de $\mathbf{P}_{\mathbf{O}}^{2} \operatorname{com} \lambda$ e $2 \lambda$.

Continuemos usando a notação de $\S 3.3$.

Seja $\xi=\left[\begin{array}{l}0 \\ 1\end{array}\right] \in T_{p} \mathbf{P}_{\mathbf{O}}^{2}$ e consideremos a geodésica radial $\gamma(r)=\operatorname{Exp}(r \xi)$. Então os tipos de isotropia e órbita de $\left(\operatorname{Spin}(9), \mathbf{P}_{\mathbf{O}}^{2}\right)$ são:

$$
\begin{cases}\operatorname{Spin}(9) \text { e }\{p\}, & \text { se } r=0 \\ \operatorname{Spin}^{3}(7) \text { e } S(r) \approx \mathbf{S}^{15}(\text { esferas métricas de raio } r), & \text { se } r=\in\left(0, \frac{\pi}{2}\right) \\ \operatorname{Spin}(8) \text { e } \operatorname{Cut}(p) \approx \mathbf{S}^{8}, & \text { se } r=\frac{\pi}{2}\end{cases}
$$


Temos que $\mathfrak{F}_{4}=\mathfrak{S O}(9)+\mathfrak{M}\left(\mathfrak{M} \approx T_{p} \mathbf{P}_{\mathbf{O}}^{2}\right)$. Sejam $X \in \mathfrak{M}$ tal que $X_{p}^{*}=\xi$ e $\mathfrak{A}=\mathbf{R} X$. Agora, consideremos a decomposição de $\mathfrak{S O}(9)$ relativa à órbita $\operatorname{Cut}(p)=\operatorname{Spin}(9) / \operatorname{Spin}(8)$ é

$$
\mathfrak{S O}(9)=\mathfrak{S O}(8)+\mathfrak{P}
$$

onde $\mathfrak{P}$ é identificado com o espaço tangente a $\operatorname{Cut}(p)$ em $q=\gamma\left(\frac{\pi}{2}\right)$. A saber (vide Corolário $3.21)$

$$
T_{q} \operatorname{Cut}(p)=\left\{\left[\begin{array}{lll}
0 & u & 0 \\
\bar{u} & 0 & 0 \\
0 & 0 & 0
\end{array}\right]: u \in \mathbf{O}\right\} .
$$

Temos que $\mathfrak{P}$ corresponde aos campos de Jacobi ao longo de geodésicas radiais unindo $p$ e $q$ que se anulam unicamente em $p$. Portanto $[X, \mathfrak{P}]=\mathfrak{M}_{\lambda}$. Da observação 3.22(iii) segue que o grupo a um parâmentro $r \mapsto \exp (r X)$ (exponencial de Lie) é representado pelas matrizes $\left[\begin{array}{ccc}1 & 0 & 0 \\ 0 & \cos r & \sin r \\ 0 & -\sin r & \cos r\end{array}\right], r \in \mathbf{R} . \quad$ Assim $\exp \left(-\frac{\pi}{2} X\right)_{*} \mathfrak{P}=\mathfrak{M}_{\lambda} . \quad$ Ou seja $\mathfrak{M}_{\lambda} \approx$ $\left\{\left[\begin{array}{l}u \\ v\end{array}\right] \in \mathbf{O}^{2}: v=0\right\}$. Por outro lado, a decomposição de $\mathfrak{S} \mathfrak{O}(9)$ relativa à órbita $S(1)=$ $\operatorname{Spin}(9) / \operatorname{Spin}(8)$ é

$$
\mathfrak{S O}(9)=\mathfrak{S O}^{3}(7)+\mathfrak{Q}
$$

onde $\mathfrak{Q}$ se identifica com o espaço tangente a $S(1)$ em $\gamma(1)$. Da observação 3.22(ii) segue que $[Z, X]=0$ para todo $Z \in \mathfrak{S O}^{3}(7)$. Logo $\mathfrak{S O}^{3}(7)$ é o centralizador de $X$ em $\operatorname{Spin}(9)$ e, portanto, $\mathfrak{M}_{2 \lambda} \approx\left\{\left[\begin{array}{l}u \\ v\end{array}\right] \in \mathbf{O}^{2}: u=0\right.$ e $\left.v \in \Im(\mathbf{O})\right\}$. Assim obtemos as decomposições:

$$
\mathfrak{S O}(9)=\mathfrak{K}_{0}+\mathfrak{K}_{\lambda}+\mathfrak{K}_{2 \lambda} \text { e } \mathfrak{M}=\mathfrak{A}+\mathfrak{M}_{\lambda}+\mathfrak{M}_{2 \lambda}
$$

onde $\mathfrak{K}_{0}=\mathfrak{S O}^{3}(7), \mathfrak{K}_{\lambda}=\left[\mathfrak{M}_{\lambda}, X\right]$ e $\mathfrak{K}_{2 \lambda}=\left[\mathfrak{M}_{2 \lambda}, X\right]$.

\subsubsection{Geometria de $\operatorname{Spin}(8), T^{1} \cdot \operatorname{Spin}(7)$ e $S U(2) \cdot S U(4)$}

Do ponto de vista geométrico, os grupos $\operatorname{Spin}(8), T^{1} \cdot \operatorname{Spin}(7)$ e $S U(2) \cdot S U(3)$ podem ser definidos como sendo os estabilizadores em $F_{4}$ dos seguintes flags (vide [PT99]): $(q, \operatorname{Cut}(p))$, $(\beta, \operatorname{Cut}(p))$ e $\left(\mathbf{S}^{2}, \operatorname{Cut}(p)\right)$, respectivamente, onde $q, \beta$ e $\mathbf{S}^{2}$ são um ponto, uma geodésica e uma esfera totalmente geodésica em $\operatorname{Cut}(p)$. Com efeito, consideremos os pontos $e_{1}, e_{2}$, $e_{3} \in \mathbf{P}_{\mathbf{O}}^{2}$ dados por (3.8). Temos que $p=e_{3}$. Seja $q=e_{2} \in \operatorname{Cut}(p)$. Se um subgrupo de $F_{4}$ estabiliza $\operatorname{Cut}(p)$, então está contido em $\operatorname{Spin}(9)$. Logo, se ademais fixa $q$, então, como todo elemento de $F_{4}$ fixa $e=e_{1}+e_{2}+e_{3}$, temos que tal subgrupo fixa $e_{1}$. Assim coincide com $\operatorname{Spin}(8)$. Agora consideremos a curva $\beta(t)=\left[\begin{array}{ccc}\sin ^{2} t & \frac{1}{2} \sin 2 t & 0 \\ \frac{1}{2} \sin 2 t & \cos ^{2} t & 0 \\ 0 & 0 & 0\end{array}\right]$. Usando o elemento $f_{1}$ 
de $F_{4}$ definido em (3.10) junto com (3.14), vemos que $\beta$ é uma geodésica em $\operatorname{Cut}(p)$ ligando $q$ e $e_{1}$. Seu estabilizador é $T^{1} \cdot \operatorname{Spin}^{1}(7)$, onde $T^{1}$ é representado pelo grupo a um parâmetro $t \mapsto\left[\begin{array}{ccc}\cos t & \sin t & 0 \\ -\sin t & \cos t & 0 \\ 0 & 0 & 1\end{array}\right], t \in \mathbf{R}$, vide Observação 3.22(iii). Analogamente, da Observação 3.22(iv) e da Proposição 3.22(ii), segue que o estabilizador da esfera $\mathbf{S}^{2}=\{(z, t) \in \mathbf{C} \times \mathbf{R}$ : $\left.|z|^{2}+t^{1}=1\right\} \rightarrow \operatorname{Cut}(p)$ (vide Corolário 3.21) é $S U(2) \cdot S U(3)$.

Na outra mão, vide [PT99] os grupos $H=\operatorname{Spin}(8), T^{1} \cdot \operatorname{Spin}(7)$ e $S U(2) \cdot S U(4)$ atuam sobre $\mathbf{P}_{\mathbf{O}}^{2}$ com cohomogeneidade dois e as correspondentes ações isotrópicas $\left(H, \mathbf{O}^{2}\right)$ no ponto base $p$ são equivalentes à soma de $\left(\operatorname{Spin}^{2}(7), \mathbf{O}\right)$ e $\left(\operatorname{Spin}^{3}(7), \mathbf{O}\right),\left(S O(2) \times S O(8), \mathbf{R}^{2} \otimes \mathbf{R}^{8}\right)$ e $\left(S U(2) \times S U(4), \mathbf{C}^{2} \otimes_{\mathbf{R}} \mathbf{C}^{4}\right)$, respectivamente. Temos que:

Lema 3.23. Consideremos os grupos $H=\operatorname{Spin}(8), T^{1} \cdot \operatorname{Spin}(7)$ e $S U(2) \cdot S U(4)$. Sejam $\tilde{S}(1)$ a esfera unitária em $T_{p} \mathbf{P}_{\mathbf{O}}^{2}$ e $S(r)$ a esfera métrica com centro em $p$ e de raio $r$ em $\mathbf{P}_{\mathbf{O}}^{2}$. Então a transformação

$$
\begin{aligned}
\psi: \tilde{S}(1) & \rightarrow S_{r} \\
z & \mapsto \operatorname{Exp}(r z),
\end{aligned}
$$

é um difeomorfismo $H$-invariante tal que $\mathscr{V}\left(H(\psi(z))=2^{-7}(\sin r)^{7}(\sin 2 r)^{7} \tilde{\mathscr{V}}(H(z))\right.$.

Observação 3.24. Não é difícil ver que o par $\left(\operatorname{Spin}(8), \mathbf{P}_{\mathbf{O}}^{2}\right)$ é polar e (vide [Tom96]) $\phi(r, \theta)=$ $\operatorname{Exp}\left[\begin{array}{l}r \sin \theta \\ r \cos \theta\end{array}\right],(r, \theta) \in\left[0, \frac{\pi}{2}\right] \times\left[0, \frac{\pi}{2}\right]$, parametriza o espaço das órbitas $\operatorname{Spin}(8) \backslash \mathbf{P}_{\mathbf{O}}^{2}$, o qual é um triângulo geodésico em $\mathbf{S}^{2}$ com todos os ângulos retos. Além disso, os tipos de isotropia e órbita são

$$
\begin{cases}\operatorname{Spin}(8) \text { e um ponto, } & \text { nos vértices } \\ \operatorname{Spin}^{\alpha}(7)(\alpha=1,2,3) \text { e } \mathbf{S}^{7}, & \text { nas arestas } \\ G_{2} \text { e } \mathbf{S}^{7} \times \mathbf{S}^{7}, & \text { no interior }\end{cases}
$$

a) $T^{1} \cdot \operatorname{Spin}(7)$

Neste parágrafo $\operatorname{Spin}(7)$ denotará o grupo $\operatorname{Spin}^{3}(7)$ (vide Definição 3.8). Pela Observação 3.22(iii), os elementos de $T^{1} \subset \operatorname{Spin}(9)$ são representados por matrizes da forma

$$
R_{t}=\left[\begin{array}{ccc}
\cos t & \sin t & 0 \\
-\sin t & \cos t & 0 \\
0 & 0 & 1
\end{array}\right]
$$

com $t \in \mathbf{R}$. Seja $H=T^{1} \times \operatorname{Spin}(7)$. Assim, dados $h=\left(R_{t}, g\right) \in H$ e $x \in M$, temos que

$$
h(x)=R_{t} g(x) R_{t}^{-1},
$$


onde $g(x)$ é dado pela equação (3.9).

Observação 3.25. Note que $H$ não atua efetivamente, mas o quociente de $H$ por um subgrupo finito sim, a saber $T^{1} \cdot \operatorname{Spin}(7)=T^{1} \times \operatorname{Spin}^{\times}(7) / \mathbf{Z}_{2}$, onde

$$
\mathbf{Z}_{2}=\left\{\left(R_{0},(\mathrm{id}, \mathrm{id}, \mathrm{id})\right),\left(R_{\pi},(\mathrm{id},-\mathrm{id},-\mathrm{id})\right\}\right.
$$

Temos que a ação slice em $\nu_{p} H(p)=T_{p} M=\mathbf{O}^{2}$ é dada por

$$
h\left[\begin{array}{l}
u \\
v
\end{array}\right]=\left[\begin{array}{l}
g_{2}(\cos t u+\sin t v) \\
g_{2}(\cos t v-\sin t u)
\end{array}\right]
$$

para todo $\left[\begin{array}{l}u \\ v\end{array}\right] \in \mathbf{O}^{2}$ e cada $h \in H$. Portanto, $\tilde{\Sigma}=\left\{\left[\begin{array}{l}u \\ v\end{array}\right] \in \mathbf{O}^{2}: u \in \mathbf{R}\right.$ e $\left.v \in i \mathbf{R}\right\}$ é uma seção para $\left(H, \mathbf{O}^{2}\right)$. Identificando $\tilde{\Sigma}$ com $\mathbf{R}^{2}$ temos que o grupo de Weyl $W_{\tilde{\Sigma}}$ é gerado pelas reflexões nas retas $x=0, y=0, x=y$ e $x=-y$. Assim, o espaço das órbitas $H \backslash \mathbf{O}^{2}$ é

$$
\tilde{\Delta}=\left\{(x, y) \in \mathbf{R}^{2}: x, y, x-y \geqq 0\right\} .
$$

Temos que $\Sigma=\operatorname{Exp}(\tilde{\Sigma}) \simeq \mathbf{P}_{\mathbf{R}}^{2}$ é uma seção para $\left(H, \mathbf{P}_{\mathbf{O}}^{2}\right)$. Se $\phi(r, \theta)=\operatorname{Exp}\left[\begin{array}{c}i r \sin \theta \\ r \cos \theta\end{array}\right]$, de (3.14) obtemos

$$
\phi(r, \theta)=\left[\begin{array}{ccc}
\sin ^{2} r \sin ^{2} \theta & \frac{i}{2} \sin ^{2} r \sin 2 \theta & \frac{i}{2} \sin 2 r \sin \theta \\
-\frac{i}{2} \sin ^{2} r \sin 2 \theta & \sin ^{2} r \cos ^{2} \theta & \frac{1}{2} \sin 2 r \cos \theta \\
-\frac{i}{2} \sin 2 r \sin \theta & \frac{1}{2} \sin 2 r \cos \theta & \cos ^{2} r
\end{array}\right]
$$

Então $\Sigma=\{\phi(r, \theta): r \geqq 0$ e $\theta \in \mathbf{R}\}$. Em particular, o espaço das órbitas $H \backslash \mathbf{P}_{\mathbf{O}}^{2}$ é dado por

$$
\Delta=\left\{\phi(r, \theta): r \in\left[0 \frac{\pi}{2}\right] \text { e } \theta \in\left[0, \frac{\pi}{4}\right]\right\}
$$

Logo, os tipos de isotropia e órbita de $\left(H, \mathbf{P}_{\mathbf{O}}^{2}\right)$ são

$$
H_{\phi(r, \theta)}= \begin{cases}H, & \text { se } r=0 \\ \mathbf{Z}_{2} \times \operatorname{Spin}^{\times}(7), & \text { se } r=\frac{\pi}{2} \text { e } \theta=0 \\ T^{1} \times S U(4), & \text { se } r=\frac{\pi}{2} \text { e } \theta=\frac{\pi}{4} \\ \mathbf{Z}_{2} \times S U(4), & \text { se } r=\frac{\pi}{2} \text { e } \theta \in\left(0, \frac{\pi}{4}\right) \\ U(3), & \text { se } r \in\left(0, \frac{\pi}{2}\right) \text { e } \theta=\frac{\pi}{4} \\ \mathbf{Z}_{2} \times G_{2}, & \text { se } r \in\left(0, \frac{\pi}{2}\right) \text { e } \theta=0 \\ \mathbf{Z}_{2} \times S U(3), & \text { se } r \in\left(0, \frac{\pi}{2}\right) \text { e } \theta \in\left(0, \frac{\pi}{4}\right)\end{cases}
$$


e

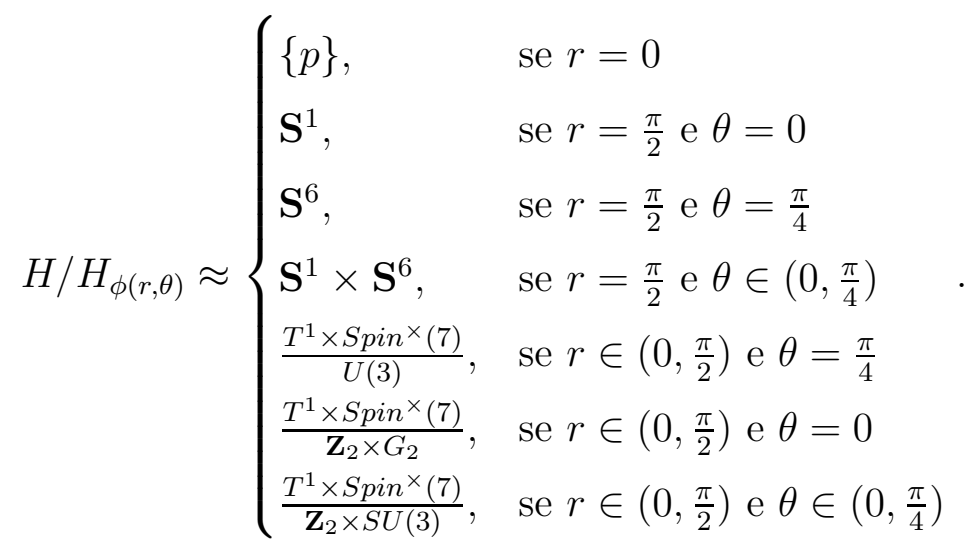

Onde

$$
\begin{aligned}
U(3) & \rightarrow T^{1} \times \operatorname{Spin}^{\times}(7) \\
B & \mapsto\left(R_{t}, g\right),
\end{aligned}
$$

com $\operatorname{det}(B)=\cos t+i \sin t$ e $g \in S U(4), g_{2}=\left[\begin{array}{cc}\operatorname{det}(B)^{-1} & 0 \\ 0 & B\end{array}\right]$

$$
\begin{aligned}
\mathbf{Z}_{2} \times G_{2} & \rightarrow T^{1} \times \operatorname{Spin}^{\times}(7) \\
( \pm 1, B) & \mapsto\left(R_{t}, g\right),
\end{aligned}
$$

$\operatorname{com} \cos t= \pm 1$ e $g=(\cos t B, \cos t B, \cos t B)$.

$$
\begin{aligned}
\mathbf{Z}_{2} \times S U(3) & \rightarrow T^{1} \times \operatorname{Spin}^{\times}(7) \\
( \pm 1, B) & \mapsto\left(R_{t}, g\right)
\end{aligned}
$$

$\operatorname{com} \cos t= \pm 1$ e $g \in S U(4), g_{2}=\left[\begin{array}{cc}\cos t & 0 \\ 0 & \cos t B\end{array}\right]$.

Temos que, o funcional de volume no ponto $(x, y) \in \tilde{\Delta}$ é dado por $\tilde{\mathscr{V}}(x, y)=(x y)^{6}\left(x^{2}-\right.$ $\left.y^{2}\right)$. A métrica orbital e o funcional de volume (vide Lema 3.23) no ponto $\phi(r, \theta) \in \Delta$ são dados por

$$
\begin{aligned}
d s^{2} & =d r^{2}+\sin ^{2} r d \theta^{2}, \\
\mathscr{V}(r, \theta) & =(\sin r)^{7}(\sin 2 r)^{7}(\sin 2 \theta)^{6} \cos 2 \theta .
\end{aligned}
$$

Portanto, do Teorema 1.18 obtemos

Proposição 3.26. Sejam $\gamma(s)=(r(s), \theta(s))$ uma curva em $\Delta$ parametrizada pelo comprimento de arco s (em relação à métrica orbital $d s^{2}$ ) e $\sigma$ o ângulo entre $\frac{\partial}{\partial r}$ e $\frac{d \gamma}{d s}$. Então a 
hipersuperfície $\Gamma$ de $\mathbf{P}_{\mathbf{O}}^{2}$, com $H \backslash \Gamma=\gamma$, tem curvatura média $\mathbf{h}$ se e somente se

$$
\begin{aligned}
& \frac{d r}{d s}=\cos \sigma \\
& \frac{d \theta}{d s}=\frac{\sin \sigma}{\sin r} \\
& \frac{d \sigma}{d s}=(12 \cot 2 \theta-2 \tan 2 \theta) \frac{\cos \sigma}{\sin r}-(15 \cot r-7 \tan r) \sin \sigma+\mathbf{h}
\end{aligned}
$$

Observação 3.27. (i) Note que, no bordo $\partial \Delta=\left\{(r, \theta): \theta=0\right.$ ou $\left.\theta=\frac{\pi}{2}\right\}$, o sistema (3.16) é singular. Porém, podemos considerar soluções saindo ortogonalmente de $\partial \Delta$ (vide Lema 0.6).

(ii) Soluções explícitas. Note que se $\mathbf{h} \equiv \pm(15 \cot a-7 \tan a)$, então $r=a$ é uma solução de (3.16) que gera uma esfera métrica de raio $a$ com centro no ponto base $p$. Em particular a solução $r=\arctan \sqrt{\frac{15}{7}}$ gera uma esfera mínima, a saber a esfera métrica de volume máximo. Na outra mão $\theta=\frac{1}{2} \arctan \sqrt{6}$ é uma solução de (3.16) para $\mathbf{h} \equiv 0$ que gera uma hipersuperfície singular (no ponto base) homeomorfa à reunião (de espaços topológicos, identificando as fronteiras) de um cone sobre a órbita principal $\frac{T^{1} \times \operatorname{Spin}^{\times}(7)}{\mathbf{Z}_{2} \times S U(3)}$ e a aplicação cilíndrica do fibrado canônico (vide Observação 1.5(i))

$$
\mathbf{S}^{7} \hookrightarrow \frac{T^{1} \times \operatorname{Spin}^{\times}(7)}{\mathbf{Z}_{2} \times S U(3)} \longrightarrow \mathbf{S}^{1} \times \mathbf{S}^{6}
$$

b) $S U(2) \cdot S U(4)$

Pela Observação 3.22(iv), os elementos de $S U(2) \subset \operatorname{Spin}(9)$ são representados por matrizes da forma

$$
R_{\lambda, \mu}=\left[\begin{array}{ccc}
\lambda & \mu & 0 \\
-\bar{\mu} & \bar{\lambda} & 0 \\
0 & 0 & 1
\end{array}\right]
$$

$\operatorname{com} \lambda, \mu \in \mathbf{C ~ e ~}|\lambda|^{2}+|\mu|^{2}=1$. Seja $H=S U(2) \times S U(4)$. Assim, dados $h=\left(R_{\lambda, \mu}, g\right) \in H$ e $x \in M$ temos que

$$
h(x)=R_{\lambda, \mu} g(x) R_{\lambda, \mu}^{-1},
$$

onde $g(x)$ é dado pela equação (3.9).

Observação 3.28. Note que $H$ não atua efetivamente, mas o quociente de $H$ por um subgrupo finito sim, a saber $S U(2) \cdot S U(4)=S U(2) \times S(4) / \mathbf{Z}_{2}$, onde

$$
\mathbf{Z}_{2}=\left\{\left(R_{1,0},(\mathrm{id}, \mathrm{id}, \mathrm{id})\right),\left(R_{-1,0},(\mathrm{id},-\mathrm{id}-\mathrm{id})\right)\right\}
$$


Temos que a ação slice em $\nu_{p} H(p)=T_{p} M=\mathbf{O}^{2}$ é dada por

$$
h\left[\begin{array}{l}
u \\
v
\end{array}\right]=\left[\begin{array}{l}
g_{2}(\lambda u+\mu v) \\
g_{2}(\bar{\lambda} v-\bar{\mu} u)
\end{array}\right]
$$

para todo $\left[\begin{array}{l}u \\ v\end{array}\right] \in \mathbf{O}^{2}$ e cada $h \in H$. Portanto, $\tilde{\Sigma}=\left\{\left[\begin{array}{l}u \\ v\end{array}\right] \in \mathbf{O}^{2}: u \in \mathbf{R}\right.$ e $\left.v \in j \mathbf{R}\right\}$ é uma seção para $\left(H, \mathbf{O}^{2}\right)$. Identificando $\tilde{\Sigma}$ com $\mathbf{R}^{2}$ temos que o grupo de Weyl $W_{\tilde{\Sigma}}$ é gerado pelas reflexões nas retas $x=0, y=0, x=y$ e $x=-y$. Assim, o espaço das órbitas $H \backslash \mathbf{O}^{2}$ é

$$
\tilde{\Delta}=\left\{(x, y) \in \mathbf{R}^{2}: x, y, x-y \geqq 0\right\} .
$$

Temos que $\Sigma=\operatorname{Exp}(\tilde{\Sigma}) \simeq \mathbf{P}_{\mathbf{R}}^{2}$ é uma seção para $\left(H, \mathbf{P}_{\mathbf{O}}^{2}\right)$. Se $\phi(r, \theta)=\operatorname{Exp}\left[\begin{array}{c}j r \sin \theta \\ r \cos \theta\end{array}\right]$, de (3.14) obtemos

$$
\phi(r, \theta)=\left[\begin{array}{ccc}
\sin ^{2} r \sin ^{2} \theta & \frac{j}{2} \sin ^{2} r \sin 2 \theta & \frac{j}{2} \sin 2 r \sin \theta \\
-\frac{j}{2} \sin ^{2} r \sin 2 \theta & \sin ^{2} r \cos ^{2} \theta & \frac{1}{2} \sin 2 r \cos \theta \\
-\frac{j}{2} \sin 2 r \sin \theta & \frac{1}{2} \sin 2 r \cos \theta & \cos ^{2} r
\end{array}\right]
$$

Então $\Sigma=\{\phi(r, \theta): r \geqq 0$ e $\theta \in \mathbf{R}\}$. Em particular, o espaço das órbitas $H \backslash \mathbf{P}_{\mathbf{O}}^{2}$ é dado por

$$
\Delta=\left\{\phi(r, \theta): r \in\left[0 \frac{\pi}{2}\right] \text { e } \theta \in\left[0, \frac{\pi}{4}\right]\right\}
$$

Logo, os tipos de isotropia e órbita de $\left(H, \mathbf{P}_{\mathbf{O}}^{2}\right)$ são

$$
H_{\phi(r, \theta)}= \begin{cases}H, & \text { se } r=0 \\ U(1) \times S U(4), & \text { se } r=\frac{\pi}{2} \text { e } \theta=0 \\ S U(2) \times S p(2), & \text { se } r=\frac{\pi}{2} \text { e } \theta=\frac{\pi}{4} \\ U(1) \times S p(2), & \text { se } r=\frac{\pi}{2} \text { e } \theta \in\left(0, \frac{\pi}{4}\right) \\ S U(2), & \text { se } r \in\left(0, \frac{\pi}{2}\right) \text { e } \theta=\frac{\pi}{4} \\ U(3), & \text { se } r \in\left(0, \frac{\pi}{2}\right) \text { e } \theta=0 \\ U(1) \times S U(2), & \text { se } r \in\left(0, \frac{\pi}{2}\right) \text { e } \theta \in\left(0, \frac{\pi}{4}\right)\end{cases}
$$


e

$$
H / H_{\phi(r, \theta)} \approx\left\{\begin{array}{ll}
\{p\}, & \text { se } r=0 \\
\mathbf{S}^{2}, & \text { se } r=\frac{\pi}{2} \text { e } \theta=0 \\
\mathbf{S}^{5}, & \text { se } r=\frac{\pi}{2} \text { e } \theta=\frac{\pi}{4} \\
\mathbf{S}^{2} \times \mathbf{S}^{5}, & \text { se } r=\frac{\pi}{2} \text { e } \theta \in\left(0, \frac{\pi}{4}\right) \\
\frac{S U(2) \times S U(4)}{S U(2)}, & \text { se } r \in\left(0, \frac{\pi}{2}\right) \text { e } \theta=\frac{\pi}{4} \\
\frac{S U(2) \times S U(4)}{U(3)}, & \text { se } r \in\left(0, \frac{\pi}{2}\right) \text { e } \theta=0 \\
\frac{S U(2) \times S U(4)}{U(1) \times S U(2)}, & \text { se } r \in\left(0, \frac{\pi}{2}\right) \text { e } \theta \in\left(0, \frac{\pi}{4}\right)
\end{array} .\right.
$$

Onde

$$
\begin{aligned}
S U(2) & \rightarrow S U(2) \times S U(4) \\
R_{\lambda, \mu} & \mapsto\left(R_{\lambda, \mu}, g\right),
\end{aligned}
$$

$\operatorname{com} g \in S p(2), g_{2}=\left[\begin{array}{cc}\bar{\lambda}-\mu j & 0 \\ 0 & \bar{\mu}+\lambda j\end{array}\right]$

$$
\begin{aligned}
U(3) & \rightarrow S U(2) \times S U(4) \\
B & \mapsto\left(R_{\lambda, 0}, g\right),
\end{aligned}
$$

$$
\begin{gathered}
\operatorname{com} g_{2}=\left[\begin{array}{cccc}
b_{11} & 0 & b_{12} & b_{13} \\
0 & \lambda & 0 & 0 \\
b_{12} & 0 & b_{22} & b_{23} \\
b_{31} & 0 & b_{32} & b_{33}
\end{array}\right], \text { onde } B=\left(b_{\alpha, \beta}\right) \text { e } \lambda=\operatorname{det}(B) . \\
U(1) \times S U(2) \rightarrow S U(2) \times S U(4) \\
(\lambda, B) \mapsto\left(R_{\lambda, 0}, g\right),
\end{gathered}
$$

com $g_{2}=\left[\begin{array}{ccc}\bar{\lambda} & 0 & 0 \\ 0 & \lambda & 0 \\ 0 & 0 & B\end{array}\right]$. Temos que, o funcional de volume no ponto $(x, y) \in \tilde{\Delta}$ é dado por $\tilde{\mathscr{V}}(x, y)=(x y)^{5}\left(x^{2}-y^{2}\right)^{2}$. A métrica orbital e o funcional de volume (vide Lema 3.23) no ponto $\phi(r, \theta) \in \Delta$ são dados por

$$
\begin{aligned}
d s^{2} & =d r^{2}+\sin ^{2} r d \theta^{2}, \\
\mathscr{V}(r, \theta) & =(\sin r)^{7}(\sin 2 r)^{7}(\sin 2 \theta)^{5}(\cos 2 \theta)^{2} .
\end{aligned}
$$

Portanto, do Teorema 1.18 obtemos

Proposição 3.29. Sejam $\gamma(s)=(r(s), \theta(s))$ uma curva em $\Delta$ parametrizada pelo compri- 
mento de arco $s$ (em relação à métrica orbital $d s^{2}$ ) e $\sigma$ o ângulo entre $\frac{\partial}{\partial r}$ e $\frac{d \gamma}{d s}$. Então a hipersuperfície $\Gamma$ de $\mathbf{P}_{\mathbf{O}}^{2}$, com $H \backslash \Gamma=\gamma$, tem curvatura média $\mathbf{h}$ se e somente se

$$
\begin{aligned}
& \frac{d r}{d s}=\cos \sigma \\
& \frac{d \theta}{d s}=\frac{\sin \sigma}{\sin r} \\
& \frac{d \sigma}{d s}=(10 \cot 2 \theta-4 \tan 2 \theta) \frac{\cos \sigma}{\sin r}-(15 \cot r-7 \tan r) \sin \sigma+\mathbf{h} .
\end{aligned}
$$

Observação 3.30. (i) Note que, no bordo $\partial \Delta=\left\{(r, \theta): \theta=0\right.$ ou $\left.\theta=\frac{\pi}{2}\right\}$, o sistema (3.17) é singular. Porém, podemos considerar soluções saindo ortogonalmente de $\partial \Delta$ (vide Lema 0.6).

(ii) Soluções explícitas. Note que se $\mathbf{h} \equiv \pm(15 \cot a-7 \tan a)$, então $r=a$ é uma solução de (3.17) que gera uma esfera métrica de raio a com centro no ponto base $p$. Em particular a solução $r=\arctan \sqrt{\frac{15}{7}}$ gera uma esfera mínima, a saber a esfera métrica de volume máximo. Na outra mão $\theta=\frac{1}{2} \arctan \sqrt{\frac{5}{2}}$ é uma solução de (3.16) para $\mathbf{h} \equiv 0$ que gera uma hipersuperfície singular (no ponto base) homeomorfa à reunião (de espaços topológicos, identificando as fronteiras) de um cone sobre a órbita principal $\frac{S U(2) \times S U(4)}{U(1) \times S U(2)}$ e da aplicação cilíndrica do fibrado canônico (vide Observação 1.5(i))

$$
\mathbf{S}^{7} \hookrightarrow \frac{S U(2) \times S U(4)}{U(1) \times S U(2)} \longrightarrow \mathbf{S}^{2} \times \mathbf{S}^{5}
$$

Demonstração do Lema 3.23. Seja $H=\operatorname{Spin}(8)\left(\right.$ resp. $H=T^{1} \cdot \operatorname{Spin}(7)$ e $\left.S U(2) \cdot S U(4)\right)$ e consideremos a notação em §3.4.2. Sejam $\xi=\left[\begin{array}{l}0 \\ 1\end{array}\right], \eta=\left[\begin{array}{l}1 \\ 0\end{array}\right]\left(\operatorname{resp} . \eta=\left[\begin{array}{l}i \\ 0\end{array}\right]\right.$ e $\left.\left[\begin{array}{l}j \\ 0\end{array}\right]\right)$ em $\mathbf{O}^{2}=T_{p} \mathbf{P}_{\mathbf{O}}^{2}$ e $d=2$ (resp. $\left.d=4\right)$. Sejam $X, Y \in \mathfrak{M} \operatorname{com} X^{*} p=\xi$ e $Y_{p}^{*}=\eta$. Note que $Y \in \mathfrak{M}_{\lambda}$, logo $Z=[X, Y] \in \mathfrak{K}_{\lambda}$. Consideremos a geodésica $\gamma(r)=\operatorname{Exp}(r X)$. Para cada $\zeta \in \mathfrak{S O}(9)$ o campo de Jacobi $J_{\zeta}(r)$ ao longo de $\gamma(r)$, com $J_{\zeta}(0)=0$ e $J_{\zeta}^{\prime}(0)=[\zeta, X]$, é dado por

$$
J_{\zeta}(r)=\left\{\begin{array}{ll}
0, & \text { se } \zeta \in \mathfrak{K}_{0} \\
\sin r(\exp r X)_{*}[\zeta, X], & \text { se } \zeta \in \mathfrak{K}_{\lambda} . \\
\frac{\sin 2 r}{2}(\exp r X)_{*}[\zeta, X], & \text { se } \zeta \in \mathfrak{K}_{2 \lambda}
\end{array} .\right.
$$

Da Observação 3.24 e dos parágrafos $\S a)$ e $\S b)$ acima, temos que $F(r, \theta)=\exp \theta Z \cdot \gamma(r)$, $(r, \theta) \in\left[0 \frac{\pi}{2}\right] \times\left[0, \frac{\pi}{d}\right]$, é uma parametrização de $H \backslash \mathbf{P}_{\mathbf{O}}^{2}$. Fixemos $r \in\left(0, \frac{\pi}{2}\right)$ e consideremos a curva $\beta_{r}(\theta)=F(r, \theta)$. Note que as $H$-órbitas estão contidas nas esferas métricas, pois $H \subset \operatorname{Spin}(9)$. Temos que $\beta_{r}$ é uma geodésica em $S(r)$ que intersecta todas as $H$-órbitas ortogonalmente. Seja $t_{r}(\theta)$ o campo tangente unitário ao longo de $\beta_{r}(\theta)$. Assim $\frac{d}{d \theta} \beta_{r}=$ 
$\frac{\partial}{\partial \theta} F(r, \theta)=\left.(\exp \theta Z)_{*} \frac{d}{d \tau}\right|_{\tau=0} \exp \tau Z \cdot \gamma(r)$. Então de (3.18) tem-se

$$
t_{r}(\theta)=\frac{1}{\sin r} \frac{\partial F}{\partial \theta}=(\exp \theta Z)_{*}(\exp r X)_{*} Y
$$

Seja $N=N(r, \theta)$ a $H$-órbita de $\beta_{r}(\theta)$. Temos que $t_{r}(\theta)$ é normal a $N$ em $\beta_{r}(\theta)$. Na outra mão, o complemento ortogonal em $\mathfrak{H}$ da álgebra de Lie da isotropia principal coincide (nos três casos) com $\mathfrak{K}_{\lambda} \cap Z^{\perp}+\mathfrak{K}_{2 \lambda}$. Isto junto com (3.18) mostra que $T_{\beta_{r}(\theta)} N$ é gerado por $\left\{(\exp \theta Z)_{*} J_{\zeta}(r): \zeta \in \mathfrak{K}_{\lambda} \cap Z^{\perp}+\mathfrak{K}_{2 \lambda}\right\}$.

Seja $\psi(W)=\operatorname{Exp}(r W)$, para todo $W \in \mathfrak{M} \operatorname{com}|W|=1$. Se $\tilde{\beta}(\theta)=\operatorname{Ad}_{\exp (\theta Z)} X=$ $(\cos \theta) X+(\sin \theta) Y, \theta \in\left[0, \frac{\pi}{d}\right]$, então $\psi(\tilde{\beta})=\beta_{r}$. Agora fixemos $\theta \in\left(0, \frac{\pi}{d}\right)$. Dado $\zeta \in$ $\mathfrak{K}_{\lambda} \cap Z^{\perp}+\mathfrak{K}_{2 \lambda}$. De (3.18) segue que

$$
d \psi_{X}[\zeta, X]=\left.\frac{d}{d \tau}\right|_{\tau=0} \psi\left(\operatorname{Ad}_{\exp (\tau \zeta)} X\right)=\left.\frac{d}{d \tau}\right|_{\tau=0} \exp \tau \zeta \cdot \gamma(r)=J_{\zeta}(r) .
$$

Claramente $\psi$ é $H$-invariante. Portanto, se $\tilde{N}=\tilde{N}(\theta)$ é a $H$-órbita de $\tilde{\beta}(\theta)$, tem-se $T_{\tilde{\beta}(\theta)} \tilde{N}=$ $\mathfrak{M}_{\lambda} \cap Y+\mathfrak{M}_{2 \lambda}$. Sejam $d N$ e $d \tilde{N}$ as formas elemento de volume de $N$ e $\tilde{N}$, respectivamente. Então existe uma constante $\delta=\delta(r)$ tal que $\psi^{*} d N=\delta d \tilde{N}$. Para finalizar calculemos $\delta$. Seja $\left\{\zeta_{1}, \ldots \zeta_{14}\right\} \subset \mathfrak{K}_{\lambda} \cap Z^{\perp}+\mathfrak{K}_{2 \lambda}$ tal que $\left\{\left[\zeta_{1}, X\right], \ldots,\left[\zeta_{14}, X\right]\right\}$ é uma base ortonormal de $T_{\tilde{\beta}(\theta)} \tilde{N}$. Se $d S(r)$ é a forma elemento de volume de $S(r)$, então

$$
\begin{aligned}
\delta & =\delta d \tilde{N}(\tilde{\beta}(\theta))\left(\left[\zeta_{1}, X\right], \ldots,\left[\zeta_{14}, X\right]\right) \\
& =\psi^{*} d N(\tilde{\beta}(\theta))\left(\left[\zeta_{1}, X\right], \ldots,\left[\zeta_{14}, X\right]\right) \\
& =d N\left(\beta_{r}(\theta)\right)\left(\psi_{*}\left[\zeta_{1}, X\right], \ldots, \psi_{*}\left[\zeta_{14}, X\right]\right) \\
& =d N\left(\beta_{r}(\theta)\right)\left(J_{\zeta_{1}}(r), \ldots, J_{\zeta_{14}}(r)\right) \\
& =d S(r)\left(\beta_{r}(\theta)\right)\left(J_{\zeta_{1}}(r), \ldots, J_{\zeta_{14}}(r), t(\theta)\right) \\
& =2^{-7}(\sin r)^{7}(\sin 2 r)^{7} .
\end{aligned}
$$

\subsubsection{Análise da equação diferencial reduzida}

\begin{tabular}{|c|c|c|}
\hline & $T^{1} \cdot \operatorname{Spin}(7)$ & $S U(2) \cdot S U(4)$ \\
\hline$A$ & 6 & 5 \\
\hline$B$ & 1 & 2 \\
\hline
\end{tabular}

Tabela 3.1: Expoentes dependendo do grupo

Estudaremos os sistemas (3.16) e (3.17) para $\mathbf{h} \equiv 0$ de maneira simultânea. Com efeito, 
lembremos que a métrica orbital é

$$
d s^{2}=d r^{2}+\sin ^{2} r d \theta^{2}
$$

Na outra mão, o funcional de volume é dado por

$$
\mathscr{V}(r, \theta)=(\sin r)^{7}(\sin 2 r)^{7}(\sin 2 \theta)^{A}(\cos 2 \theta)^{B}
$$

onde $A$ e $B$ são como na Tabela 3.1. Logo esses sistemas (para $\mathbf{h} \equiv 0$ ) são da forma

$$
\begin{aligned}
& \frac{d r}{d s}=\cos \sigma \\
& \frac{d \theta}{d s}=\frac{\sin \sigma}{\sin r} \\
& \frac{d \sigma}{d s}=P(\theta) \frac{\cos \sigma}{\sin r}-Q(r) \sin \sigma
\end{aligned}
$$

com $P=\frac{\partial \ln \mathscr{V}}{\partial \theta}=2 A \cot 2 \theta-2 B \tan 2 \theta$ e $Q=\frac{\partial \ln \mathscr{V}}{\partial r}+\cot r=15 \cot r-7 \tan r$. Temos que $P$ e $Q$ decrescem monotonicamente e têm um único zero, a saber $\frac{1}{2} \arctan \sqrt{\frac{A}{B}}$ e $\arctan \sqrt{\frac{15}{7}}$, respectivamente. Também $\lim _{\theta \rightarrow 0^{+}} P(\theta)=+\infty=\lim _{r \rightarrow 0^{+}} Q(r)$ e $\lim _{\theta \rightarrow \frac{\pi}{4}^{-}} P(\theta)=-\infty=$ $\lim _{r \rightarrow \frac{\pi}{2}-} Q(r)$. Em diante usaremos a notação $R=\arctan \sqrt{\frac{15}{7}}$ e $T=\frac{1}{2} \arctan \sqrt{\frac{A}{B}}$.

Lema 3.31 (Pontos críticos locais). (i) Os pontos críticos locais de r são de máximo (resp. mínimo) se $r \in\left(R, \frac{\pi}{2}\right)$ (resp. $r \in(0, R)$ ).

(ii) Os pontos críticos locais de $\theta$ são de máximo (resp. mínimo) se $\theta \in\left(T, \frac{\pi}{4}\right)$ (resp. $\theta \in(0, T))$.

(iii) Os pontos críticos de $r$ (resp. $\theta$ ) são não degenerados exceto para a solução $r=R$ $($ resp. $\theta=T)$

(iv) Consideremos a função $f(r, \theta)=22 \sin ^{2} r+7 \tan ^{2} r-\frac{4 A}{(\sin 2 \theta)^{2}}-\frac{4 B}{(\cos 2 \theta)^{2}}$. Então, a curva $f(r, \theta)=0$ divide o interior de $\Delta$ em duas regiões conexas. Ainda mais, esta curva é da forma $r=\rho(\theta)$, sendo que $\rho$ tem um único ponto de máximo em $T_{0}=\frac{1}{2} \arctan \left(\frac{A}{B}\right)^{\frac{1}{4}} \in$ $\left(0, \frac{\pi}{4}\right)$, com $\rho\left(T_{0}\right)>R$. Em particular, a região $f>0$ está contida na região $r>R$. Ainda

$$
\lim _{\theta \rightarrow 0^{+}} \rho(\theta)=\frac{\pi}{2}=\lim _{\theta \rightarrow \frac{\pi}{4}^{-}} \rho(\theta)
$$

(v) Os pontos críticos de $\sigma$ são de máximo se $\sigma \in\left(-\frac{\pi}{2}, 0\right) \cup\left(\frac{\pi}{2}, \pi\right)$ e $f>0$ ou $\sigma \in$ $\left(-\pi,-\frac{\pi}{2}\right) \cup\left(0, \frac{\pi}{2}\right)$ e $f<0$. Os pontos críticos de $\sigma$ são de mínimo se $\sigma \in\left(-\pi,-\frac{\pi}{2}\right) \cup$ $\left(0, \frac{\pi}{2}\right)$ e $f>0$ ou $\sigma \in\left(-\frac{\pi}{2}, 0\right) \cup\left(\frac{\pi}{2}, \pi\right)$ e $f<0$. Em particular, os pontos críticos de $\sigma$ são não degenerados na região $f<0$ (resp. $f>0$ ), para soluções diferentes de $r=R$ $e \theta=T$. 


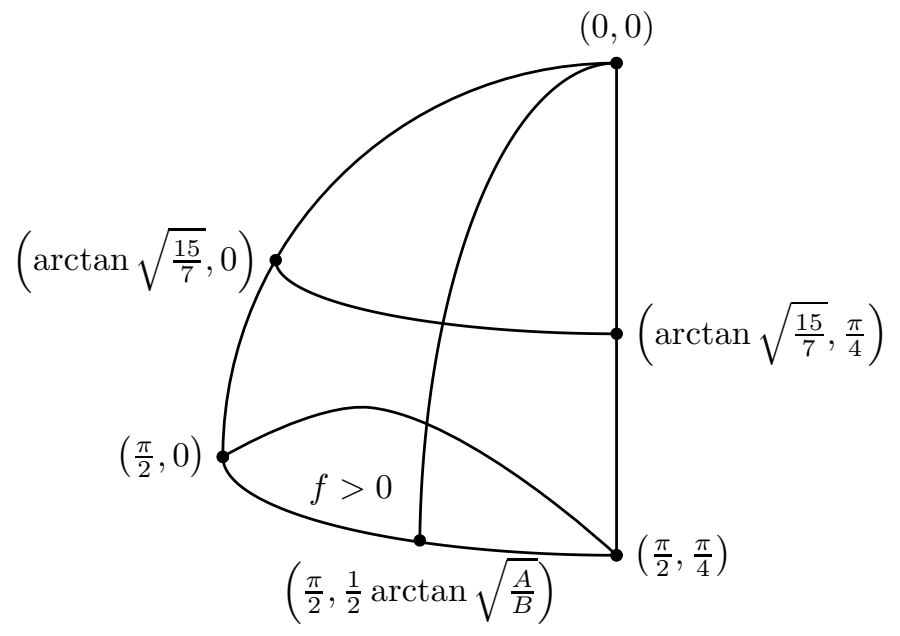

Figura 3.1: Regiões determinadas por $f(r, \theta)=0$

Demonstração. Das duas primeiras equações em (3.21) temos que se $\frac{d r}{d s}=0$ (resp. $\left.\frac{d \theta}{d s}=0\right)$, então $\frac{d^{2} r}{d s^{2}}=Q(r)$ (resp. $\frac{d^{2} \theta}{d s^{2}}=\frac{P(\theta)}{\sin ^{2} r}$ ). Assim obtemos (i) (resp. (ii)) e (iii). Para mostrar o item (iv) usamos o Teorema da Função Implícita. Com efeito $\frac{\partial f}{\partial r}>0$ e portanto $f^{-1}(0)$ é uma curva suave da forma $r=\rho(\theta), \theta \in\left(0, \frac{\pi}{4}\right)$, tal que $\frac{d \rho}{d \theta}=-\frac{\partial f}{\partial \theta} / \frac{\partial f}{\partial r}$. Na outra mão, $\frac{\partial f}{\partial \theta}=16 A \frac{\cos 2 \theta}{(\sin 2 \theta)^{3}}-16 B \frac{\sin 2 \theta}{(\cos 2 \theta)^{3}}$. Então, fazendo $T_{0}=\frac{1}{2} \arctan \left(\frac{A}{B}\right)^{\frac{1}{4}}$, temos que $\frac{\partial f}{\partial \theta}$ é positiva no intervalo $\left(0, T_{0}\right)$, anula-se em $T_{0}$ e é negativa no intervalo $\left(T_{0}, \frac{\pi}{4}\right)$. Logo $T_{0}$ é ponto de máximo de $\rho$. Seja $\rho_{0}=\rho\left(T_{0}\right)$, então $22 \sin ^{2} \rho_{0}+7 \tan ^{2} \rho_{0}=\frac{4 A}{\left(\sin 2 T_{0}\right)^{2}}+\frac{4 B}{\left(\cos 2 T_{0}\right)^{2}}=$ $4(\sqrt{A}+\sqrt{B})^{2}>30=22 \sin ^{2} R+7 \tan ^{2} R$. Portanto, como $\frac{\partial f}{\partial r}>0$, segue que $\rho_{0}>R$. Temos que $f(R, T)=-26$, assim $f>0$ implica $r>R$. Fazendo $\theta \rightarrow 0^{+}, \frac{\pi}{4}-$ na equação $f(r, \theta)=0$ obtemos $(3.22)$.

Finalmente diferenciando a terceira equação em (3.21) temos que

$$
\frac{d^{2} \sigma}{d s^{2}}=\frac{\sin 2 \sigma}{2 \sin ^{2} r} f(r, \theta)
$$

se $\frac{d \sigma}{d s}=0$. Assim obtemos a primeira afirmação de (v). Na outra mão, suponhamos que $\frac{\sigma}{d s}=0$ e $\sin \sigma=0$. Então, da terceira equação em (3.21) segue que $\theta=T$. Analogamente, se $\frac{\sigma}{d s}=0$ e $\cos \sigma=0$, então $r=R$.

Lembremos que, se $\gamma$ é geodésica dada em uma variedade riemanniana $M^{n}$, o campo de Jacobi $J$ ao longo de $\gamma$ satisfaz a equação de Jacobi seguinte:

$$
\frac{D^{2} J}{d t^{2}}+\mathscr{R}(V, J) V=0
$$

onde $V=\frac{d \gamma}{d t}$ é o vetor tangente unitário ao longo de $\gamma$ e $\mathscr{R}$ denota o tensor de Riemann de $M^{n}$. Se $E_{1}, \ldots, E_{n}$ é um referencial ortonormal e paralelo ao longo de $\gamma$ e fazemos $J(t)=\sum_{\alpha=1}^{n} f_{\alpha}(t) E_{\alpha}(t)$, então (3.23) transforma-se em

$$
\frac{d^{2} f_{\alpha}}{d t^{2}}+\sum_{\alpha=1}^{n} g_{\alpha, \beta}(t) f_{\alpha}(t), \quad \alpha=1, \ldots, n
$$


onde $g_{\alpha, \beta}=\left\langle\mathscr{R}\left(V, E_{\beta}\right) V, E_{\alpha}\right\rangle$. Em particular, no caso bidimensional, i.e. quando $n=2$, se $d s^{2}=E d x^{2}+F d y^{2}$ é a primeira forma fundamental de $M^{2}$, e $\gamma$ está parametrizada pelo comprimento de arco $s$; se $E_{1}=\frac{d \gamma}{d s}$ e $E_{2}$ é o vetor normal unitário, tal que $\left\{E_{1}, E_{2}\right\}$ está positivamente orientado. Então a equação de Jacobi ao longo de $\gamma$ é a seguinte equação diferencial ordinaria de segunda ordem:

$$
\frac{d^{2} f}{d s^{2}}+K(s) f(s)=0
$$

onde $f=f_{2}$ e $K$ é a curvatura gaussiana de $M^{2}$, a saber

$$
K=-\frac{1}{2 \sqrt{E G}}\left\{\frac{\partial}{\partial y} \frac{E_{y}}{\sqrt{E G}}+\frac{\partial}{\partial x} \frac{G_{x}}{\sqrt{E G}}\right\} .
$$

Como sabemos, as soluções de (3.21) são geodésicas em $\left(\Delta, d \bar{s}^{2}\right)$, onde $d \bar{s}^{2}=\mathscr{V}^{2} d s^{2}$ é a métrica modificada. Consideremos uma geodésica $\gamma$ em $\left(\Delta, d \bar{s}^{2}\right)$ parametrizada pelo comprimento de $\operatorname{arco} \bar{s}$. Então, de (3.19), (3.20) e (3.26) a curvatura gaussiana $K$ de $\Delta$ em relação a $d \bar{s}^{2}$ ao longo de $\gamma$ é da forma

$$
K(\bar{s})=\frac{1}{\mathscr{V}^{2} \sin ^{2} r}\left\{22 \sin ^{2} r+7 \tan ^{2} r+\frac{4 A}{(\sin 2 \theta)^{2}}+\frac{4 B}{(\cos 2 \theta)^{2}}\right\} .
$$

Em particular, ao longo das curvas $r=R$ e $\theta=T$ obtemos

$$
K(R, \theta)=\frac{1}{\mathscr{V}(R, \theta)^{2} \sin ^{2} R}\left\{30+\frac{4 A}{(\sin 2 \theta)^{2}}+\frac{4 B}{(\cos 2 \theta)^{2}}\right\}
$$

e

$$
K(r, T)=\frac{1}{\mathscr{V}(r, T)^{2}}\left\{22+\frac{56}{\sin ^{2} r}+\frac{7}{\cos ^{2} r}\right\}
$$

Logo, se $J_{R}(\bar{s})=x(\bar{s}) E_{2}^{R}(\bar{s})$ e $J_{T}(\bar{s})=y(\bar{s}) E_{2}^{T}(\bar{s})$, de (3.25) obtemos as equações de Jacobi ao longo de $r=R$ e $\theta=T$

$$
\frac{d^{2} x}{d \bar{s}^{2}}+\frac{1}{\mathscr{V}(R, \theta)^{2} \sin ^{2} R}\left\{30+\frac{4 A}{(\sin 2 \theta)^{2}}+\frac{4 B}{(\cos 2 \theta)^{2}}\right\} x=0
$$

e

$$
\frac{d^{2} y}{d \bar{s}^{2}}+\frac{1}{\mathscr{V}(r, T)^{2}}\left\{22+\frac{56}{\sin ^{2} r}+\frac{7}{\cos ^{2} r}\right\} y=0,
$$

respectivamente. Usando em cada caso as relações $\frac{d \bar{s}}{d \theta}=\mathscr{V}(R, \theta) \sin R$ e $\frac{d \bar{s}}{d r}=\mathscr{V}(r, T)$, para eliminar $\bar{s}$, obtemos

$$
\frac{d^{2} x}{d \theta^{2}}-(2 A \cot 2 \theta-2 B \tan 2 \theta) \frac{d x}{d \theta}+\left\{30+\frac{4 A}{(\sin 2 \theta)^{2}}+\frac{4 B}{(\cos 2 \theta)^{2}}\right\} x=0
$$

e

$$
\frac{d^{2} y}{d r^{2}}-(14 \cot r-7 \tan r) \frac{d y}{d r}+\left\{22+\frac{56}{\sin ^{2} r}+\frac{7}{\cos ^{2} r}\right\} y=0
$$


Por último, fazendo $u(\theta)=x(\theta)(\sin 2 \theta)^{\frac{A}{2}}(\cos 2 \theta)^{\frac{B}{2}}$ e $v(r)=x(r)(\sin r)^{7}(\cos r)^{\frac{7}{2}}$, temos que

$$
\frac{d^{2} u}{d \theta^{2}}+\left\{44+2 A B+\left(2 B-B^{2}\right) \tan ^{2} 2 \theta+\left(2 A-A^{2}\right) \cot ^{2} 2 \theta\right\} u=0
$$

e

$$
\frac{d^{2} v}{d r^{2}}+\left\{123.5-8.75 \tan ^{2} r\right\} v=0
$$

Lema 3.32. (i) Existem $\epsilon, \tau>0$ tais que toda solução de (3.34) definida em $\left[\tau, \frac{\pi}{4}-\epsilon\right]$ tem pelo menos um zero nesse intervalo.

(ii) Existe $\epsilon>0$ que toda solução $v=v(r)$ de (3.35) definida em $[\epsilon, R]$ tem pelo menos três zeros.

Demonstração. Note que as equações (3.34) e (3.35) são da forma

$$
\frac{d^{2} \xi}{d t^{2}}+q \xi=0
$$

Segue da Teoria de Sturm-Liouville (vide [Har02], Teorema 5.2, p.347) que se $N$ é o número de zeros de uma solução não trivial $\xi=\xi(t)$ da equação $(*)$ no intervalo $\left[t_{1}, t_{2}\right]$, com $q(t)>0$, para $t \in\left[t_{1}, t_{2}\right]$, então $\left|\pi N-\int_{t_{1}}^{t_{2}} \sqrt{q(t)} d t\right| \leqq \pi+\frac{1}{4} \int_{t_{1}}^{t_{2}} \frac{|d q(t)|}{q(t)}$. Em particular

$$
\pi(N+1) \geqq \int_{t_{1}}^{t_{2}} \sqrt{q(t)} d t-\frac{1}{4} \int_{t_{1}}^{t_{2}} \frac{|d q(t)|}{q(t)} .
$$

Usaremos a desigualdade $(* *)$ para estimar $N$ em cada caso.

Para mostrar o item (i), note que $q(\theta)=44+2 A B+\left(2 B-B^{2}\right) \tan ^{2} 2 \theta+\left(2 A-A^{2}\right) \cot ^{2} 2 \theta \geqq$ $p(\theta)=56-24 \cot ^{2} 2 \theta$, para todo $\theta \in\left(0, \frac{\pi}{4}\right)$. Então pelo Primeiro Teorema de Comparação de Sturm (vide [Har02], Teorema 3.1, p.334) é suficiente estimar o número de zeros de $p$. Sejam $\epsilon<10^{-4}$ e $\tau=\frac{1}{2} \arctan \sqrt{\frac{9}{50}}$. A função $p$ cresce monotonicamente e é positiva em $\left[\tau, \frac{\pi}{4}-\epsilon\right]$. Assim de $(* *)$ obtemos

$$
\begin{aligned}
\pi(N+1) & \geqq \int_{\tau}^{\frac{\pi}{4}-\epsilon} \sqrt{p(t)} d t+\frac{1}{4} \ln \left(\frac{p(\tau)}{p\left(\frac{\pi}{4}-\epsilon\right)}\right) \\
& >\int_{\tau}^{\frac{\pi}{4}-\epsilon} \sqrt{p(t)} d t+\frac{1}{4} \ln \left(\frac{p(\tau)}{p\left(\frac{\pi}{4}\right)}\right) \\
& \geqq\left(\frac{\pi}{4}-\epsilon-\tau\right) \sqrt{p(\tau)}+\frac{1}{4} \ln \left(\frac{p(\tau)}{p\left(\frac{\pi}{4}\right)}\right) \\
& =\left(\frac{\pi}{4}-\epsilon-\tau\right) \frac{\sqrt{1292}}{5}+\frac{1}{4} \ln \left(\frac{323}{350}\right) \\
& >(0.7853-0.0001-0.2006) \times(7.1888)-0.2008 \\
& =4.00177248 \\
& >\pi
\end{aligned}
$$


Logo $N \geqq 1$. Para mostrar o item (ii) seja $q(r)=123.5-8.75 \tan ^{2} r$. Temos que $q$ decresce monotonicamente. Seja $\epsilon=\arctan \sqrt{0.0004}$, assim $q>0$ em $[\epsilon, R]$. Logo de $(* *)$ temos que

$$
\begin{aligned}
\pi(N+1) & \geqq \int_{\epsilon}^{R} \sqrt{q(t)} d t+\frac{1}{4} \ln \left(\frac{q(R)}{q(\epsilon)}\right) \\
& \geqq(R-\epsilon) \sqrt{q(R)}+\frac{1}{4} \ln \left(\frac{q(R)}{q(\epsilon)}\right) \\
& =(R-\epsilon) \frac{\sqrt{409}}{2}+\frac{1}{4} \ln \left(\frac{409000}{493944}\right) \\
& >(0.9714-0.02) \times 10.1-0.0472 \\
& =9.56194 \\
& >3 \pi .
\end{aligned}
$$

Portanto $N \geqq 3$.

Definição 3.33. Para cada $a \in\left(0, \frac{\pi}{2}\right)$ seja $c_{a}(s)=\left(r_{a}(s), \theta_{a}(s), \sigma_{a}(s)\right)$ a solução de $(3.21)$ com valores iniciais $c_{a}(0)=\left(a, 0, \frac{\pi}{2}\right)$. Seja $\gamma_{a}(s)=\left(r_{a}(s), \theta_{a}(s)\right)$ a projeção de $c_{a}$ em $\Delta$.

Definição 3.34. Sejam $I, I I, I I I$ e $I V$ as seguintes regiões abertas de $\Delta$ :

$$
\begin{array}{cl}
I: & (0, R) \times(0, T), \\
I I: & (0, R) \times\left(T, \frac{\pi}{4}\right), \\
I I I: & \left(R, \frac{\pi}{2}\right) \times\left(T, \frac{\pi}{4}\right), \\
I V: & \left(R, \frac{\pi}{2}\right) \times(0, T) .
\end{array}
$$

Seja $\Delta^{-}$o conjunto dos pontos no interior de $\Delta$ onde $f<0$. Note que, $I, I I \subset \Delta^{-}$. Sejam $I I I^{-}$e $I V^{-}$as intersecções de $\Delta^{-}$com $I I I$ e $I V$, respectivamente.

Estudaremos a família a um parâmetro de soluções de (3.21) na Definição 3.33, para $a \in(0, R)$.

Lema 3.35. Seja $a \in(0, R)$, então

(i) $\frac{d \sigma_{a}}{d s}(0)=-\frac{Q(a)}{A+1}<0$.

(ii) $\gamma_{a}$ passa de I para II cruzando $\theta=T \operatorname{com} r_{a}<R$.

Demonstração. O item (i) segue de multiplicar a terceira equação em (3.21) por sin $4 \theta$, diferenciar e computar em $s=0$. Agora, do Lema 3.31(v), segue que $\sigma_{a}$ não atinge mínimo em $\Delta^{-}$enquanto permanece em $\left(0, \frac{\pi}{2}\right)$. Pelo item (ii) do mesmo Lema $\theta_{a}$ não atinge máximo em $I$. Então, $\sigma_{a} \in\left(0, \frac{\pi}{2}\right)$ enquanto $\gamma_{a}$ está em $I$. Segue que $\gamma_{a}$ deve sair de $I$ em algum instante. A saída deve acontecer através de $\theta=T, 0<r<R$, pois pela terceira equação em 3.21, ao longo de $r=R$ temos $\frac{d \sigma}{d s}=P(\theta) \frac{\cos \sigma}{\sin r} \geq 0$ e $\frac{d \sigma}{d s}=0$ ocorre apenas se $\theta=T$. Mas, $\sigma_{a}$ não tem mínimo em $\Delta^{-}$. Isto mostra o item (ii). 
Definição 3.36. Para cada $a \in\left(0, \frac{\pi}{2}\right)$ sejam $r_{a, M}^{i}$ (resp. $r_{a, m}^{i}$ ) o $i$-ésimo máximo (resp. mínimo) local de $r_{a}$ e $\theta_{a, M}^{i}$ (resp. $\theta_{a, m}^{i}$ ) o $i$-ésimo máximo (resp. mínimo) local de $\theta_{a}$. O padrão de pontos críticos locais $\mathbb{P}\left(\gamma_{a}\right)$ de $\gamma_{a}$ é a seqüência ordenada dos respectivos símbolos acima para $\gamma_{a}$.

Observação 3.37. Note que pode acontecer o caso de $r_{a}$ e $\theta_{a}$ terem um ponto crítico para o mesmo valor de $s$, digamos $s^{\prime}$. De fato isso sucede se e somente se $\gamma_{a}\left(s^{\prime}\right) \in \partial \Delta$. Neste caso os correspondentes símbolos serão denotados dentro de um colchete separados por uma vírgula.

Lema 3.38 (Vide [HT87] p. 208). Segmentos compactos de soluções de (3.21) dependem continuamente das condições iniciais, inclusive se contêm pontos em $\partial \Delta$.

Embora a equação diferencial reduzida em [HT87] seja outra, a prova do Lema 3.38 pode ser adaptada ao nosso caso.

Proposição 3.39. Consideremos $a \in(0, R)$. Existe $\delta>0$ tal que se $R-a<\delta$, então $\mathbb{P}\left(\gamma_{a}\right)=\left\{\theta_{a, M}^{1}, r_{a, M}^{1}, \ldots\right\}$.

Demonstração. Da demonstração do Lema 3.35 segue que $\sigma_{a} \in\left(0, \frac{\pi}{2}\right)$ em quanto $\gamma_{a}$ permanece em $I$. Logo $r_{a}$ decresce em $I$. Sejam $\epsilon$ e $\tau$ como no Lema 3.32(i). Então, para $\delta>0$ suficientemente pequeno, os segmentos das curvas $\gamma_{a}$ a partir de $\theta=\tau$, para $a \in(R-\delta, R)$ constituem uma variação por geodésicas (na métrica modificada) de $r=R$. Então, pelo Lema 3.32(i) o campo de Jacobi associado a tal variação se anula pelo menos uma vez no intervalo $\left(\tau, \frac{\pi}{4}-\epsilon\right)$. Assim, diminuindo $\delta$ se necessário, temos que $\gamma_{a}$ cruza $r=R$ pelo menos uma vez antes $\theta_{a, M}^{1}$, para todo $a \in(R-\delta, R)$. Pelo Lema 3.35(ii) tal cruzamento acontece para $\gamma_{a}$ passando de $I I$ para $I I I$. Pelo Lema 3.38 temos que, diminuindo de novo $\delta, \gamma_{a}$ fica próximo de $r=R$ pelo menos até cruzar $\theta=\frac{\pi}{4}-\epsilon$, com $\sigma_{a} \in\left(0, \frac{\pi}{2}\right)$. Em particular, $\gamma_{a}$ fica em $I I I^{-}$com $\sigma_{a} \in\left(0, \frac{\pi}{2}\right)$. Logo, como $\sigma_{a}$ não tem mínimo nessa região vide Lema 3.31(iv), $\sigma_{a}=0$ em algum instante ou $\gamma_{a}$ chega em $\theta=\frac{\pi}{4}$. A segunda opção é impossível pelo Lemma 0.6. Enfim, usando de novo o Lema 3.35(i), mostramos que $\gamma_{a}$ cruza novamente $r=R$ pelo menos uma vez antes de $\theta_{a, m}^{1}$. Assim $\sigma_{a}=\frac{\pi}{2}$ e $r_{a}=r_{a, M}^{1}$ em algum instante antes de $\gamma_{a}$ cruzar pela segunda vez $r=R$.

Seja $\mathscr{U}=\mathbf{P}_{\mathbf{O}}^{2}-\operatorname{Cut}(p)$ a vizinhaça normal de raio maximal do ponto base $p \in \mathbf{P}_{\mathbf{O}}^{2}$. Consideremos a família a um parâmetro de homotetias $\phi_{\lambda}, \lambda \in(0,1)$, dadas por

$$
\phi_{\lambda}(x)=\operatorname{Exp}\left(\lambda \operatorname{Exp}^{-1}(x)\right)
$$

para todo $x \in \mathscr{U}$. Seja $g$ a métrica riemanniana de $\mathbf{P}_{\mathbf{O}}^{2}$ e consideremos as métricas $g_{\lambda}=$ $\frac{1}{\lambda^{2}} \phi_{\lambda}^{*} g$ definidas em $\mathscr{U}$. Denotemos com $\mathscr{U}_{\lambda}$ a variedade riemanniana $\left(\mathscr{U}, g_{\lambda}\right)$. Temos que $g_{\lambda}$ é $\operatorname{Spin}(9)$ invariante, pois $\phi_{\lambda}$ leva esferas métricas em esferas métricas (de radio menor). 
Segue que $H=T^{1} \cdot \operatorname{Spin}(7)$ (resp. $\left.S U(2) \cdot S U(4)\right)$ atua por isometrias sobre $\mathscr{U}_{\lambda}$ e que o par $\left(H, \mathscr{U}_{\lambda}\right)$ é polar. Na outra mão, se $\mathscr{V}_{\lambda}$ denota o funcional de volume de $\left(H, \mathscr{U}_{\lambda}\right)$ temos que $\mathscr{V}_{\lambda}(x)=\frac{1}{\lambda^{14}} \mathscr{V}\left(\phi_{\lambda}(x)\right)$, para todo $x \in \mathscr{U}$. O espaço das $H$-órbitas $H \backslash \mathscr{U}_{\lambda}$ é $\Delta_{\lambda}=\{(r, \theta)$ : $0 \leq r \leq \frac{\pi}{2 \lambda}$ e $\left.0 \leq \theta \leq \frac{\pi}{4}\right\}$. A métrica orbital e o funcional de volume respectivos são dados por

$$
d s_{\lambda}^{2}=d r^{2}+\left(\frac{\sin \lambda r}{\lambda}\right)^{2} d \theta^{2} \text { e } \mathscr{V}_{\lambda}(r, \theta)=\frac{1}{\lambda^{14}} \mathscr{V}(\lambda r, \theta)
$$

Assim obtemos a equação diferencial reduzida (compare com (3.21))

$$
\begin{aligned}
\frac{d r}{d s_{\lambda}} & =\cos \sigma \\
\frac{d \theta}{d s_{\lambda}} & =\frac{\lambda \sin \sigma}{\sin \lambda r} \\
\frac{d \sigma}{d s_{\lambda}} & =P(\theta) \frac{\lambda \cos \sigma}{\sin \lambda r}-\lambda Q(\lambda r) \sin \sigma
\end{aligned}
$$

onde $P$ e $Q$ são as mesmas funções envolvidas em (3.21). De fato, pode-se mostrar que se $\lambda \rightarrow 0$ então $\mathscr{U}_{0}=T_{p} \mathbf{P}_{\mathbf{O}}^{2}$ com a métrica $g_{0}=g(p)$. Isto é, os coeficientes da forma quadrática dada por $g_{\lambda}$ (e suas derivadas de qualquer ordem) convergem uniformemente aos respectivos coeficientes de $g_{0}$. Neste caso $\left(H, \mathscr{U}_{0}\right)$ é a ação slice. Logo $H \backslash \mathscr{U}_{0}=\Delta_{0}=\{(r, \theta)$ : $r \geq 0$ e $\left.0 \leq \theta \leq \frac{\pi}{4}\right\}$. A métrica orbital e o funcional de volume respectivos são dados por

$$
d s^{2}=d r^{2}+r^{2} d \theta^{2} \text { e } \mathscr{V}_{0}(r, \theta)=r^{14}(\sin 2 \theta)^{A}(\cos 2 \theta)^{B}
$$

A equação diferencial reduzida limite é

$$
\begin{aligned}
& \frac{d r}{d s}=\cos \sigma \\
& \frac{d \theta}{d s}=\frac{\sin \sigma}{r} . \\
& \frac{d \sigma}{d s}=P(\theta) \frac{\cos \sigma}{r}-\frac{15 \sin \sigma}{r}
\end{aligned}
$$

Observação 3.40. (i) Note que as homotetias $\phi_{\lambda}: \mathscr{U}_{\lambda} \rightarrow \mathscr{U}_{1}$, são $H$-equivariantes e transformam hipersuperfícies mínimas em hipersuperfícies mínimas. As homotetias induzidas $\Delta_{\lambda} \rightarrow \Delta_{1}$ são dadas por $(r, \theta) \mapsto(\lambda r, \theta)$. Portanto, estas levam soluções de (3.36) em soluções de (3.21).

(ii) Para todo $\lambda \in[0,1]$ o sistema (3.36) é singular no bordo $\partial \Delta_{\lambda}$. No entanto, podemos considerar soluções saindo ortogonalmente de $\partial \Delta_{\lambda}$ (vide Lema 0.6).

(iii) Soluções de (3.36) dependem analiticamente de $\lambda$, pois as funções $\cos \lambda r$ e $\frac{\lambda}{\sin \lambda r}$ são anaíticas em $(\lambda, r)$ inclusive para $\lambda=0$. 
Proposição 3.41. (i) Dados $\epsilon>0$ e $0<\tau<T$, existe $\delta>0$ tal que se $a \in(0, \delta)$ então $\gamma_{a}$ cruza $r=\epsilon \operatorname{com} \tau<\theta_{a}<T$.

(ii) Existe $\delta>0$ tal que se $a \in(0, \delta)$ então $\mathbb{P}\left(\gamma_{a}\right)=\left\{\theta_{a, M}^{1}, \theta_{a, m}^{1}, \ldots, r_{a, M}^{1}, \ldots\right\}$.

Demonstração. Para cada $\lambda \in[0,1]$ seja $c_{\lambda, a}(s)=\left(r_{\lambda, a}(s), \theta_{\lambda, a}(s), \sigma_{\lambda, a}(s)\right)$ a solução de (3.36) com condições iniciais $c_{\lambda, a}(0)=\left(a, 0, \frac{\pi}{2}\right)$. Seja $\gamma_{\lambda, a}$ a projeção de $c_{\lambda, a}$ em $\Delta_{\lambda}$. Pela demostração do Lema 3.35(i) temos que $\frac{d \sigma_{\lambda, a}}{d s_{\lambda}}(0)<0$. Consideremos primeiramente $\lambda=0$. Se verifica facilmente de (3.37) que, se $\theta_{0, a} \in(0, T)$, então $\sigma_{0, a} \in\left(0 \frac{\pi}{2}\right)$. Portanto, na região $0<\theta<T, \gamma_{0, a}$ é da forma $r=r(\theta)$. Neste caso, podemos considerar o seguinte sistema equivalente a (3.37)

$$
\begin{aligned}
\frac{1}{r} \frac{d r}{d \theta} & =\cot \sigma \\
\frac{d \sigma}{d \theta} & =P(\theta) \cot \sigma-15 .
\end{aligned}
$$

Seja $\varphi$ o ângulo entre o vetor tangente unitário a $\gamma$ e $\frac{\partial}{\partial x}$ (coordenadas cartesianas $\left.(x, y)\right)$. Então $\varphi=\sigma+\theta$. Assim (3.38) se transforma em

$$
\frac{d \varphi}{d \theta}=P(\theta) \cot (\varphi-\theta)-14
$$

Multiplicando a equação (3.39) por $\sin 4 \theta \sin (\varphi-\theta)$ obtemos

$$
\sin 4 \theta \sin (\varphi-\theta) \frac{d \varphi}{d \theta}=28 \cos 2 \theta \cos (\varphi+\theta)-4 B \cos (\varphi-\theta)
$$

ou, equivalentemente, o sistema planar não linear

$$
\begin{aligned}
& \frac{d \theta}{d t}=\sin 4 \theta \sin (\varphi-\theta) \\
& \frac{d \varphi}{d t}=28 \cos 2 \theta \cos (\varphi+\theta)-4 B \cos (\varphi-\theta)
\end{aligned}
$$

Pode-se verificar facilmente que $\left(0, \frac{\pi}{2}\right)$ e $(T, T)$ são pontos singulares isolados de (3.40) do tipo sela e nodal estável, respectivamente. Ainda mais estes são os únicos pontos singulares de (3.40) em $\mathscr{R}=[0, T] \times\left[T, \frac{\pi}{2}\right]$. A solução de (3.39) com $\varphi(0)=\frac{\pi}{2}$ é precisamente uma curva integral de (3.40), contida em $\mathscr{R}$, com $\lim _{t \rightarrow 0^{+}} \varphi(t)=\frac{\pi}{2}$ e $\lim _{t \rightarrow T^{-}} \varphi(t)=T$. Segue que, $\theta_{0, a}$ é assintótica à reta $\theta=T$ e não a cruza. Então $\sigma_{0, a} \in\left(0, \frac{\pi}{2}\right)$ e portanto $r_{0, a}$ e $\theta_{0, a}$ são funções crescentes. Ainda mais, da primeira equação em (3.38) tem-se que, se $r_{0, a}=r(\theta)$ e $\sigma_{0, a}=\sigma(\theta)$, então $r(\theta)=a \mathrm{e}^{\int_{0}^{\theta} \cot (\sigma(t)) d t}$.

Sejam $\epsilon>0$ e $\tau \in(0, T)$. Então existe $\rho_{0}>1$ tal que $\gamma_{0,1}$ cruza $r=\rho_{0} \operatorname{com} \theta_{0,1}=\tau$. Assim, para $a \in(0,1) \gamma_{0, a}$ cruza $r=\rho_{0} \operatorname{com} \tau<\theta_{0, a}<T$. Pela Observação 3.40(iii) existe 


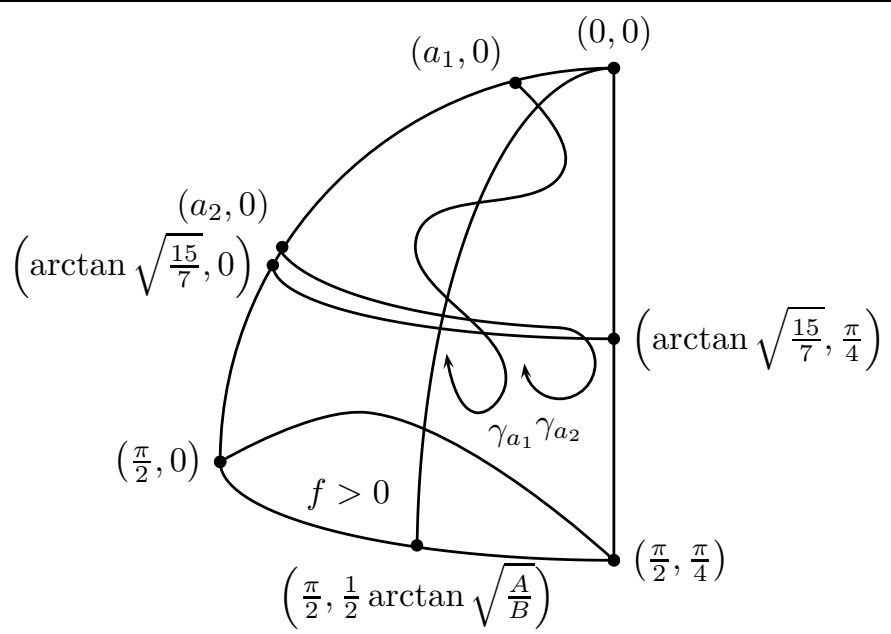

Figura 3.2: Padrão de pontos críticos da curva $\gamma_{a}$, para $a$ perto de 0 (resp. perto de $\left.\arctan \sqrt{\frac{15}{7}}\right)$

$\lambda \in(0,1)$ suficientemente pequeno tal que $\frac{\pi}{2 \lambda}>\rho_{0}$ e $\gamma_{\lambda, a}$ aproxima $\gamma_{0, a}, a \in(0,1)$, i.e. $\gamma_{\lambda, a}$ cruza $r=\rho_{0} \operatorname{com} \theta_{\lambda, a} \in(\tau, T)$. O item (i) segue da Observação 3.40(i) usando a homotetia $(r, \theta) \mapsto(\lambda r, \theta)$ e diminuindo $\lambda$ se necessário para obter $\epsilon=\lambda \rho_{0}$. Para mostrar o item (ii), consideremos $0<\tau<T$. Seja $\epsilon$ como no Lema 3.32(ii) e $\delta=\delta(\epsilon, \tau)$ como no item (i). Então, para $\tau$ suficientemente próximo a $T$, obtemos uma variação por geodésicas (na métrica modificada) da curva $\theta=T$. Pelo Lema 3.32(ii) o campo de Jacobi correspondente anula-se pelo menos três vezes em $[\epsilon, R]$. Logo, diminuindo $\delta$, se necessário, temos que $\gamma_{a}$ cruza $\theta=T$ pelo menos três vezes antes de $r_{a, M}^{1}$ para todo $a \in(0, \delta)$.

Corolário 3.42. Existe $a_{0} \in\left(0, \arctan \sqrt{\frac{15}{7}}\right)$ tal que $\mathbb{P}\left(\gamma_{a_{0}}\right)=\left\{\theta_{a_{0}, M},\left[r_{a_{0}, M}^{i}, \theta_{a_{0}, m}^{i}\right], \ldots\right\}$. Portanto $\mathbb{P}\left(\gamma_{a_{0}}\right)$ é periódico com período

$$
\theta_{a_{0}, M}^{1},\left[r_{a_{0}, M}^{1}, \theta_{a_{0}, m}^{1}\right], \theta_{a_{0}, M}^{2},\left[r_{a_{0}, m}^{1}, \theta_{a_{0}, m}^{2}\right]
$$

Portanto $\gamma_{a_{0}}$ sai ortogonalmente da aresta $\theta=0$ e chega ortogonalmente na aresta $r=\frac{\pi}{2}$ sem auto interseções.

Demonstração. Pelo Lema 3.31, os pontos críticos de $r_{a}$ e $\theta_{a}$ são não degenerados. Segue que $r_{a, M}^{i}, r_{a, m}^{i}, \theta_{a, M}^{i}$ e $\theta_{a, m}^{i}$ dependem continuamente de $a \in(0, R)$. Assim o resultado segue, por um argumento de continuidade, das proposições 3.39 e 3.41(ii).

Observação 3.43. Note que estudando a família $c_{a}(s)=\left(r_{a}(s), \theta_{a}(s), \sigma_{a}(s)\right), a \in(0, R)$, de soluções de $(3.21)$ com valores iniciais $c_{a}(0)=\left(a, \frac{\pi}{4},-\frac{\pi}{2}\right)$, podemos construir uma solução tal que sua projeção em $\Delta$ saia ortogonalmente da aresta $\theta=\frac{\pi}{4}$ e chegue ortogonalmente na aresta $r=\frac{\pi}{2}$ sem auto interseções. Para isto usamos resultados análogos ao Lema 3.35, às proposições 3.39, 3.41 e ao Corolário 3.42 .

Lema 3.44. Sejam $m \geq 3$ e $\ell$ uma reta em $\mathbf{R}^{m}$. Seja $T^{1} \rightarrow S O(m)$ representado pelas rotações em torno de $\ell$. Então o caminho c em $S O(m)$ descrito por $T^{1}$ é homotópico ao caminho inverso. 
Demonstração. Com efeito, vejamos que $[c]=[-c]$. Consideremos um 2-plano $E$ contendo $\ell$. A rotação continua de 180 graus de $\ell$ ao longo de $E$ define uma homotopia entre $c$ e $-c$.

Demonstração do Teorema 0.5. Seja $H=T^{1} \cdot \operatorname{Spin}(7)$ (resp. $\left.S U(2) \cdot S U(4)\right)$. Note que o espaço das $H$-órbitas $\left(\Delta, d s^{2}\right)$ é um triângulo geodésico na esfera $\mathbf{S}^{2}$, parametrizado em coordenadas polares $(r, \theta)$. Consideremos as soluções de (3.21) dadas pelo Corolário 3.42 e a Observação 3.43. As curvas em $\Delta$ correspondentes são difeomorfas a arcos de circunferência com raio suficientemente pequeno e centros nos vértices $\left(\frac{\pi}{2}, 0\right)$ e $\left(\frac{\pi}{2}, \frac{\pi}{4}\right)$, respectivamente, (vide Figura 3.3). Sejam $q$ e $q^{\prime}$ os pontos em $M$ correspondentes a $\left(\frac{\pi}{2}, 0\right)$ e $\left(\frac{\pi}{2}, \frac{\pi}{4}\right)$. Temos que

$$
q=\left[\begin{array}{lll}
0 & 0 & 0 \\
0 & 1 & 0 \\
0 & 0 & 0
\end{array}\right] \text { e } q^{\prime}=\left[\begin{array}{ccc}
\frac{1}{2} & \frac{i}{2} & 0 \\
-\frac{i}{2} & \frac{1}{2} & 0 \\
0 & 0 & 0
\end{array}\right] \quad\left(\text { resp. } q^{\prime}=\left[\begin{array}{ccc}
\frac{1}{2} & \frac{j}{2} & 0 \\
-\frac{j}{2} & \frac{1}{2} & 0 \\
0 & 0 & 0
\end{array}\right]\right)
$$

Cada um destes arcos gera uma hipersuperfície difeomorfa à fronteira de um tubo de raio constante sobre a órbita $H(q) \approx \mathbf{S}^{1}$ (resp. $\left.\mathbf{S}^{2}\right)$ e $H\left(q^{\prime}\right) \approx \mathbf{S}^{6}$ (resp. $\left.\mathbf{S}^{5}\right)$, respectivamente. Estas por sua vez são difeomorfas aos fibrados (vide Proposição 1.6)

$$
S \hookrightarrow H \times_{H_{x}} S \longrightarrow H(x)
$$

onde $S$ é a esfera unitária em $\nu_{x} H(x)$, para $x=q, q^{\prime}$; i.e. $S=\mathbf{S}^{14}, \mathbf{S}^{9}$ (resp. $\mathbf{S}^{13}, \mathbf{S}^{10}$ ). De [Ste99] $§ 26$ segue que há somente dois tipos de fibrados de $m$-esferas sobre $\mathbf{S}^{1}$, para $m>1$, a saber orientáveis (triviais) e não orientáveis (garrafas de Klein generalizadas). Segue também que fibrados de 9-esferas (resp. 10-esferas) sobre $\mathbf{S}^{6}$ (resp. $\mathbf{S}^{5}$ ) são triviais. Para finalizar a prova vejamos que os fibrados (3.41) para $x=q$ são triviais.

(i) Seja $H=T^{1} \times \operatorname{Spin}(7)$. Neste caso $\nu_{p} H(q)=\left\{\left[\begin{array}{l}u \\ v\end{array}\right] \in \mathbf{O}^{2}: u \in \Im(\mathbf{O})\right\}$ e $H_{q}=$ $T^{1} \times S U(4)$. A representação slice $\left(H_{q}, \nu_{q} H(q)\right)$ é dada por

$$
\rho( \pm \mathrm{id}, g)\left[\begin{array}{l}
u \\
v
\end{array}\right]=\left[\begin{array}{c}
g_{1} u \\
\pm g_{2} v
\end{array}\right]
$$

Portanto $\rho\left(H_{q}\right) \subset S O(15)$. Portanto (3.41) para $x=q$ é orientável.

(ii) Seja $H=S U(2) \times S(4)$. Neste caso $\nu_{p} H(q)=\left\{\left[\begin{array}{l}u \\ v\end{array}\right] \in \mathbf{O}^{2}: u \in \mathbf{C}^{\perp} \subset \mathbf{O}\right\}$ e $H_{q}=$ $T^{1} \times S U(4)$. A representação slice $\left(H_{q}, \nu_{q} H(q)\right)$ é dada por

$$
\rho(\lambda, g)\left[\begin{array}{l}
u \\
v
\end{array}\right]=\left[\begin{array}{c}
g_{1} u \\
\lambda g_{2} v
\end{array}\right]
$$

Temos $H_{q}$ é conexo, logo $\rho\left(H_{q}\right) \subset S O(15)$. Sejam $h_{n}$ e $h_{s}$ os hemisférios (fechados) norte e sul de $H(q)=\mathbf{S}^{2}$. Sejam $\mathscr{U}_{n}$ e $\mathscr{U}_{s}$ dois abertos de $\mathbf{S}^{2}$ contendo $h_{n}$ e $h_{s}$, respectivamente, 


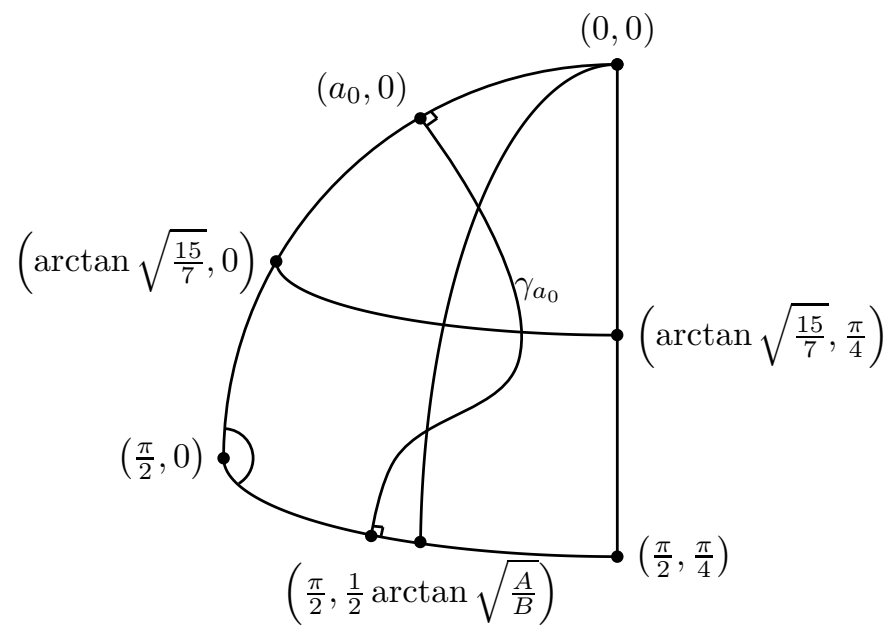

Figura 3.3: Soluções de (3.17) saindo (resp. chegando) ortogonalmente de $\theta=0$ (resp. em $\left.r=\frac{\pi}{2}\right)$

tais que $\mathscr{U}_{n} \cap \mathscr{U}_{s}$ é uma anel cuja fronteira e a reunião de dois círculos paralelos ao equador $h_{n} \cap h_{s}=\mathbf{S}^{\mathbf{1}}$. Sejam $t_{n}$ e $t_{s}$ duas trivializações de $(3.41)(x=q)$ em $\mathscr{U}_{n}$ e $\mathscr{U}_{s}$, respectivamente. Consideremos o caminho fechado $c: \mathbf{S}^{1} \rightarrow \rho\left(H_{q}\right) \subset S O(15)$, dado pela restrição de $\left.t_{n} t_{s}^{-1}\right|_{\mathbf{S}^{1}}$. O resultado segue de [Ste99] $\S 18.3$ se mostrarmos que a classe de homotopia de $c$ em $\pi_{1}(S O(15))=\mathbf{Z}_{2}$ é trivial. Isto segue sem dificuldade, usando a idéia na demonstração do Lema 3.44, para ver que $c$ é homotópico em $S O(15)$ a um caminho em $S U(4)$. 


\section{Referências bibliográficas}

[Ada96] J.F. Adams. Lecture notes on exceptional lie groups. Chicago Lectures in Mathematics, chapter 15. The University of Chicago Press, Chicago-London, 1996.

[AK07] B. Apanasov and I. Kim. Cartan angular invariant and deformations in rank one symetric spaces. Sb. Math., 198(1-2):147-169, 2007.

[Ale11] M.M. Alexandrino. On polar foliations and the fundamental group. Results Math., 60(1-4):213-223, 2011.

[Arv03] A. Arvanitoyeorgos. An introduction to lie groups and the geomety of homogeneous spaces. volume 22 of Student Mathematical Library. American Mathematical Society, 2003.

[BCO03] J. Berndt, S. Console, and C. Olmos. Submanifolds and holonomy. volume 434 of Research Notes in Mathematics. Chapman \& Hall/CRC, Boca Raton-London-New York-Washington, D.C., 2003.

[BGS85] W. Ballmann, M. Gromov, and V. Schoeder. Manifolds of nonpositive curvature. volume 61 of Progress in Mathematics. Birkhäuser, Boston-Basel-Stutgart, 1985.

[CR98] L.M. Chaves and A. Rigas. From the triality viewpoint. Note Math., 18(2):155163, 1998.

[CS03] J.H. Conway and D.A. Smith. On quaternions and octonions, chapter 6. A K Peters, first edition, 2003.

[dCD83] M. do Carmo and M. Dajczer. Rotation hypersurfeces in spaces of constant curvature. Transactions of the American Mathematical Society, 277:685-709, 1983.

[Dui00] J.J. Duistermaat. Lie groups. Universitext, chapter 2. Springer, Berlin-HeidelbergNew york-Barcelona-Hong Kong-London-Milan-Paris-Singapore-Tokyo, 2000. 
[Fre85] H. Freudenthal. Oktaven, ausnahmegruppen und oktavengeometrie. Geometriae Dedicata, 19:7-63, 1985.

[GG00] C. Gorodski and N. Gusevskii. Complete minimal hypersurfaces in complex hyperbolic spaces. Manuscripta Math., 103:221-240, 2000.

[Gol06] M.W. Goldman. Complex hyperbolic geometry. Oxford Mathematical Monographs. Oxford University Press, 2006.

[Gor94] C. Gorodski. Minimal sphere bundles in euclidean spheres. Geometriae Dedicata, (53):75-102, 1994.

[Gor96] C. Gorodski. Minimal hyperspheres in rank two compact symmetric spaces. Bol. Soc. Bras. Mat., 27(1):1-22, 1996.

[Gor10] C. Gorodski. Symmetric spaces, chapter 5. 2010. Notas de curso.

[GP92] W.M. Goldman and J.R. Parker. Dirichlet polyhedra for dihedral groups acting on complex hyperbolic space. The Journal of Geometric Analysis, 2(6):517-553, 1992.

[Har02] P. Hartman. Ordinary differential equations. volume 38 of Classics in Applied Mathematics, chapter XI. Society for Industrial ans Applied Mathematics, Philadelphia, 2002.

[Hel78] S. Helgason. Differential geometry, lie groups and symmetric spaces. volume 80 of Pure and Applied Mathematics, chapter VI. Academic Press, San Diego-New York-Boston-London-Sydney-Tokyo-Toronto, 1978.

[HH80] W.-T. Hsiang and W.-Y. Hsiang. Examples of codimensional-one closed minimal submanifolds in some symmetric spaces. I. J. Differential Geom., 15:543-551, 1980.

[HH82] W.-T. Hsiang and W.-Y. Hsiang. On the existence of codimension-one minimal spheres in compact symmetric spaces of rank 2. II. J. Differential Geom., 17:583$594,1982$.

[HHT88] W.-T. Hsiang, W.-Y. Hsiang, and P. Tomter. On the existence of minimal hyperspheres in compact symmetric spaces. Ann. scient. Éc. Norm. Sup., 21:287$305,1988$.

[HL71] W.-Y. Hsiang and H.B. Jr Lawson. Minimal submanifolds of low cohomogeneity. J. Differential Geom., 5:1-38, 1971.

[HS86] W.-Y. Hsiang and I. Sterling. Minimal cones and the spherical Bernstein problem. III. Invent. Math., 85:223-247, 1986. 
[Hsi83a] W.-Y. Hsiang. Minimal cones and the spherical Bernstein problem I. Annals of Mathematics, 118:61-73, 1983.

[Hsi83b] W.-Y. Hsiang. Minimal cones and the spherical Bernstein problem. II. Invent. Math., 74:351-369, 1983.

[HT87] W.-Y. Hsiang and P. Tomter. On minimal immersions of $S^{n-1}$ into $S^{n}(1), n \geq 4$. Ann. scient. Éc. Norm. Sup., 20:201-214, 1987.

[Iwa81] K. Iwata. Compact transformation groups on rotational cohomology cayley projective planes. Tôhoku Math. Journ., 33:429-442, 1981.

[Kna96] A.W. Knapp. Lie groups beyound an introduction. volume 140 of Progress in Mathemtics, chapter VI. Birkhäuser, Boston-Basel-Berlin, 1996.

[KP03] I. Kim and J. R. Parker. Geometry of quaternionic hyperbolic manifolds. Math. Proc. Camb. Phil. Soc., 135:291-320, 2003.

[Loo69] O. Loos. Symmetric spaces II. Mathematics Lecture Notes Series. W.A. Benjamin, INC, New York-Amsterdam, 1969.

[Mos73] G.D. Mostow. Strong rigidity of locally symmetric spaces. volume 78 of Annals of Mathematics Studies. Oxford University Press, Princeton, New Jersey, 1973.

[Mos80] G.D. Mostow. On a remarkable class of polyhedra in complex hyperbolic space. Pac. J. Math., 86:171-276, 1980.

[PT88] R.S. Palais and Ch.-L. Terng. Critical point theory and submanifold theory. volume 1353 of Lecture Notes in Mathemtics, chapter 5. Springer-Verlag, BerlinHeidelberg-New York-London-Paris-Tokyo, 1988.

[PT99] F Podestà and G. Thorbergsson. Polar actions on rank-one symmetric spaces. J. Differential Geom., 53:131-175, 1999.

[Rud64] W. Rudin. Principles of de mathematical analysis, chapter 9. Mc Grau-Hill Book Company, second edition, 1964.

[Ste99] N. Steenrod. The topology of fibre bundles. Princeton Landmarks in Mathematics. Princeton Univercity Press, Princeton, New Jersey, 1999.

[Tom87] P. Tomter. The spherical Bernstein problem in even dimensions and related problems. Acta Math., 158:187-212, 1987.

[Tom88] P. Tomter. Existence and uniqueness for a class of cauchy problems with characteristic initial manifolds. Journal of Differential Equations, 71:1-9, 1988. 
[Tom90] P. Tomter. On dynamical systems and the minimal surface equation. In From Topology to Computation: Proceedings of the Smalefest, Berkeley, CA, pages 259269, New York, 1990. Springer.

[Tom96] P. Tomper. Minimal hypersurfaces in two-point homogeneuos spaces. Pacific Journal of Mathematics, 173(1):263-281, 1996.

[Whi78] G.W. Whitehead. Elements of homotopy theory. volume 61 of Graduate Text in Mathematics, chapter Appendix A. Springer-Verlag, New York-Heidelberg-Berlin, 1978.

[Yok68] I. Yokota. Exceptional lie group $F_{4}$ and it representation rings. Jour. Fac. Sci., Shinshu Univ., 3:35-60, 1968. 